\title{
LA- -12196
}

DE92 000694

MCNP: Photon Benchmark Problems

Daniel J. Whalen

David E. Hollowell

John S. Hendricks

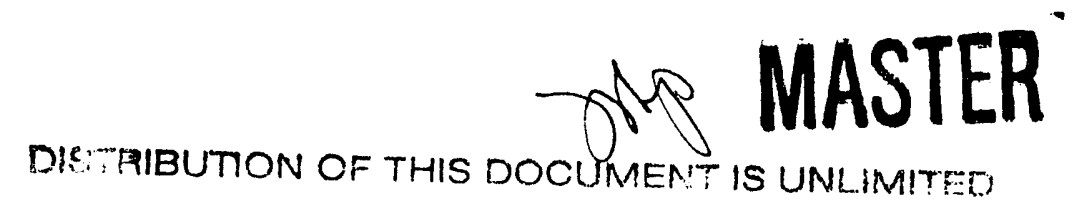




\title{
MCNP: PHOTON BENCHMARK PROBLEMS
}

by

\author{
Daniel J. Whalen \\ David E. Hollowell \\ John S. Hendricks
}

\begin{abstract}
The recent widespread, markedly increased use of radiation transport codes has produced greater user and institutional demand for assurance that such codes give correct results. Responding to these pressing requirements for code validation, the general purpose Monte Carlo transport code MCNP has been tested on six different photon problem families. MCNP was used to simulate these six sets numerically. Results for each were compared to the set's analytical or experimental data. MCNP successfully predicted the analytical or experimental results of all six families within the statistical uncertainty inherent in the Monte Carlo method. From this we conclude that MCNP can accurately model a broad spectrum of photon transport problems.
\end{abstract}

\section{INTRODUCTION}

A. Importance and Uses of Benchmarks

This report presents a series of six MCNP analytical and photon benchmark family calculations containing a total of sixteen different problems that were calculated using MCNP version 4 on the Cray Y-MP computer at Los Alamos National Laboratory. $\mathrm{MCNP}^{1}$ is a general purpose Monte Carlo radiation transport code that

1 Judith F. Briesmeister, Editor, "MCNP - A General Monte Carlo Code for Neutron and Photon Transport, Version 3A," Los Alamos National Laboratory Report LA-7396-M, Rev. 2 (1986), p. iii. 
can numerically simulate neutron, photon, and electron transport. It can solve 3$\mathrm{D}$, time-dependent, continuous energy radiation transport problems and has been adapted to operate in many different computer systems and environments.

The benchmarking of radiation transport modeling codes has become increasingly important, in part because the widespread use of such codes has proliferated dramatically in recent years. Increasing experimental costs and decreasing computational costs are making numerical transport simulation more attractive, especially when experiments might otherwise have to be conducted in hazardous environments. Also, improved computational techniques in these codes as well as faster and better computers make reliance upon numerical modeling more feasible.

Accompanying the widespread increased use of radiation transport codes is a greater demand from the user community for assurance that the codes are accurate for as broad a spectrum of problems as possible. In addition, regulatory agencies such as the DOE are insisting upon better code validation. Code quality control may even become a legal issue. These user and institutional demands motivared the calculation and compilation of the photon benchmarks presented here. Neutron benchmarks will soon follow.

Benchmarks are standard problems for which either analytical solutions or accurate experimental data exist. The transport code numerical models of such problems are of great value to code validation for the following reasons:

- they verify that the code functions properly

- they verify that the cross-section data used by the code are accurate

- they help certify that a user has learned to use the code correctly

Successful transport-problem numerical modeling resis upon two foundations: (a) validation of the code and its data, and ( $b$ ) validation of the user.

Benchmark problems constitute a standard against which the performance and accuracy of a code can be gauged. If a code can correctly predict the experimental or analytical results of a wide range of benchmarks within experimental error and Monte Carlo statistical limits, the user can generally be assured that the code functions properly. However, if errors are present in a code, they can assume many different forms, ranging from mistakes in the coding to inadequate physics treatment within the code. Such mistakes generally will cause system errors, crashes, or large errors in computed results. In the cases where the code error produces inaccurate 
results, comparison to a benchmark will detect the error. Benchmarks are also useful for checking a code's operation after it has been moved between different computers or operating systems.

To simulate the physical processes in radiation transport, MCNP uses experimentally measured cross-section data. Within the cross-section data libraries that MCNP stores and accesses, there are literally millions of numbers. Since these cross sections are experimentally determined, there are experimental uncertainties associated with them. Probably the main limitation of the ability of present transport codes to model certain problems accurately is the lack of precisely known cross sections. Benchmarks can provide very sensitive checks of uncertainties or errors in cross-section data. For example, if only a $5 \%$ error is assumed in a total cross section (a small experimental error for much of the higher energy neutron data), a $65 \%$ error $^{2}$ in the uncollided flux can accrue after only 10 mean free paths (MFP). Comparison of such results to a benchmark will demonstrate this error.

Benchmarks can also gauge a user's ability to operate a code. Learning to use powerful codes correctly can require considerable time and effort. Accompanying the power and versatility of such codes comes a greater potential for incorrect use and inaccuracy. Although the correct modeling of benchmarks cannot certify that a user has acquired total competence with a code, these models can considerably improve a user's ability and confidence.

In this report MCNP input files are provided in the Appendix as part of the description of each benchmark. If users plan to study these benchmarks to gain competence with MCNP, we strongly urge that they first attempt to set up the problems by themselves before studying the input files. This effort will help ensure that users gain personal ability with the code rather than a simple understanding of what someone else has done. Each problem is described in sufficient detail for users to correctly set up the problem geometry, source, and tally set. Also, insight is provided into how variance reduction techniques were applied for each problem.

\section{B. Benchmark Guidelines}

The main purpose for benchmarking MCNP is to establish that it functions properly and can model a wide range of problems accurately. Besides whether or

2 Thomas P. Wilcox, Jr. and Edward M. Lent, "COG - A Particle Transport Code Designed to Solve the Boltzmann Equation for Deep Penetration (Shielding) Problems," Vol. 4, "Benchmark Problems," Lawrence Livermore National Laboratory Report M-221-4 (12/2/88), p. 2. 
not a code functions properly, the two central questions of both new and experienced transport code users are as follows:

- what are the strengths and limitations of a given transport code, and what kinds of problems does it not solve well?

- when can one be certain that transport code results are correct, and how much confidence can be placed in them?

It is important to address these questions and to see how benchmarks can answer them, at least in part.

One of MCNP's strengths is that it can model problems ranging from reactor design to radiation shielding to medical physics. The MCNP code has undergone over 300 person years of development and refinement, and has been successfully used worldwide at hundreds of installations. ${ }^{3}$ These attributes make the possibility of significant errors in the code very remote. The benchmark problems reported here provide additional excellent confirmation of how well MCNP models a wide range of problems. However, they cannot guarantee that MCNP can accurately simulate every conceivable problem.

The main limitation in MCNP's ability to model problems correctly is the lack of well known cross sections. It is impossible to list what problems MCNP can or cannot adequately simulate. Nevertheless, a good general guideline is that MCNP can model well those problems whose cross sections have been experimentally well measured. Special care should be exercised in the interpretation and use of MCNP results for problems whose cross sections are not well characterized. An example of such a problem is one involving very high energy neutrons whose scattering and absorption cross sections are poorly known.

The emphases in indistrial and scientific research tend to define what cross sections are well known and consequently what problems can be simulated well. Future shifts in research foci will fill in the gaps in cross-section data that restrict present code performance. The capabilities of MCNP are constantly being upgraded as new data sets and computational techniques become available. MCNP's abilities and accuracy will continue to be sharpened as future problems are modeled and analyzed.

The question of whether code results are correct and what confidence can be placed in them is a challenging one. The only certain way researchers can know

3 Briesmeister (Ref. 1), p. iv. 
their code results are valid is to obtain experimental or analytical results for a code comparison. Otherwise, there exists no single standard or algorithm that enables code users to determine how much confidence they can place in their code estimates. Nevertheless, transport codes can be used to predict experimental results or guide experimental design without knowing the results beforehand if users take the following steps.

First, when modeling any problem with a transport code, it is important, if possible

- to understand the problem or experiment and its physics well enough to have at least a general idea of what its results will be

- to understand the code's function, physics, and data library well enough not to use it as a "black box."

This knowledge will provide researchers with some ability to judge code predictions and know beforehand whether the code physics adequately treats the problem. The blind use of any transport code is an unsound practice and usually leaves researchers with little more meaningful information or insight than when they began. Next, code results should always be checked for internal consistency (i.e., do tally results confirm one another?) This checking will catch many types of internal code errors, if they exist.

It may happen that even the general results of a problem or planned experiment cannot be predicted before the fact, or that a researcher is not certain a code is validated for a class of problems. In this situation, users can validate their code for their experiment as follows:

- find an already performed experiment that is as similar as possible to the one in question

- use the code to model its measurements and data

- compare the code estimates to the measured data

This technique has several advantages. First, such a benchmark can be a "dress rehearsal" for the experiment being investigated. Second, if the code models the similar experiment well, the code user can generally be assured that the new experiment will also be successfully modeled. Third, the benchmark may give an experimenter further insight into the physics of the new experiment. Therefore, 
even if experimenters do not know a priori whether their code will give valid results or how some planned experimental data should appear, it is possible to establish what degree of confidence can be placed in the code.

\section{Problem Overview}

The six benchmark problems chosen for presentation here involve photon transport only. They are identical to those used by Thomas Wilcox and Edward Lent at LLNL to validate their COG Monte Carlo code. ${ }^{4}$ Three of the benchmarks have analytical solutions and were taken to be photon problems. The other three were. the focus of actual experimental study. Because these benchmarks were also used to validate $\mathrm{COG}$, the MCNP results of each problem were compared to the corresponding COG results as well as the analytic or experimental results. An overview of these benchmarks appears in Tables 1 and $2{ }^{4}$

The analytical problems were chosen for study in part because they require modified physics treatments which test MCNP's flexibility. They were also chosen because their precise results allow the detection of small computational errors that might otherwise be masked by experimental error. Analytical problems are also free of the ambiguities in experimental description that are sometimes present in scientific papers. Such ambiguities can greatly complicate the numerical simulation of an experimental benchmark.

The experimental problems were chosen for simulation because they test MCNP's ability to solve more complicated problems. These experiments involve deep penetration and scattering which heavily test MCNP's variance reduction capabilities. They also cover a wide range of photon energies and material compositions. There are also large differences in experiment geometry among the three benchmarks. These problems demonstrate the broad spectrum of experimental conditions for which MCNP is validated.

\footnotetext{
4 Wilcox and Lent (Ref. 8), pp. 4-9.
} 
TABLE 1

DESCRIPTION OF MCNP PHOTON BENCHMARK PROBLEMS

\begin{tabular}{|c|c|c|c|c|}
\hline $\begin{array}{l}\text { Problem } \\
\text { Number } \\
\end{array}$ & $\begin{array}{l}\text { Problem } \\
\text { Type }\end{array}$ & $\begin{array}{c}\text { Sub- } \\
\text { Category }\end{array}$ & Description & $\begin{array}{l}\text { Energy } \\
\text { Range }\end{array}$ \\
\hline \multirow[t]{4}{*}{1} & Analytic & & $\begin{array}{l}\text { Point source in an } \\
\text { infinite medium with } \\
\text { constant isotropic-scatter/ } \\
\text { absorption cross sections: }\end{array}$ & \\
\hline & & $\mathrm{a}$ & $\sigma_{s c a t}=0, \sigma_{a b s}=\sigma_{t o l}$ & \\
\hline & & $\mathrm{b}$ & $\sigma_{s c a t}=0.3 \sigma_{t o t}, \sigma_{a b s}=0.7 \sigma_{t o t}$ & $1 \mathrm{MeV}-1 \mathrm{KeV}$ \\
\hline & & $\mathrm{c}$ & $\sigma_{s c a t}=0.9 \sigma_{t o t}, \sigma_{a b s}=0.1 \sigma_{t o t}$ & \\
\hline \multirow[t]{2}{*}{2} & Analytic & & $\begin{array}{l}\text { Point source centered } \\
\text { in a spherical scatterer }\end{array}$ & \\
\hline & & & $\begin{array}{l}\text { with constant isotropic- } \\
\text { scatter and absorption } \\
\text { cross sections }\end{array}$ & $1 \mathrm{MeV}-1 \mathrm{KeV}$ \\
\hline \multirow[t]{4}{*}{3} & Analytic & $\mathrm{a}$ & Point source in an infinite & Al: $1 \mathrm{MeV}-1 \mathrm{KeV}$ \\
\hline & & $\mathrm{b}$ & medium with Compton & $\mathrm{Al}: 10 \mathrm{MeV}-1 \mathrm{KeV}$ \\
\hline & & c & scattering, pair production, & $\mathrm{Pb}: \quad 1 \mathrm{MeV}-1 \mathrm{KeV}$ \\
\hline & & $\mathrm{d}$ & and photo-electric effects only & $\mathrm{Pb}: 10 \mathrm{MeV}-1 \mathrm{KeV}$ \\
\hline 4 & $\begin{array}{l}\text { Experimental/ } \\
\text { Computational }\end{array}$ & & $\begin{array}{l}\text { Uniform }{ }^{60} \text { Co surface } \\
\text { source on an infinite air- } \\
\text { ground interface. }\end{array}$ & $1.33 \mathrm{MeV}-1 \mathrm{KeV}$ \\
\hline 5 & Experiment & & $\begin{array}{l}\text { A cone } \gamma \text {-source is directed } \\
\text { skyward and skyshine doses } \\
\text { on the ground are measured }\end{array}$ & $1.33 \mathrm{MeV}-1 \mathrm{KeV}$ \\
\hline \multirow[t]{7}{*}{6} & Experiment & & $\begin{array}{l}\text { Cylindrical } \gamma \text {-ray spectrometer } \\
\text { with six point-source energies: }\end{array}$ & \\
\hline & & $\mathrm{a}$ & ${ }^{60}$ Co source: $1.33 / 1.17 \mathrm{MeV}$ & \\
\hline & & $\mathrm{b}$ & ${ }^{137} \mathrm{Cs}$ source: $661 \mathrm{KeV}$ & \\
\hline & & $\mathrm{c}$ & 198 Au source: $412 \mathrm{KeV}$ & Source Energy- \\
\hline & & $\mathrm{d}$ & ${ }^{170} \mathrm{Tm}$ source: $84 \mathrm{KeV}$ & $1 \mathrm{KeV}$ \\
\hline & & e & ${ }^{241}$ Am source: $59.6 \mathrm{KeV}$ & \\
\hline & & $\mathrm{f}$ & Sm K $\alpha$ source: $39.9 \mathrm{Kev}$ & \\
\hline
\end{tabular}


TABLE 2

DESCRIPTION OF MCNP PHOTON BENCHMARK PROBLEMS

\begin{tabular}{|c|c|c|c|}
\hline $\begin{array}{l}\text { Problem } \\
\text { Number } \\
\end{array}$ & $\begin{array}{l}\text { Quantities } \\
\text { Computed } \\
\end{array}$ & $\begin{array}{c}\text { Principal Material } \\
\text { Composition }\end{array}$ & Comments \\
\hline 1 & $\begin{array}{l}\text { Particle current } \\
\text { through a surface }\end{array}$ & $\begin{array}{l}\text { Arbitrary (the photon } \\
\text { physics depended only } \\
\text { on } \sigma_{\text {tot }} \text { and } \rho \text {, which } \\
\text { were arbitrary) }\end{array}$ & $\begin{array}{l}\text { Required minor MCNP } \\
\text { code alteration to restrict } \\
\text { the photon physics and to } \\
\text { accept constant cross sections }\end{array}$ \\
\hline 2 & $\begin{array}{l}\text { Flux at a point } \\
\text { outside a sphere }\end{array}$ & $\begin{array}{l}\text { Arbitrary : problem } \\
\text { physics was the same } \\
\text { as for benchmark } 1\end{array}$ & $\begin{array}{l}\text { Requires MCNP source } \\
\text { code alterations as in } \\
\text { benchmark } 1 .\end{array}$ \\
\hline 3 & $\begin{array}{l}\text { Energy response/MeV: } \\
\text { (energy flux over a } \\
\text { sphere) } x 4 \pi \mathrm{r}^{2} \mathrm{e}^{\mu r} / \mathrm{MeV} \text {, } \\
\text { where } \mu \mathrm{r}=\# \text { of } \mathrm{MFP} \\
\text { of the sphere radius }\end{array}$ & $\begin{array}{l}\text { Aluminum } \\
(1 \& 10 \mathrm{MeV}) \\
\text { Lead } \\
(1 \& 10 \mathrm{MeV})\end{array}$ & $\begin{array}{l}\text { Photon electron } \\
\text { physics restricted to } \\
\text { Compton scattering, pair } \\
\text { production, and photo- } \\
\text { electric capture }\end{array}$ \\
\hline 4 & $\begin{array}{l}\text { Dose buildup factor } \\
\text { (total dose/uncollided } \\
\text { dose) } 3 \mathrm{ft} \text {. above } \\
\text { ground. Angular kerma } \\
\text { rate ([ergs deposited in } \\
\text { the material]/grams } \cdot \mathrm{sec} \text {. } \\
\text { steradian) around a point } \\
3 \mathrm{ft} \text {. above ground. }\end{array}$ & $\begin{array}{l}\text { Air and } \\
\text { soil }\end{array}$ & $\begin{array}{l}\text { Deep penetration and } \\
\text { scattering problem: a } \\
\text { difficult variance } \\
\text { reduction challenge }\end{array}$ \\
\hline 5 & $\begin{array}{l}\mu \mathrm{REM} / \mathrm{hr} / \mathrm{Curie} \text { at } \\
\text { outdoor ground level } \\
\text { detectors }\end{array}$ & $\begin{array}{l}\text { Air and } \\
\text { soil }\end{array}$ & $\begin{array}{l}\text { Scattering problem - } \\
\text { experimental description } \\
\text { ambiguities were present }\end{array}$ \\
\hline 6 & $\begin{array}{l}\text { Dose ratios of TLDs } \\
\text { in a teflon cylinder }\end{array}$ & $\begin{array}{l}\text { Air, iron, } \\
\text { teflon }\end{array}$ & $\begin{array}{l}\text { High scattering } \\
\text { problem, especially at } \\
\text { lower source energies }\end{array}$ \\
\hline
\end{tabular}




\section{BENCHMARK PROBLEM ONE - INFINITE MEDIUM PROB- LEM WITH A CONSTANT CROSS SECTION AND ISOTROPIC SCATTERING}

\section{A. Problem History and Description}

The isotropic point source in a homogeneous infinite medium is a classic example of an early particle transport problem with an analytical solution, and was studied by Case et al. in 1953. In this problem, a point source of particles is located in a homogeneous infinite medium where either absorption or isotropic scattering occurs, each with a cross section that is constant for all particle energies (see Fig. 1.1). The number of pariicles at several given distances from the point source is then computed. The analytical solution to this problem along with its numerical results is discussed in detail in Case et al. ${ }^{5}$

MCNP was used to calculate the results of this problem (which was taken to be a photon problem) for three distinct cases:

1. $\sigma_{a b s}=\sigma_{t o t a l}, \sigma_{s c a t}=0$ - in which case the number of photons surviving to a distance $\mathrm{r}$ from the source is $\mathrm{e}^{-\mu r}$, where $\mu$ is the inverse of the photon mean free path length (MFP).

2. $\sigma_{a b s}=0.7 \sigma_{\text {total }}, \sigma_{\text {scat }}=0.3 \sigma_{\text {total }}$

3. $\sigma_{a b s}=0.1 \sigma_{\text {total }}, \sigma_{\text {scat }}=0.9 \sigma_{\text {total }}$

The MCNP results of these calculations for $30 \%$ and $90 \%$ scattering were compared to the analytical results for the problem in Tables 17 and 18 of Case et al. ${ }^{6}$ along with the corresponding COG results. ${ }^{7}$ Since the total photon cross section is constant for all particle energies (and was taken to be the same in all three cases), the photon MFP was also constant for all energies. This problem was chosen as a benchmark in part because it has an analytical solution that allows any potential small MCNP computational errors to be readily detected. It was also chosen to test MCNP's ability to selectively alter, include, or ignore different elements of photon/electron physics. Furthermore, the problem is also conveniently independent of real cross sections and their uncertainties.

5 K. N. Case, F. de Hoffmann, and G. Placzek, Introduction to the Theory of Neutron Diffusion, Vol. 1, U.S. Government Printing Office, Washington, D.C. (1953), pp. 66-101.

6 Ibid, pp. 100-101.

7 Wilcox and Lent (Ref. 8), pp. 12-13. 


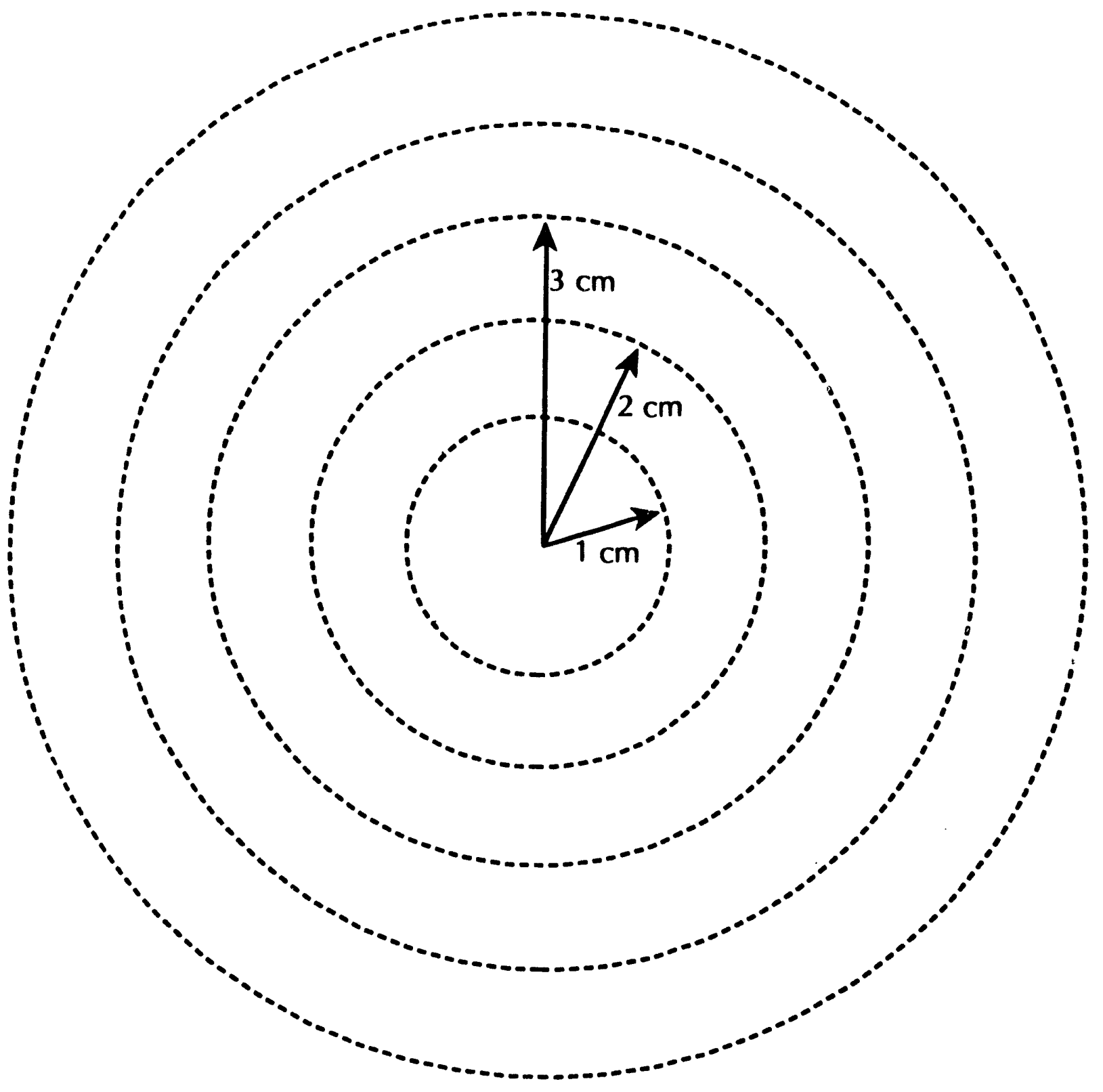

Fig. 1.1. Geometry of the point source in an infinite medium. 


\section{B. MCNP Problem Model}

1. MCNP Geometry. A point isotropic source of gamma rays (with an arbitrarily chosen energy of $1.0 \mathrm{MeV}$, since the $\sigma$ 's are consiant) was placed at the origin of a coordinate system. A spherical cell with a .3 MFP radius ( 1 photon MFP was $1 \mathrm{~cm}$ in all three cases) was then centered at the origin. Fourteen additional cells, each defined to be the region between two concentric spheres centered at the origin, were then placed around the sphere cell. The first five concentric shell cells were $0.2,0.3,0.2,0.5$, and $0.5 \mathrm{MFP}$ thick, respectively. The next eight shell cells were each 1 MFP thick, and the outermost cell was 15 MFP thick. The problem boundary was the outermost sphere (of 25 MFP radius), and the region beyond it was made a void.

The input file for the $30 \%$ scattering case is found in Table A.1 of the Appendix.

2. Cross-Sections Material Composition. The particle mean free pathlength was chosen to be one $\mathrm{cm}$ when the problem was originally solved analytically. ${ }^{8}$ The corresponding MCNP photon MFP is found from the total microscopic photon cross section and the atom density of the medium as follows:

$1 \operatorname{MFP}($ in $\mathrm{cm})=\left\{\left[\sigma_{\text {tot }}\right.\right.$ (in barns $\left.)\right] \times\left[\rho\left(\right.\right.$ atom density, in $\left.\left.\left.10^{24} \frac{\text { atoms }}{\mathrm{cm}^{3}}\right)\right]\right\}^{-1}$

In the problem model, $\sigma_{t o t}$ and $\rho$ were arbitrarily chosen to be constants that satisfy the criterion that $1 / \sigma \rho=1 \mathrm{~cm}$, and were kept constant for all three cases. The medium was arbitrarily chosen to be hydrogen, since the physics of this problem depends only on $\sigma$ and $\rho$, not on what material the medium actually is.

3. MCNP Photon/Electron Physics. Next, it was necessary to modify MCNP to enable it to do two things:

1. accept user-input absorption and isotropic scatter cross sections constant for all energies.

2. cause photons to undergo either total absorption or isotropic scattering only lic other photon/electron physics would occur.

This modification was accomplished by minor alteration of MCNP, shown in the patch file listing in Table A.2 of the Appendix. The code alterations to MCNP instructed it to treat a photon absorption as a photoelectric capture and to model an isotropic scatter as a photoelectric fluorescence. In this scattering model, a photon interacts with an electron, ejects it from the atom, and is annihilated in the process.

8 Case et al. (Ref. 2), p. 100. 
The elertron is then reabsorbed back irto an atom with a photon consequently being isotropicaily emitted. The net result of this process is an isutropic photon scatter. The code alterations to MCNP assigned a constant cross section to each process that is specified by the user in the input file by an RDUM card of the form

\section{RDUM $\sigma_{\text {tot }} \sigma_{a b s}$ (in barns)}

4. Code Tallies. After the code was modified, surface photon flux (F2:P) tallies were placed on the first 14 spheres used to specify the cell geometry (the outermost sphere was not tallied for particles). The results of each tally (in particles $/ \mathrm{cm}^{2}$ ) were multiplied by the area of the tally sphere using the AREA card:

\section{AREA $7854 \quad 1$ 13R}

The first number is the surface area of the $25 \mathrm{~cm}$ sphere. The fourteen ones that follow cause the tally to be divided by one rather than the area. Thus, the effect is to multiply the result of each tally by its sphere surface area. Multiplying each flux estimate by the area of its tally surface yields the number of photons present at each tally surface.

5. Variance Reduction. With the tallies arranged, importances were next assigned to each cell. The importance of each cell was initially chosen to increase by a factor of two for every MFP between the origin and the inner surface of the cell. They were then adjusted by trial and error to equalize the particle population (and therefore optimize sampling) in each cell. The three input files were then run to obtain the MCNP results for the problem.

\section{Results and Discussion}

1. The Three Cases. The MCNP data for pure absorption, $30 \%$ scattering, and $90 \%$ scattering appear in Figs. 1.2, 1.3, and 1.4, respectively, along with the analytical results. The COG results also appear in the $30 \%$ and $90 \%$ scattering cases in Figs. 1.3 and 1.4. The number of particles found at a given distance $r$ from the source (normalized per source particle) is plotted as a function of this source distance. In the pure absorption case, the MCNP results are within one standard deviation of the analytical results in $86 \%$ of the data. In the other two cases, MCNP is within one standard deviation of the analytical data $67 \%$ of the time. 


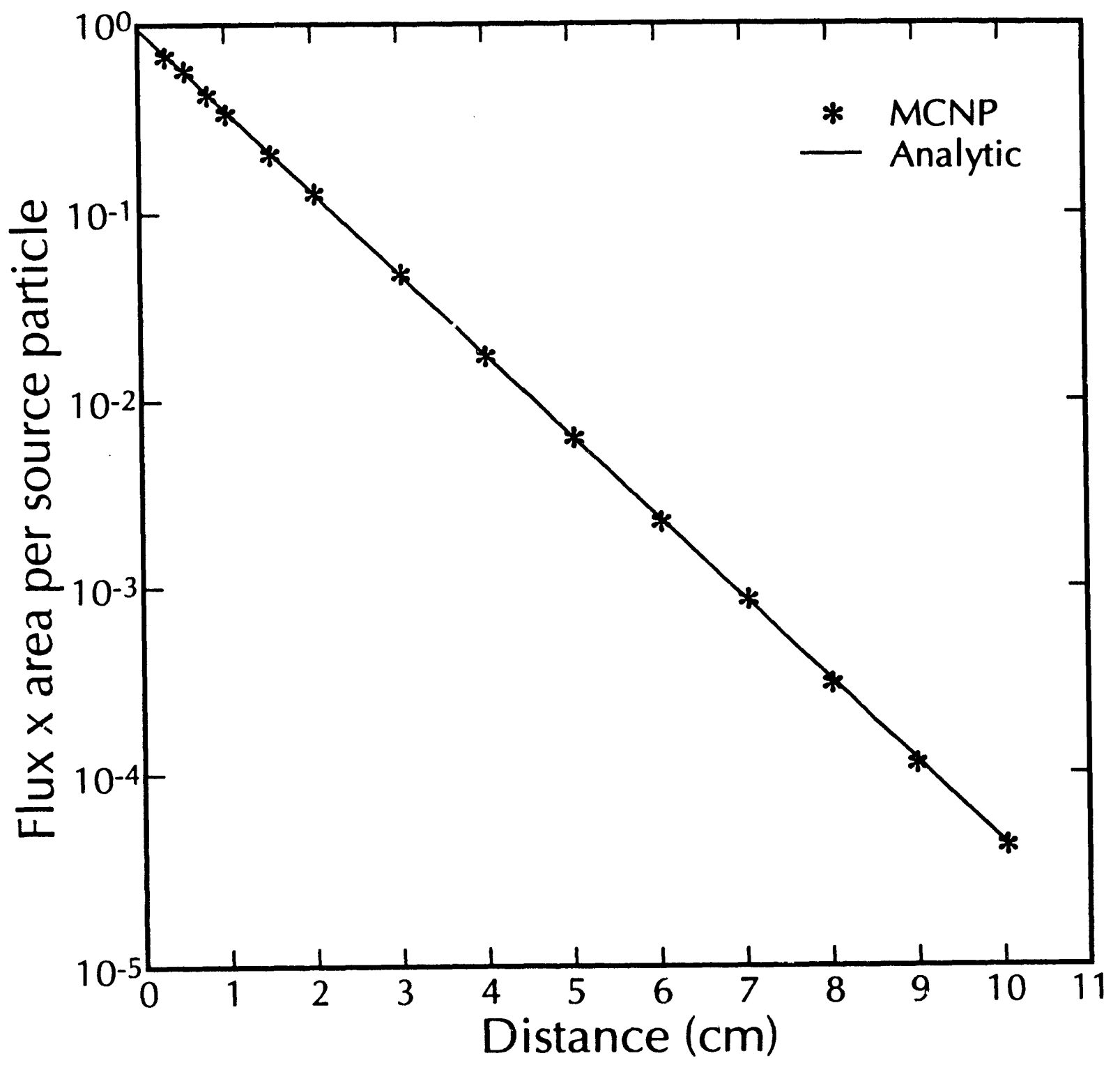

Fig. 1.2. Particle current as a function of distance: $0 \%$ scattering. 


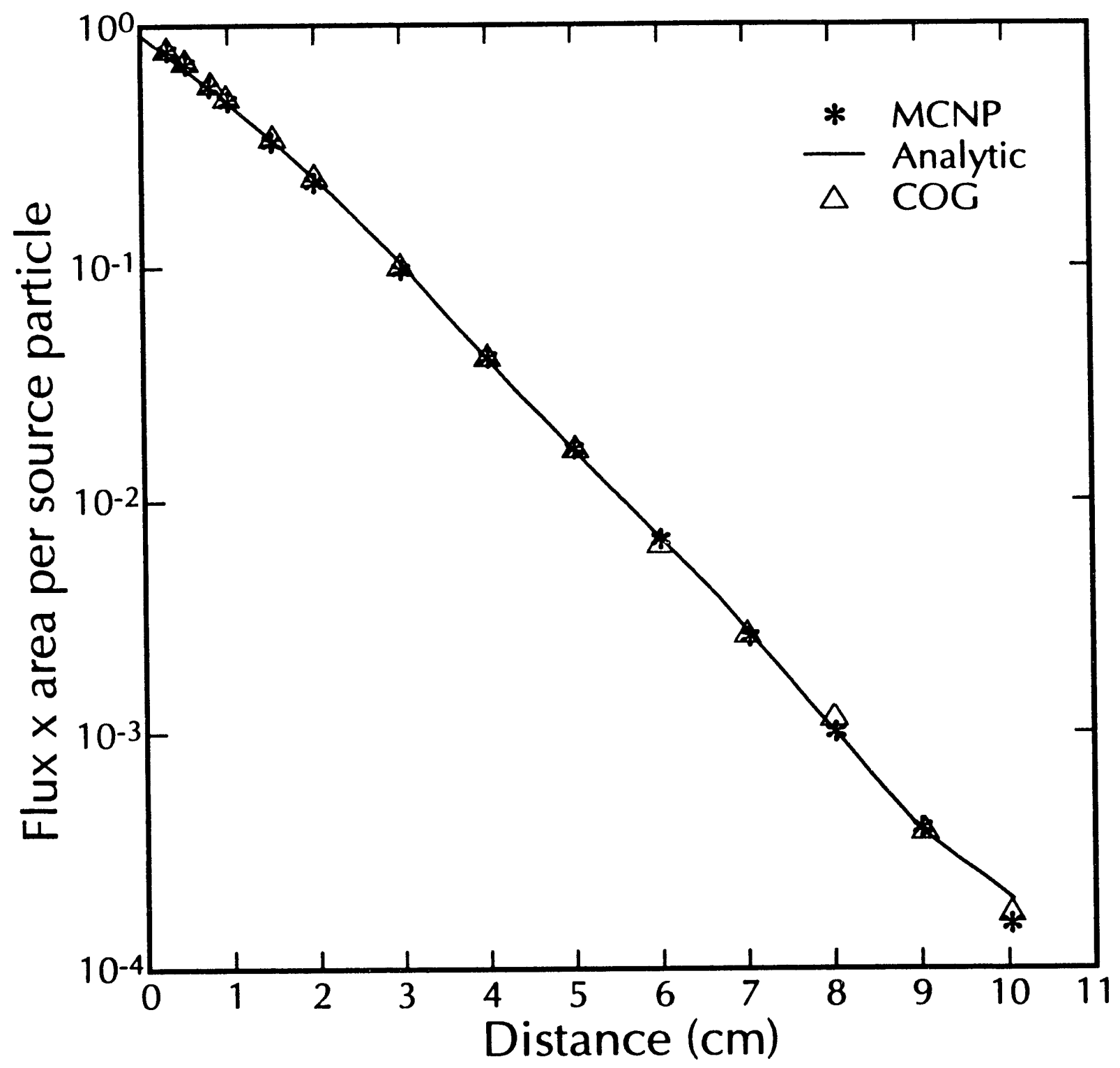

Fig. 1.3. Particle current as a function of distance: $30 \%$ scattering. 


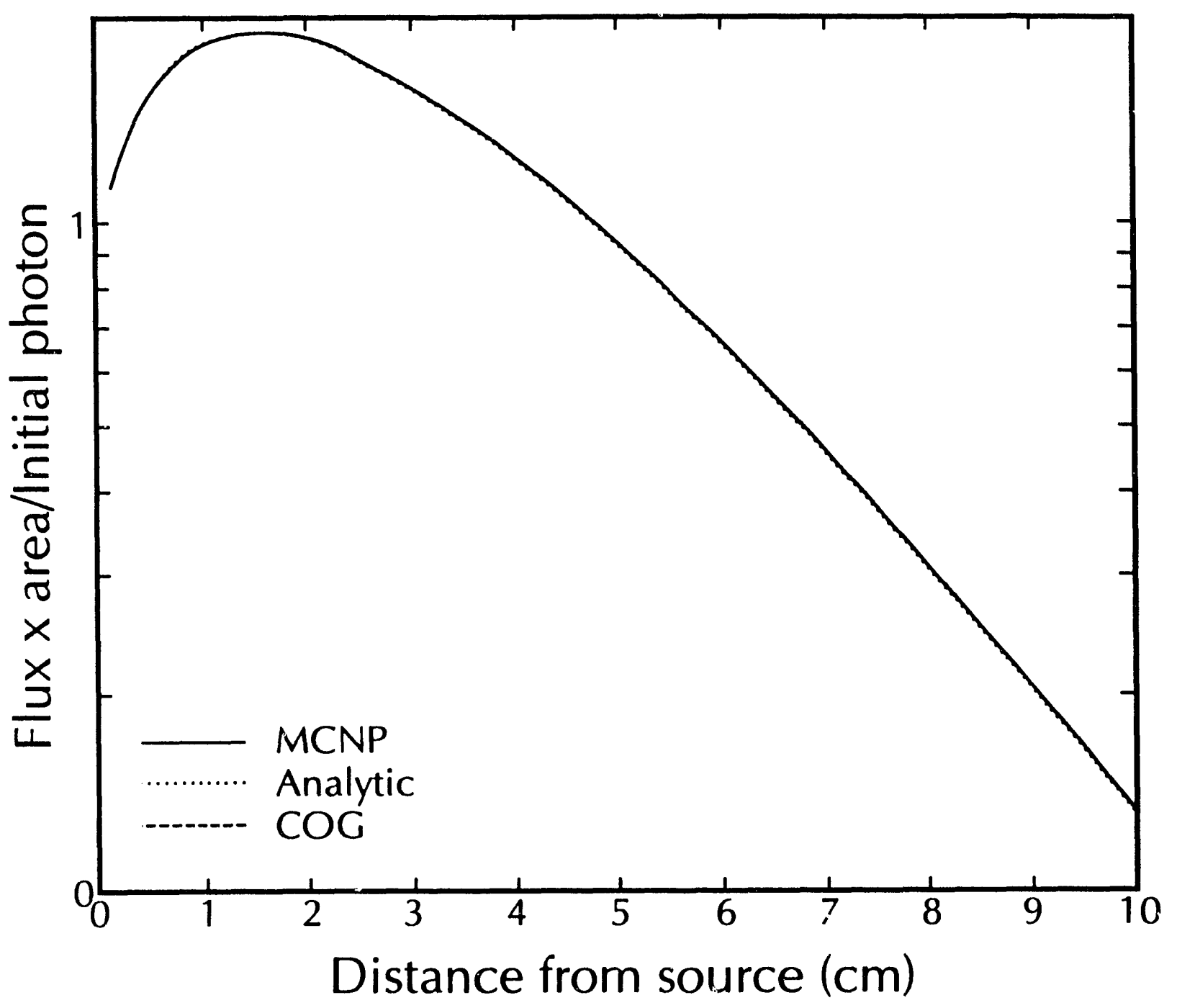

Fig. 1.4. Particle current as a function of distance: $90 \%$ scattering. 
Figure 1.2 demonstrates that, for the pure absorption case, the number of particles a distance $r$ from the source indeed decreases by $\mathrm{e}^{-r}$. In the $90 \%$ scattering case, a maximum can be seen in the data at $\mathrm{r} \simeq 1.6 \mathrm{~cm}$ before exponential dropoff occurs. This peak is present because enough scattering occurs in the problem to backscatter photons to (and therefore maximize their numbers at) this distance. These data demonstrate that MCNP accurately models this problem within Monte Carlo statistical limits.

2. Statistical Interpretation. In the pure absorption case, MCNP is within one standard deviation of the analytical data in $86 \%$ of the points. It might initially be thought that such statistical agreement should be seen in only $67 \%$ of the data. However, this agreement is true only if the estimate of each tally is independent of the estimates of the other tallies. The estimates of the tallies in this problem are correlated because tallies on the outer surfaces are from the same particles tallied crossing the inner surfaces.

Had each problem been run ton times, each run beginning with a different random number seed and a non-overlapping number sequence, the tally estimates of each run would be statistically independent of those in other runs. The tally estimate at a given distance in one run would be statistically uncorrelated with the tally estimates at the same distance in the other nine runs. It would then be expected that for this given distance, $67 \%$ of the estimates of the ten runs would be within one standard deviation of the analytical result for that distance.

Benchmark two was run ten times to verify that this result happens. The tallies of that problem are more strongly correlated than those in this benchmark. In benchmark two, every particle contributing to the first tally survived to score at all the other tallies. It was found that for a given distance, $67 \%$ of the problem run results were within one standard deviation of the analytical data for that distance. 


\section{BENCHMARK PROBLEM TWO - SIMPLE SPHERICAL SCAT- TERER}

\section{A. Problem History and Description}

This simple spherical scatterer problem was devised by Edward Lent and Thomas Wilcox at LLNL to test the point detector tallies in the COG code. ${ }^{9}$ In this analytical problem, an isotropic point source of particles is located at the center of a $1-\mathrm{cm}$ sphere surrounded by vacuum. The sphere is composed of a homogeneous medium in which either particle absorption or isotropic scattering occurs, each with a cross section that is constant for all particle energies. The uncollided flux and firstcollided flux (due to particles undergoing only one collision) are then computed at a given distance outside the sphere. This problem was taken to be a photon problem for convenience.

Wilcox and Lent used COG to calculate the uncollided and first-collided fluxes at a point $10 \mathrm{~cm}$ from the center of the sphere. The atom density and $\sigma_{t o t}$ of the sphere medium were chosen to result in a photon mean free pathlength of one $\mathrm{cm}$ there. Because $\sigma_{\text {tot }}$ was made constant iur all photon energies, the photon MFP is also independent of energy. The scattering and absorption cross sections were set at $0.3 \sigma_{\text {tot }}$ and $0.7 \sigma_{\text {tot }}$, respectively. The uncollided flux ${ }^{10}$ at a point outside the sphere a distance $\alpha$ from its center is

$$
\Phi_{o}=\frac{e^{1}}{4 \pi \alpha^{2}}
$$

For $30 \%$ scattering, the first collided flux at a point outside the sphere is given by the integral ${ }^{11}$ (see Fig. 2.1):

$$
\Phi_{1}=0.3 \int_{-1}^{1} d \mu \int_{0}^{r} 2 \pi r^{2} d r\left[\frac{e^{-r}}{4 \pi r^{2}} \frac{e^{-t}}{4 \pi \ell^{2}}\right]
$$

where:

\footnotetext{
9 Wilcox and Lent (Ref. 8), p. 16.

10 Ibid, p. 16.

11 Ibid, p. 16.
} 


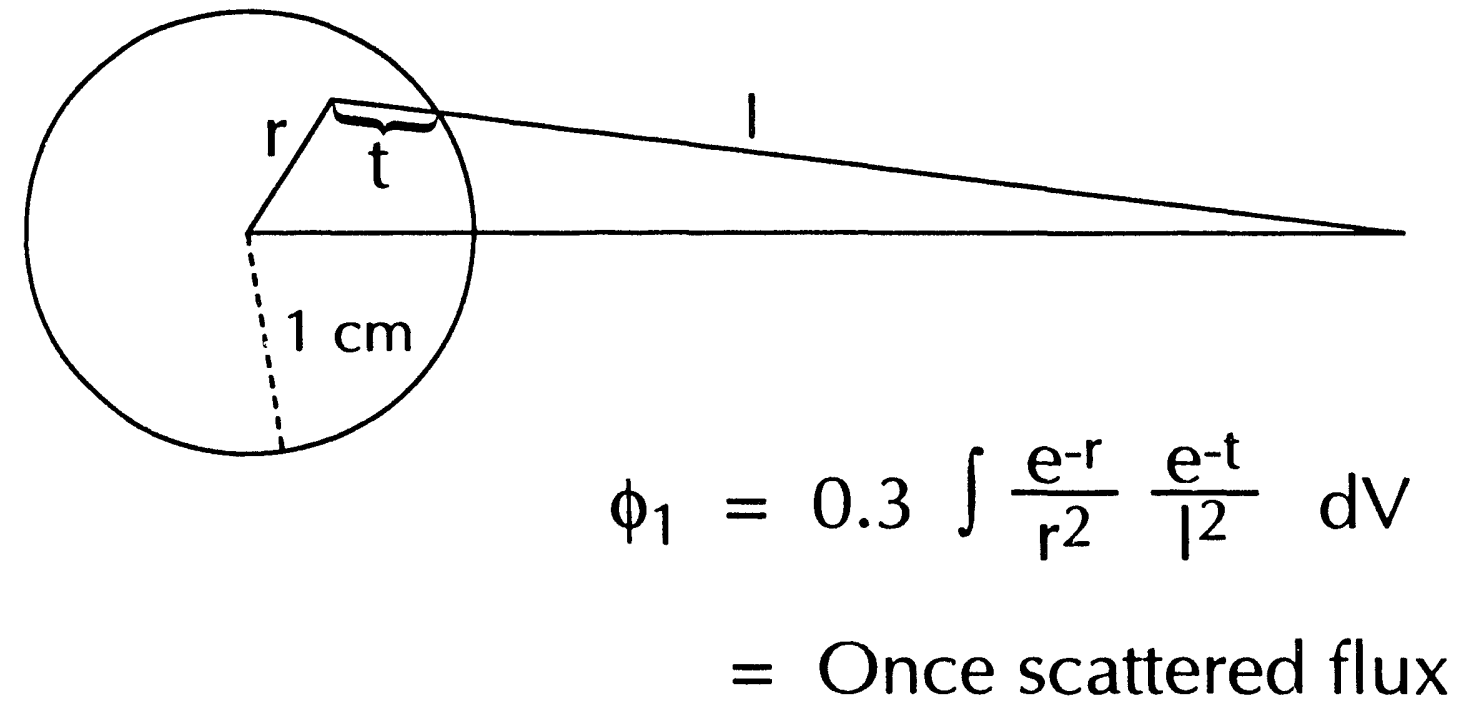

Fig. 2.1. The once-scattered flux outside a simple spherical scatterer. 
- the integral is over the spherical volume

- " $\ell$ " is the distance between the integration volume element and the point of observation outside the sphere

- " $t$ " is the distance between the integration volume element and the surface of the sphere along the line segment defined by " $\ell$ ".

Like the first benchmark, this problem was chosen for study partly because it has an analytical solution that will allow the detection of small computational errors. It is also conveniently immune to real cross-section uncertainties, and it tests MCNP's ability to count collisions. MCNP was used to compute the uncollided and firstcollided photon fluxes at ten different points outside the scattering sphere. The first-collided fluxes were then compared to the analytic results and the COG result at $\alpha=10 \mathrm{~cm}$. At each position, the integral expression for the first-collided flux had to be evaluated numerically. This evaluation was done in cylindrical coordinates, using several hundred integration zones in both radial and axial directions.

\section{B. MCNP Problem Model}

1. MCNP Geometry. A point isotropic source of gamma rays (with an arbitrarily chosen energy of $1.0 \mathrm{MeV}$, since $\sigma_{t o t}$ is independent of energy) was placed at the origin of a coordinate system. A spherical shell with a one MFP radius ( $1 \mathrm{MFP}$ was one $\mathrm{cm}$ in this problem) was centered at the origin. This cell defined the scattering sphere. Eleven additional cells, each defined to be the region between two concentric spheres centered at the origin, were then placed around the sphere cell. The first two shell cells were 0.5 MFP thick, while the next eight were 1 MFP thick. The outermost shell was 0.1 MFP thick, and the outer sphere of this cell formed the problem boundary. The shell cells were all defined to be in vacuum.

The input file for this problem is found in Table A.3 of the Appendix.

2. Cross Sections, Materials, and MCNP Physics. The sphere medium atom density, total cross section $\sigma_{\text {tot }}$, and MCNP photon/electron physics of this problem are identical to those used in benchmark one. The MCNP code itself was altered in the same way as in benchmark one to accept user-input scatter and absorption cross sections that are constant for all photon energies and to only allow photon absorption and isotropic scattering. How this was done, along with how $\sigma_{t o t}$ was calculated, is described in detail in benchmark one. 
3. Code Tallies. Once the code was modified and the cross sections were established, two sets of photon flux tallies were set up. Surface photon flux tallies were placed on the $1.5-\mathrm{cm}$ and 2- through $10-\mathrm{cm}$ spheres. Ring detectors centered around the z-axis were placed at 2, 4, 6, 8, and 10 MFP from the sphere center. The fluxes from each set of tallies were binned accordi.ng to how many collisions the photons underwent by using MCNP's FT/FU collision counter:

\section{FT INC}

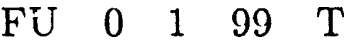

Photons that had no collisions and just one collision were individually distributed into the ' 0 ' and ' 1 ' bins above. Two sets of tallies were used so their results could be compared.

4. Variance Reduction. No variance reduction was required.

\section{Results and Discussion}

1. Results. The data for this problem are plotted in Fig. 2.2. The oncecollided flux per source photon is graphed as a function of distance from the sphere center. The analytical results (solid line evaluated at ten positions) are included with the MCNP surface tally data (long dashed line mostly hidden by the solid line) and ring detector data (short dashed line). The single COG result ${ }^{12}$ is denoted by a "C". All ten of the surface tally scores and all five ring detector estimates are within one standard deviation of the analytical results.

2. Statistical Interpretation. At first, it might be thought that such statistical agreement should only be seen in $67 \%$ of the tallies. However, this is true only if the estimate of each tally is uncorrelated with the estimates of the other tallies. The tallies in this problem are correlated because tallies on the outer surfaces are from the same particles tallied crossing the inner surfaces. The detector tallies are similarly correlated.

A way was found to obtain uncorrelated data for this problem. In ten separate runs, MCNP was used to compute the once-collided flux at a point $10 \mathrm{~cm}$ from the center of the spherical scatterer. This computation was done with a sphere surface tally. Each of the ten runs was begun with a different random number seed and a non-overlapping random number sequence. The 10-cm tally estimate

12 Ibid, p. 16. 


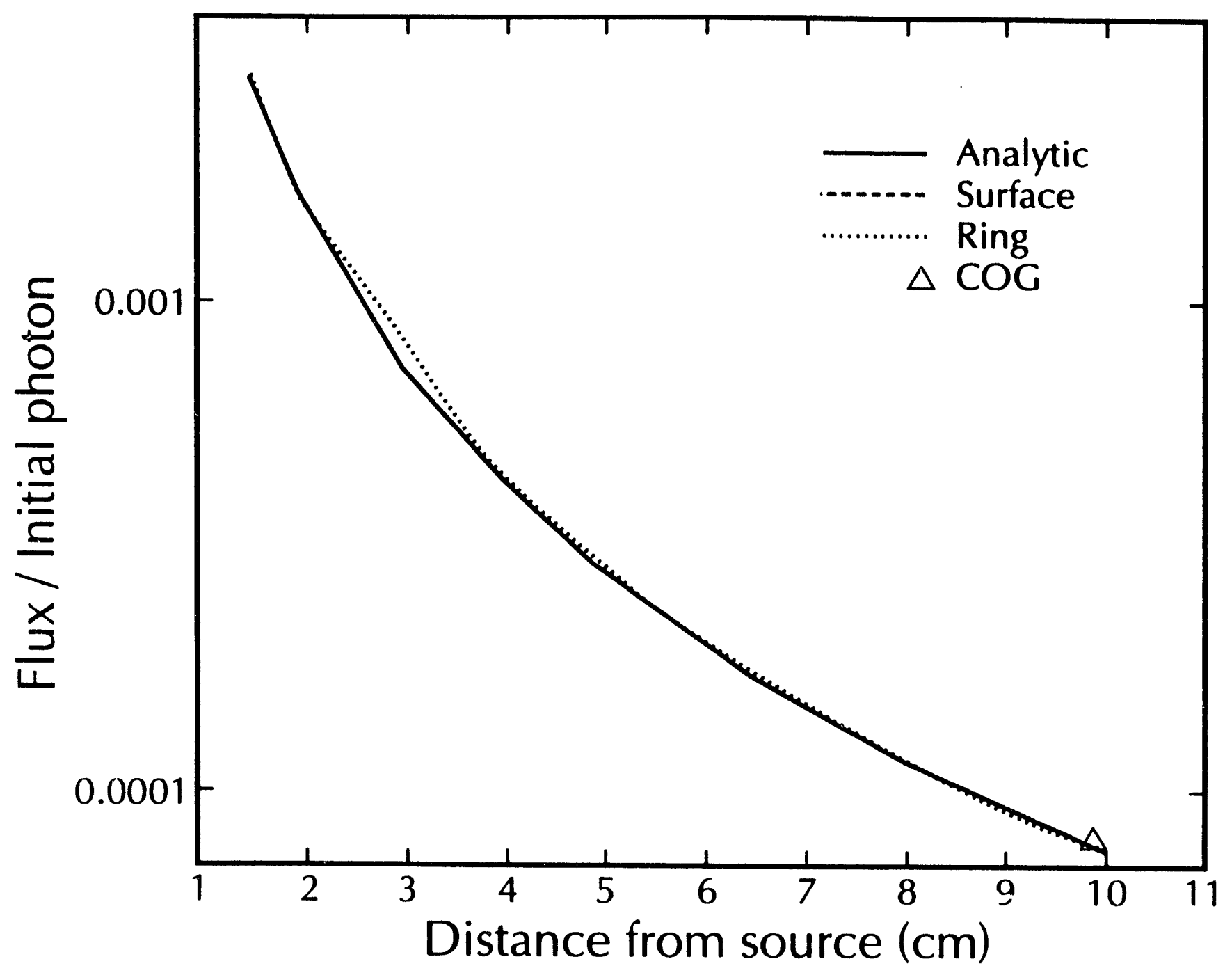

Fig. 2.2. The once-scattered flux outside the spherical scatterer. 
of each run should, therefore, be independent of the 10 -cm tiily estimates of the other runs. Seven of the ten independent tally scores were found to be within one standard deviation of the analytical result for $10 \mathrm{~cm}$. From these results, it is evident that MCNP is consistently in good agreement with the analytical solution to this problem.

\section{BENCHMARK PROBLEM THREE - POINT GAMMA RAY SOURCE IN AN INFINITE MEDIUM}

\section{A. Problem History and Description}

The point gamma source in a homogeneous infinite medium is one of a series of analytical moments method calculations of gamma ray penetration by $\mathrm{H}$. Goldstein and J. E. Wilkins done in $1954 .^{13}$ The moments method is a technique whereby the Boltzmann transport equation can be simplified and solved exactly for certain types of radiation transport problems. ${ }^{14}$ This technique allowed some early transport problems to be solved numerically with very limited computational power. Goldstein and Wilkins' calculations of gamma ray penetration are classic examples of solutions to these early transport problems.

In this problem, a point source of isotropic monoenergetic gamma rays is placed in an infinite homogeneous medium (see Fig. 3.1). Then, at different numbers of mean free path lengths (MFP) away from the source, the energy response per $\mathrm{MeV}$ of the photons over a range of energies was computed along with their total energy buildup factor $\left(B_{e}\right)$. The response is equal to the photon energy flux at a radial distance $r$ from the source multiplied by the factor $4 \pi r^{2} e^{\mu r} \cdot \mu$ is the inverse mean free path length of source energy photons in the given medium. When photon mean free pathlengths are stated in this problem, they are mean free paths of the source-energy photons in the given medium. The energy buildup factor is the energy carried by all the photons observed at a distance $r$ from the source divided by the energy carried there by the photons that underwent no collisions (actual energy/uncollided energy).

Goldstein and Wilkins performed their moments method calculations of this problem under the following simplified physics assumptions: ${ }^{15}$

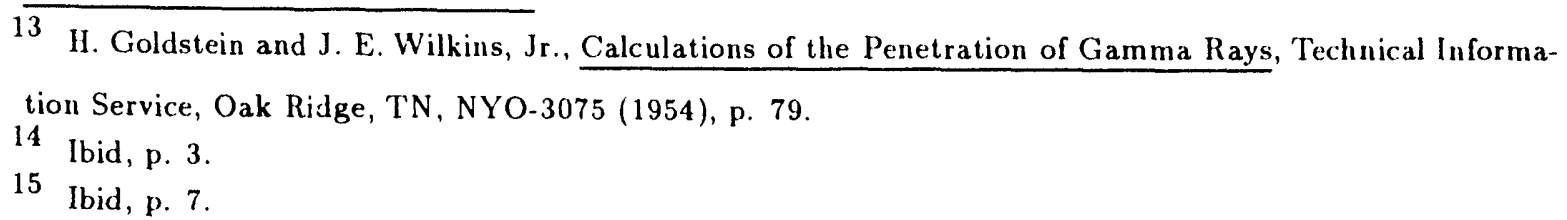




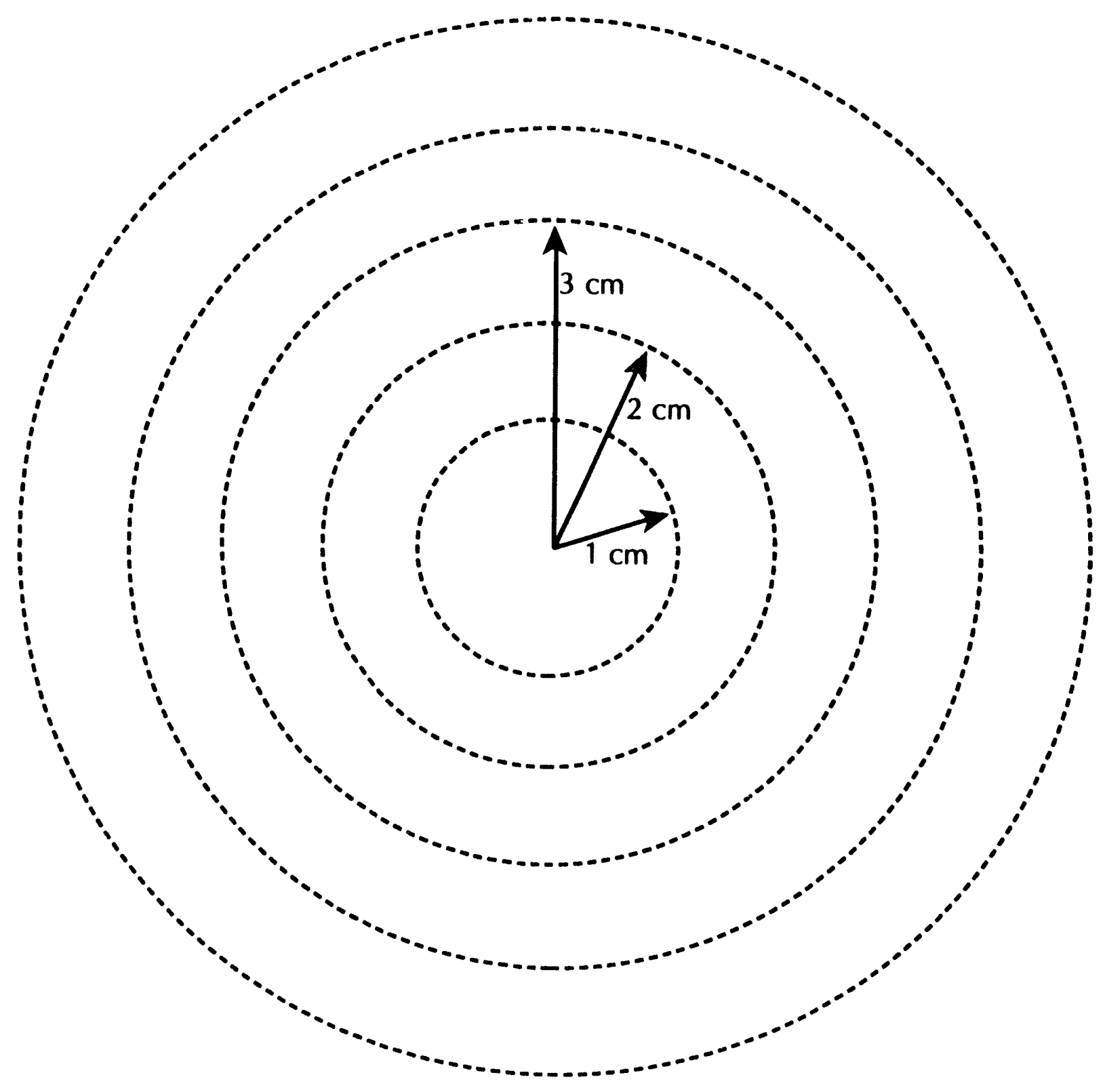

Fig. 3.1. The geometry of the point source in an infinite medium. 
1. Photoelectric and pair production events were treated as pure absorptions. Each process was assumed to occur with its energy-dependent cross section, but no secondary photons were assumed to be produced by the resultant electrons.

2. Coherent (Thomson) scattering did not take place so $\sigma_{c o h}$ consequently was zero.

3. Compton scattering events were assumed to occur with their energy-dependent cross sections, but the resultant electrons again were assumed to produce no secondary photons. Compton scattering was computed without including form factors.

4. No other types of photon interactions occurred. ${ }^{16}$

This problem was chosen as a benchmark in part because it has an analytical solution that allows any potential small MCNP computational errors to be readily detected. It was also chosen to test MCNP's ability to selectively include or ignore different elements of photon/electron physics without modifying the code. MCNP was used to compute the differential energy response and energy buildup factor at $1,2,4$, and $7 \mathrm{MFP}$ in four cases: aluminum and lead, each at $1.0 \mathrm{MeV}$ and 10.0 $\mathrm{MeV}$. The input file for this benchmark is found in Table A.4 of the Appendix.

\section{B. MCNP Problem Model}

1. MCNP Geometry. A point isotropic source of gamma rays (either 1.0 $\mathrm{MeV}$ or $10.0 \mathrm{MeV}$ ) was placed at the origin of a coordinate system. A spherical cell with a 1 MFP radius (for source-energy photons in the given material) was centered at the origin. Thirteen additional cells, each defined to be the region between two concentric spheres, were then centered around the origin and the spherical cell. The first ten shell 'ells were one MFP thick, while the 11th, 12th, and 13th cells were 2, 3, and 4 MrP thick, respectively. The problem boundary was the outermost sphere (of $20 \mathrm{MFP}$ radius), and the region beyond it was made a void. The cells were then specified to be all filled with lead or aluminum, depending on the case being studied.

16 Ibid, p. 7. 
2. Cross Sections/Material Compositions. The mean free paths of 1.0 $\mathrm{MeV}$ and $10.0 \mathrm{MeV}$ photons in $\mathrm{Al}$ and $\mathrm{Pb}$ are determined by the atom densities and total photon cross sections of each as follows:

$$
\begin{aligned}
1 \operatorname{MFP}(\text { in } \mathrm{cm})= & \left\{\left[\sigma_{\text {tot }} \text { (in barns at the source energy }\right)\right] \\
& \left.\left.\times\left[\text { atom density (of } \mathrm{Al} \text { or } \mathrm{Pb} \text { in } 10^{24} \text { atoms } / \mathrm{cm}^{3}\right)\right]\right\}^{-1}
\end{aligned}
$$

where

$$
\sigma_{\text {tot }}=\sigma_{\text {pair prod. }}+\sigma_{\text {photoel. }}+\sigma_{c o m p t}
$$

The total cross section for each case was found in the MCNP MCPLIB cross-section data file and are as follows:

$$
\begin{array}{lccc}
\text { Aluminum: } Z=13 & & \\
& \frac{\mathrm{E}_{\gamma}=1.0 \mathrm{MeV}}{} & & \mathrm{E}_{\gamma}=10.0 \mathrm{MeV} \\
\sigma_{\text {compt. }} & 2.74582 \mathrm{~b} & & 0.66495 \mathrm{~b} \\
\sigma_{\text {pair } / \text { prod. }} & 0.00100 \mathrm{~b} & & 0.00004 \mathrm{~b} \\
\sigma_{\text {photoel. }} & \underline{0.0 \quad \mathrm{~b}} & & \frac{0.37344 \mathrm{~b}}{1.03843 \mathrm{~b}} \\
\sigma_{\text {tot }} & 2.74682 \mathrm{~b} & & 1.0304
\end{array}
$$

Lead: $Z=82$

\begin{tabular}{lccr} 
& $\mathrm{E}_{\gamma}=1.0 \mathrm{MeV}$ & & $\mathrm{E}_{\gamma}=10.0 \mathrm{MeV}$ \\
\cline { 2 - 2 }$\sigma_{\text {compt. }}$ & $17.18180 \mathrm{~b}$ & & $4.19291 \mathrm{~b}$ \\
$\sigma_{\text {pair/prod. }}$ & 6.02800 & & $0.16809 \mathrm{~b}$ \\
$\sigma_{\text {photoel. }}$ & $\underline{0.0}$ & & $\frac{12.40100 \mathrm{~b}}{16.76200 \mathrm{~b}}$ \\
$\sigma_{\text {tot }}$ & $23.20980 \mathrm{~b}$ & &
\end{tabular}

The resultant mean free paths are:
Al : $\quad 1.0 \mathrm{MeV}:$
$1 \mathrm{MFP}=\underline{6.044 \mathrm{~cm}}$
$10.0 \mathrm{MeV}$ :
$1 \mathrm{MFP}=\underline{15.986 \mathrm{~cm}}$
$\mathrm{Pb}: \quad 1.0 \mathrm{MeV}$ :
$1 \mathrm{MFP}=\underline{1.306 \mathrm{~cm}}$,
$10.0 \mathrm{MeV}$ :
$1 \mathrm{MFP}=1.809 \mathrm{~cm}$ 
Note that this problem is independent of photon data library, but if a different cross section is used, then the geometry must be changed to accommodate the different mean free path sphere spacing.

3. Code Tallies. Next, surface tallies for photon energy flux (*F2:P tallies, whose units are $\mathrm{MeV} / \mathrm{cm}^{2}$ ) were placed on the 1, 2, 4, and 7 MFP spheres. The tally results were distributed among energy bins for all four tallies. The response/MeV (or differential energy response) was obtained by dividing the tally result in each bin by the width of the bin in $\mathrm{MeV}$ and then multiplying each bin result by $4 \pi \mathrm{r}^{2} \mathrm{e}^{\mu r}$ with $\mathrm{r}$ in $\mathrm{cm}$ ). This calculation was done using an EM card whose multiplicative factor for each bin of each tally was

$$
\frac{4 \pi r^{2} e^{\mu r}}{\text { bin width }(\mathrm{MeV})}
$$

Two more energy flux surface tallies were each placed on $1: 1,2,4$, and 7 MFP spheres to compute the energy buildup factor there. The first tally was used only to calculate the energy flux at each sphere. The second tally also calculated the energy flux at each distance but binned its results according to how many collisions the tallied photon had undergone before reaching each distance. This calculation was done by using the MCNP FT/FU card inc options:

\section{FT 122 INC}

\section{FU $12201234561000000 \mathrm{~T}$}

The talliea photon flux in the zero bin above is caused by those photons which reach each surface without having collided at all. The erergy flux from the first tally divided by the uncollided energy flux in the first bin of the second tally is the ratio of the actual energy to the uncollided energy: the energy buildup factor.

4. Variance Reduction. After the tallies were arranged, importances were assigned to each cell. This assignment was necessary to optimize the computational efficiency of all four problems. Sufficient photon attenuation occurred by 7 MFP away from the source to heavily impair tally efficiency there. The importances for each cell were initially set to increase by a facior of two for each MFP between the source and the inner surface of the cell. Then they were adjusted by trial and error to produce roughly equal particle populations in each cell. 
5. Code Physics. Finally, the photon/electron physics options were specified in the problem by using the PHYS:P and PHYS:E cards:

PHYS:P $\quad .001 \quad 0 \quad 1$

PHYS:E 8J 0

The PHYS:P card turns off coherent scattering thus restricting the photon physics to

1. pair production

2. photoelectric effect

3. Compton scattering (no form factors)

The PHYS:E card allows the production of electrons from the interactions above but then effectively removes them from the problem as if photoelectric and pair production were pure absorption.

\section{Resilts and Discussion}

The MCNP computed results of the differential energy response for $\mathrm{Al}$ and $\mathrm{Pb}$ for $1.0 \mathrm{MeV}$ at 1 and $7 \mathrm{MFP}$ are found in Figs. 3.2 to 3.5 and are plotted with the corresponding Goldstein and Wilkins ${ }^{17}$ data as a function of energy in each case. In all the cases (including those not graphed here), MCNP yields differential responses within one standard deviation of the analytical results in $60-70 \%$ of the data - the statistically expected agreement for tallies whose estimates are independent.

However, as in benchmarks one and two, there is some correlation between the estimates of this problem's tallies. Therefore, these statistics must be interpreted in the same way as those in the first two benchmarks (see the statistical interpretation sections of benchmarks one and two). Results show that MCNP is in good agreement with the analytical results for the differential responses. The MCNP energy buildup results are found in Tables 3.1 and 3.2 with the Goldstein and Wilkins results $^{18}$ and the COG data. ${ }^{19}$ In 15 out of 16 instances, MCNP calculated an energy

17 Ibid, pp. 90-93, pp. 106-109.

18 Ibid, p. 136 and p. 140.

19 Wilcox and Lent (Ref. 8), p. 45. 


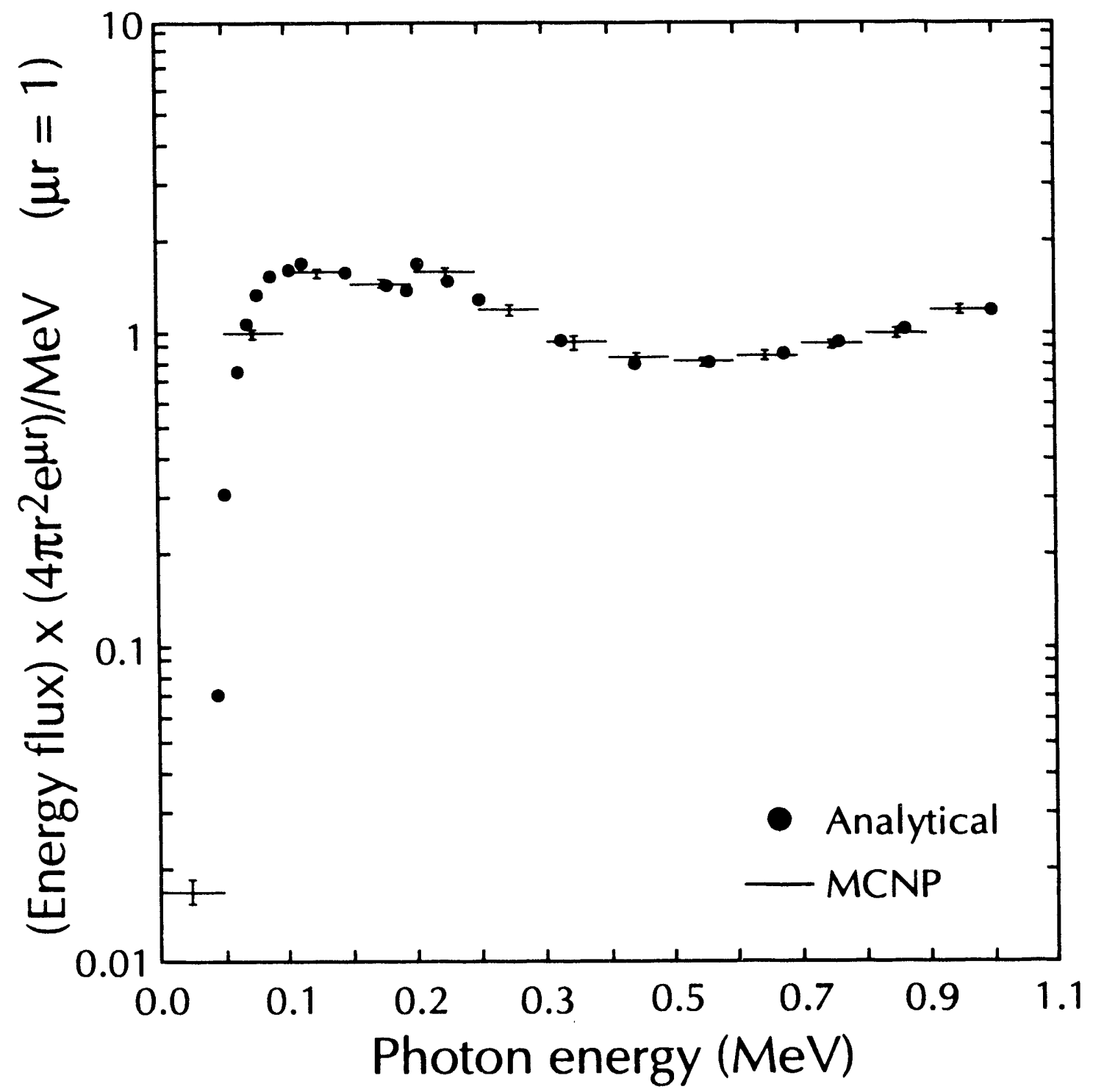

Fig. 3.2. $\mathrm{Al}$ at $1.0 \mathrm{MeV}$ and $1 \mathrm{MFP}$. 


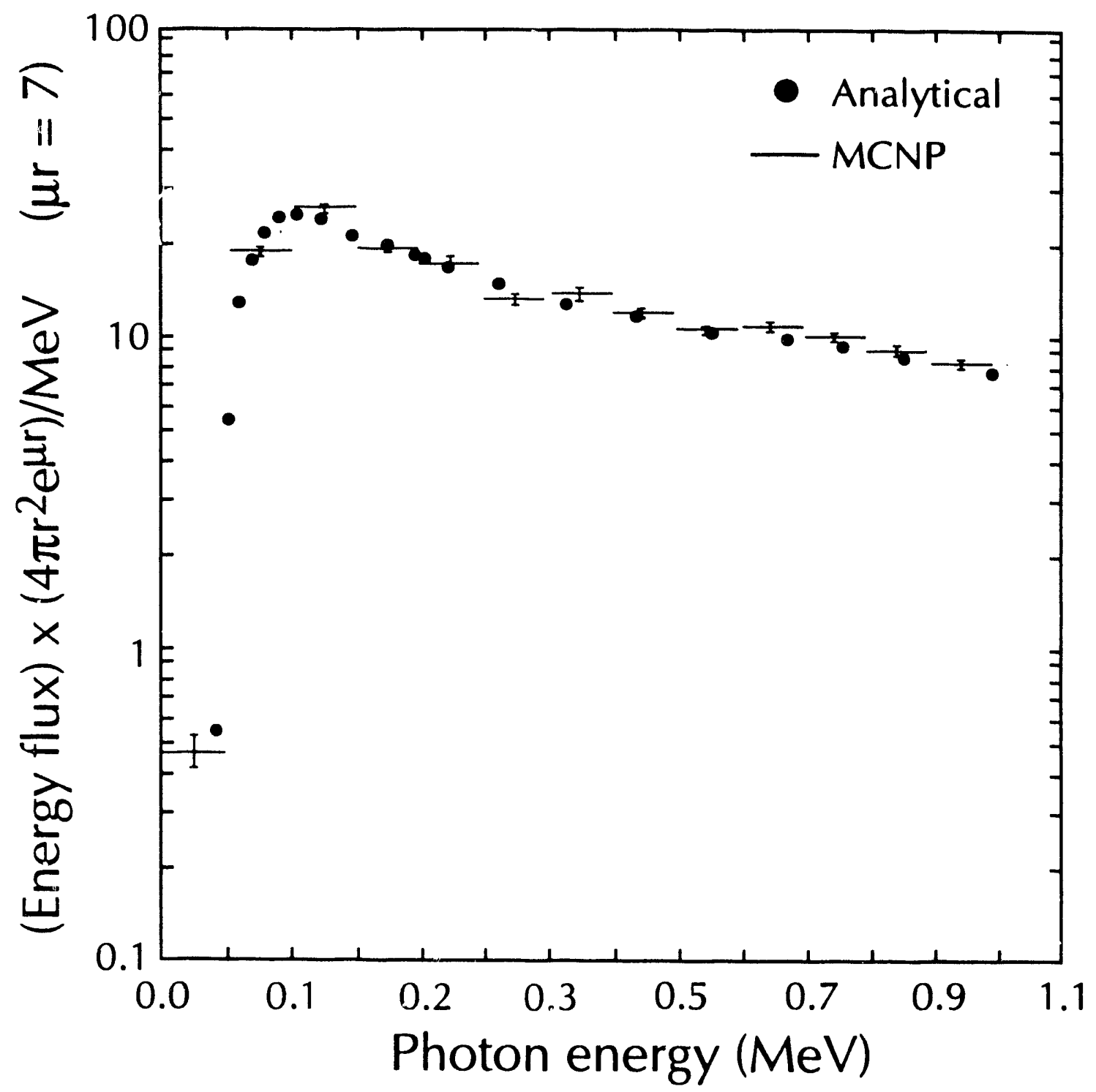

Fig. $3.3 \mathrm{Al}$ at $1.0 \mathrm{MeV}$ and $7 \mathrm{MFP}$. 


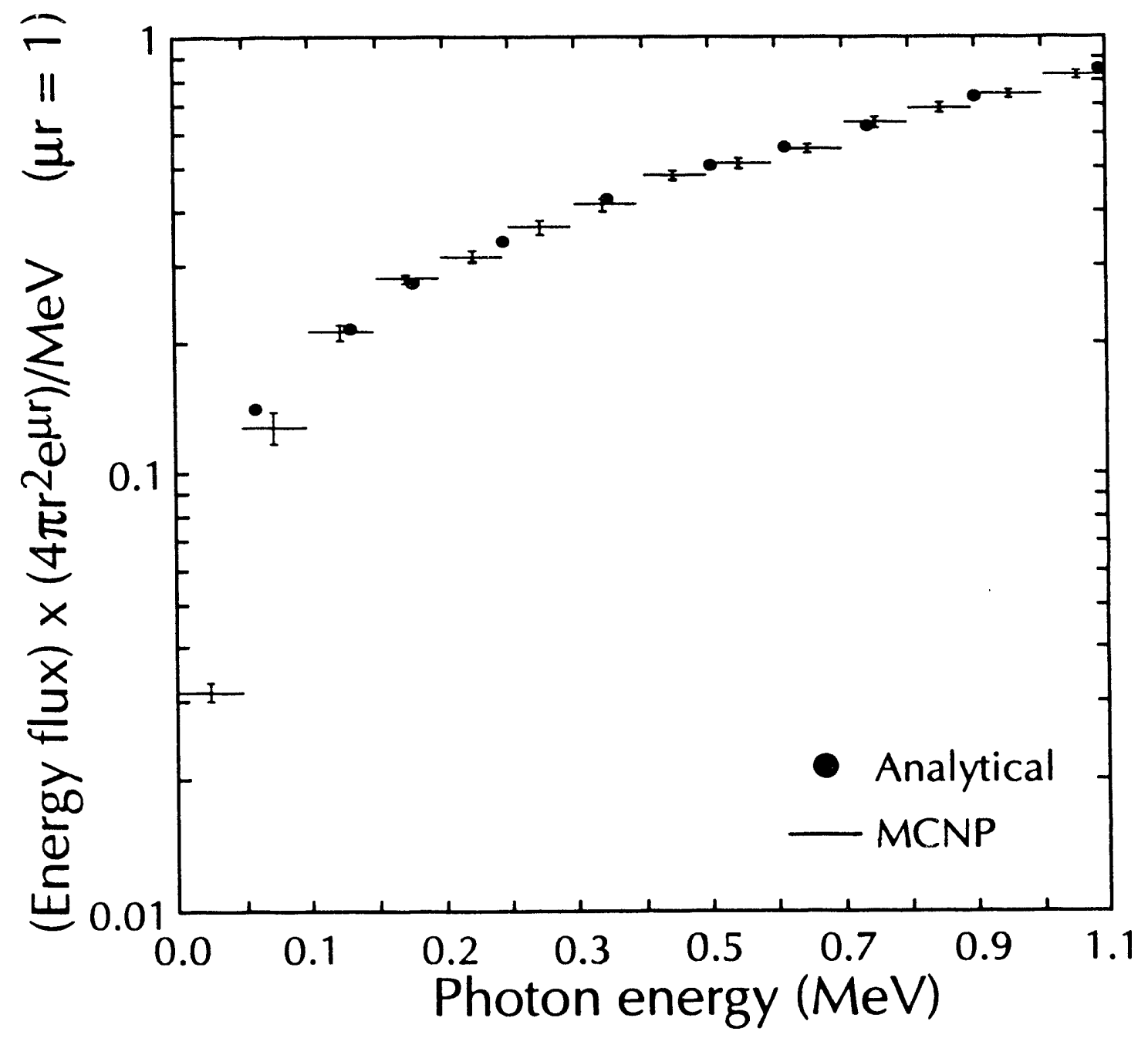

Fig. 3.4. $\mathrm{Pb}$ at $1.0 \mathrm{MeV}$ and $1 \mathrm{MFP}$. 


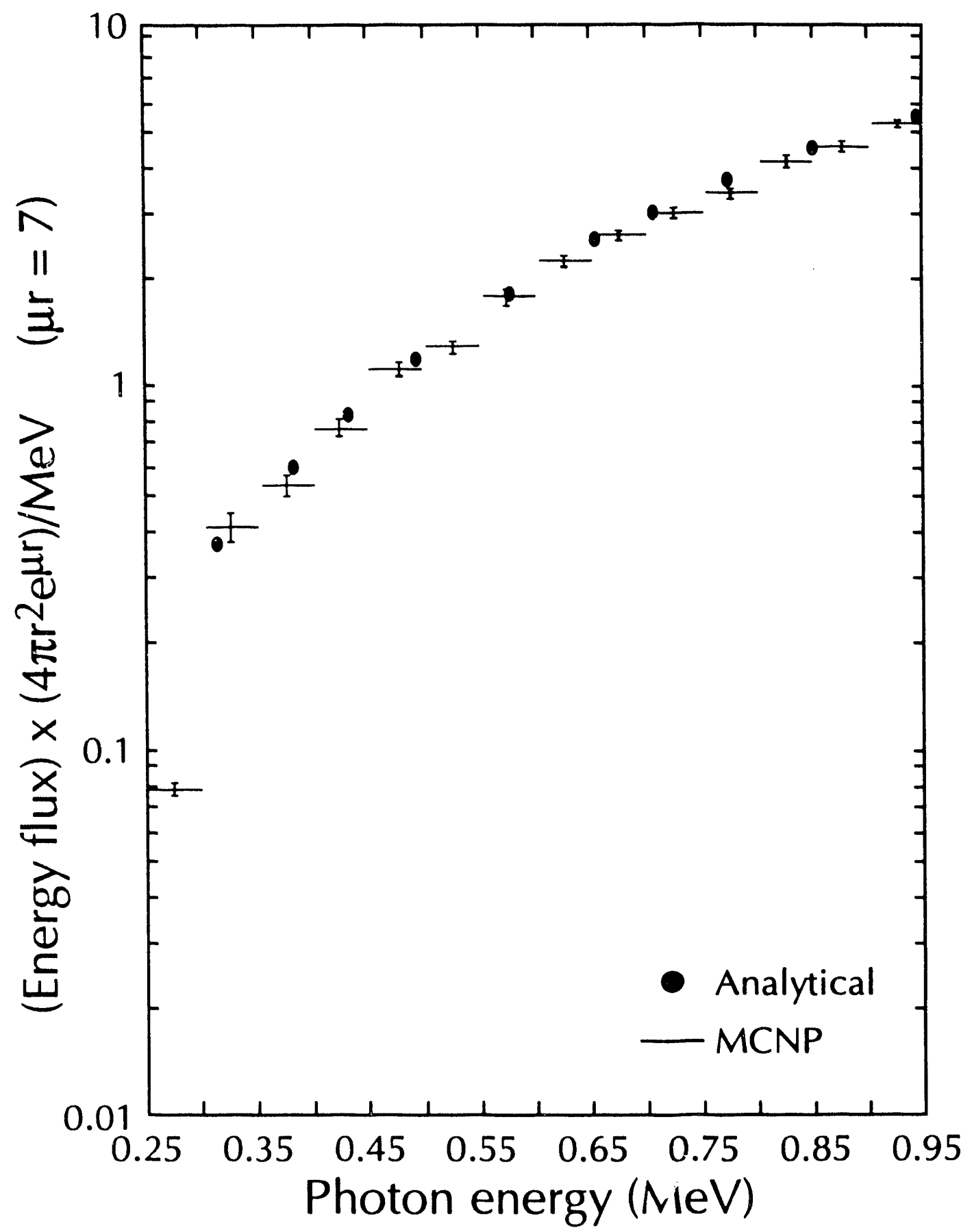

Fig. 3.5. $\mathrm{Pb}$ at $1.0 \mathrm{MeV}$ and $7 \mathrm{MFP}$. 
TABLE 3.1

THE ENERGY BUILDUP $\left(B_{\epsilon}\right)$ OF GAMMA RAYS FROM A POINT SOURCE IN AN INFINITE MEDIUM OF Al at 1.0 and $10.0 \mathrm{MeV}$

\begin{tabular}{rrrrr}
\hline MeV & $\begin{array}{c}\text { Mean Free } \\
\text { Path } \\
\text { (MFP) }\end{array}$ & Analytic & MCNP & COG \\
\hline & & & & \\
1 & 1 & 2.01 & $2.018 \pm 0.020$ & $2.021 \pm 0.036$ \\
& 2 & 3.29 & $3.307 \pm 0.059$ & $3.303 \pm 0.052$ \\
& 4 & 6.52 & $6.648 \pm 0.254$ & $6.466 \pm 0.140$ \\
& 7 & 12.95 & $12.622 \pm 0.936$ & $12.306 \pm 0.610$ \\
10 & 1 & 1.22 & $1.227 \pm 0.013$ & $1.22 .4 \pm 0.014$ \\
& 2 & 1.45 & $1.460 \pm 0.029$ & $1.468 \pm 0.019$ \\
& 4 & 1.91 & $1.944 \pm 0.081$ & $1.977 \pm 0.046$ \\
& 7 & 2.64 & $2.793 \pm 0.201$ & $2.721 \pm 0.202$ \\
\hline
\end{tabular}

TABLE 3.2

THE ENERGY BUILDUP $\left(B_{e}\right)$ OF GAMMA RAYS FROM A POINT SOURCE IN AN INFINITE MEDIUM OF $P b$ at 1.0 and $10.0 \mathrm{MeV}$

\begin{tabular}{|c|c|c|c|c|}
\hline $\mathrm{MeV}$ & $\begin{array}{c}\text { Mean Free } \\
\text { Path } \\
(\mathrm{MFP}) \\
\end{array}$ & Analytic & MCNP & $\mathrm{COC}$ \\
\hline \multirow[t]{4}{*}{1} & 1 & 1.35 & $1.361 \pm 0.006$ & $1.334 \pm 0.017$ \\
\hline & 2 & 1.66 & $1.650 \pm 0.013$ & $1.605 \pm 0.025$ \\
\hline & 4 & 2.21 & $2.186 \pm 0.028$ & $2.134 \pm 0.060$ \\
\hline & 7 & 2.95 & $2.901 \pm 0.058$ & $2.893 \pm 0.205$ \\
\hline \multirow[t]{4}{*}{10} & 1 & 1.09 & $1.089 \pm 0.0062$ & $1.086 \pm 0.013$ \\
\hline & 2 & 1.19 & $1.192 \pm 0.0096$ & $1.192 \pm 0.016$ \\
\hline & 4 & 1.46 & $1.478 \pm 0.0179$ & $1.153 \pm 0.030$ \\
\hline & 7 & 2.16 & $2.255 \pm 0.0438$ & $2.011 \pm 0.113$ \\
\hline
\end{tabular}


buildup within the MCNP relative error of the analytical data. These data show that MCNP successfully models these gamma ray penetration problems within the statistical uncertainty inherent in the Monte Carlo method.

\section{BENCHMARK PROBLEM FOUR - GAMMA-RAY SKYSHINE EXPERIMENT}

\section{A. Problem History \& Description}

Interest in the computation of gamma-ray exposure rates in air at large distances from concentrated gamma sources has arisen because air-scattered photon radiation (commonly referred to as "skyshine") arouses concern in the design of nuclear installations. ${ }^{20}$ Until 1980 , most skyshine studies were concerned with fallout fields or involved complicated geometries that were difficult to model. ${ }^{21}$ As a result, it was difficult to assess the accuracy of transport code models of skyshine fields from concentrated gamma sources.

Concern over the adequacy of such code models prompted R. R. Nason, J. K. Shultis, R. E. Faw, and C. E. Clifford to conduct a skyshine benchmark experiment at a shielding research facility in the Kansas plains in $1980 .^{22}$ In this experiment, a collimated gamma source was placed in an open field at ground level (see Fig. 4.1). Dose rates and differential flux densities of skyscattered gamma rays were measured by detectors on the ground at $100 \mathrm{~m}$ intervals from the source out to $700 \mathrm{~m}$ (see Fig. 4.2). These measured dose rates and flux spectra were then compared to a DOT discrete ordinates code model of the experiment.

MCNP was used to model only the dose rates measured in the gamma skyshine benchmark. This experiment was chosen for study because it involves the deep penctration of gamma rays. It was also chosen because of its relevance to the nuclear engineering shielding community. MCNP's results for the dose rates were compared to the measured data.

\section{B. MCNP Problem Model}

1. Experimental Arrangement. In the skyshine experiment, a ${ }^{60} \mathrm{Co}$ gamma source (1.33 and $1.17 \mathrm{MeV}$ ), which was collimated to cmit photons isotropically into

20 R. R. Nason, J. K. Shultis, R. E. Faw, and C. E. Clifford, "A Benchmark Gamma-Ray Skyshine Experiment," Nuclear Science and Engincering, 79, (1981), p. 404.

21 llid, p. 404.

2 Ibid, p. 404. 


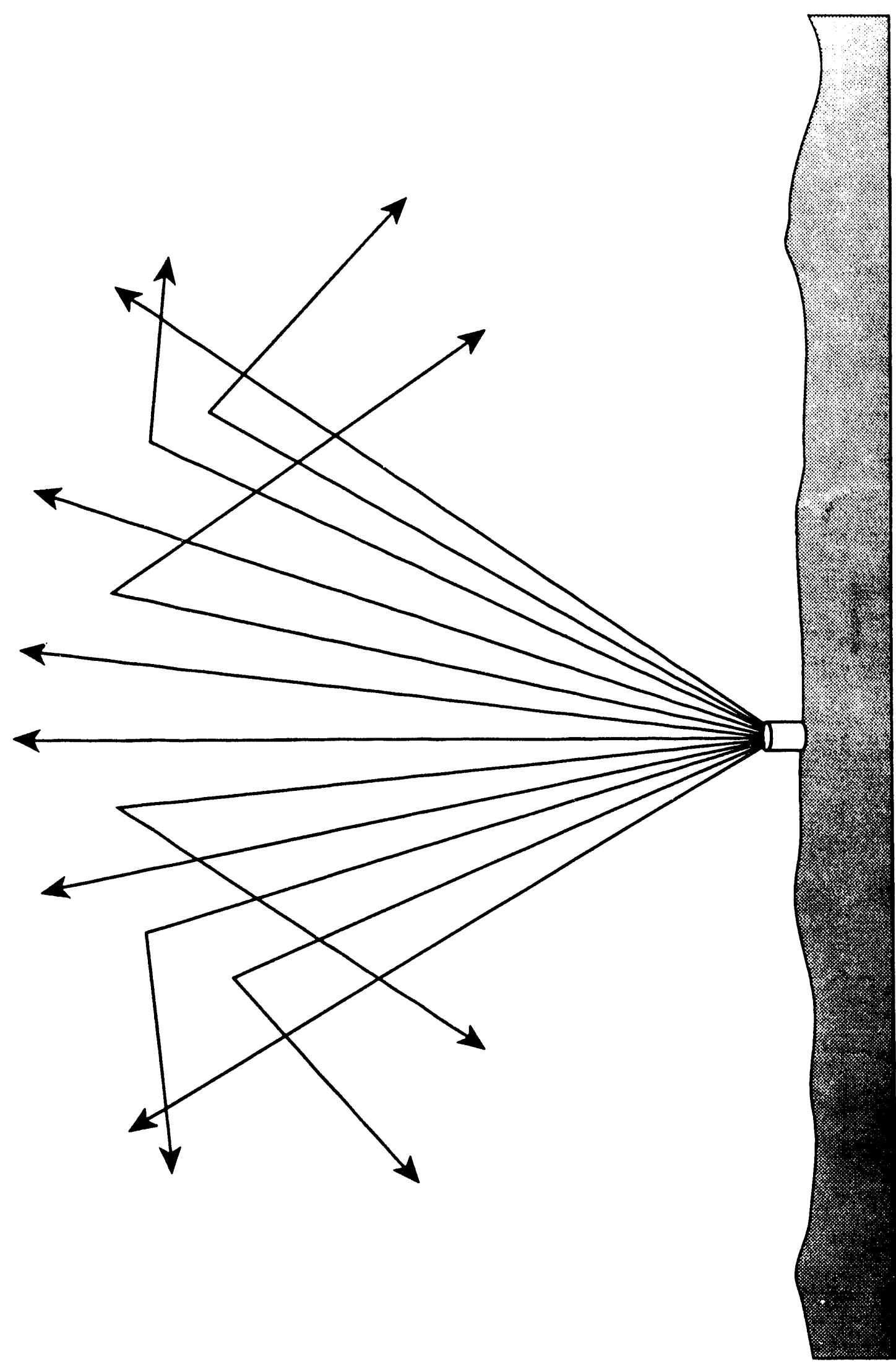

34 


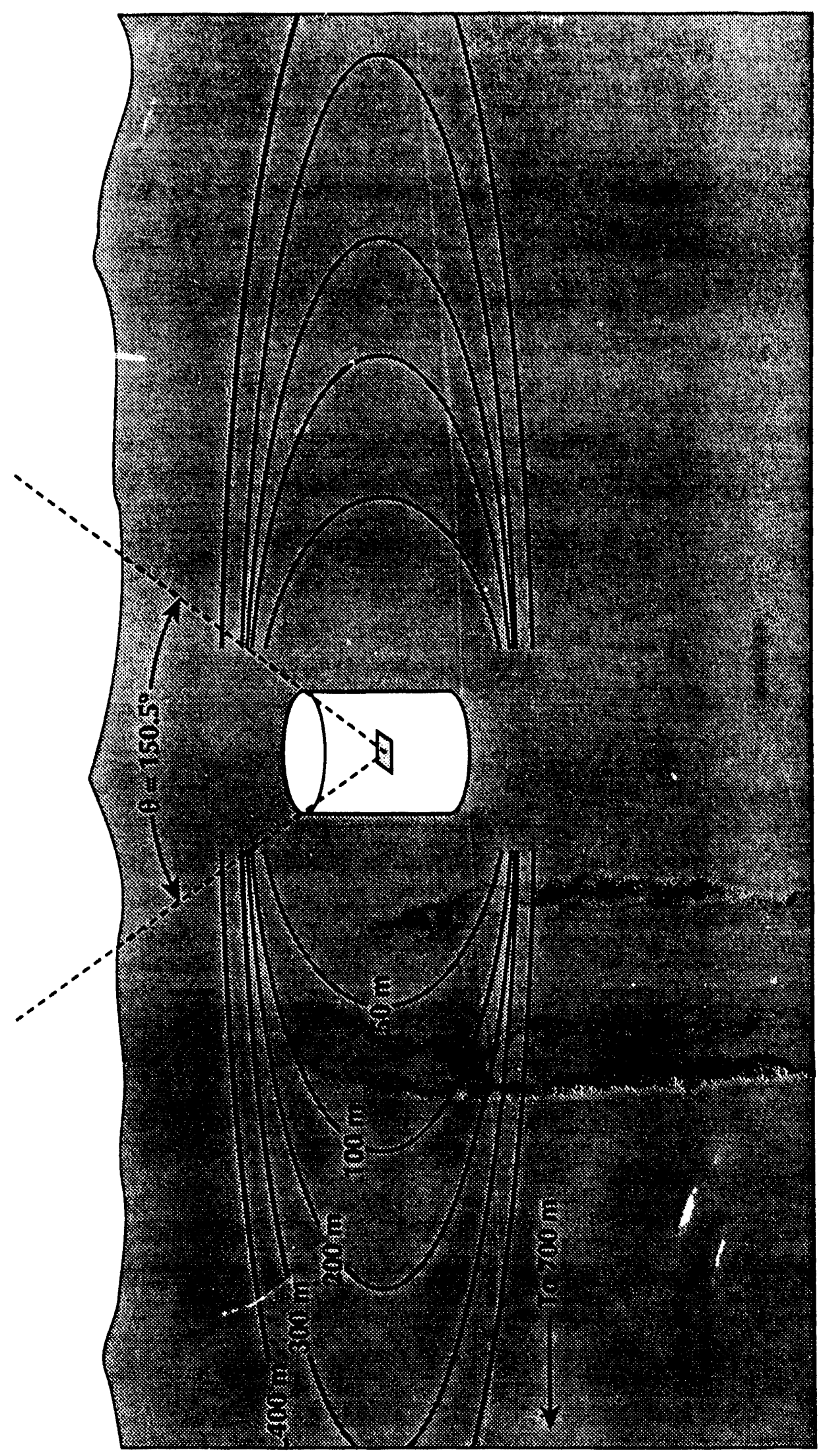

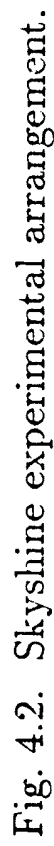


a $150.5^{\circ}$ vertical cone, was placed in an open area. Gamma detectors were placed approximately one meter above the ground at $50 \mathrm{~m}, 100 \mathrm{~m}$, and at $100 \mathrm{~m}$-intervals thereafter out to $700 \mathrm{~m}$ from the collimated source. Two types of detectors were used: NaI for spectral and rate measurements, and high pressure ion chambers (HPIC) for rate measurements only. The open field where this experiment was conducted had rises and depressions: the maximum detector elevation relative to the source was $2.51 \mathrm{~m}$, and the average elevation was $1.39 \mathrm{~m}^{23}$

2. Problem Boundary/Material Compositions. The first step to modeling the skyshine experiment was setting up the problem boundary. A 1-km radius sphere was placed at the origin of a cartesian coordinate system and sliced into two hemispheres by a plane coincident with the XY-plane. Three additional planes parallel to the XY plane were then placed 3,6, and $9 \mathrm{~cm}$ below it. The $\mathrm{XY}$-plane was designated to be the ground/air interface.

The ground surface was represented by a plane because the terrain of the experiment was not precisely specified. The ground (enclosed by the XY-plane, the plane at $-9 \mathrm{~cm}$, and the $1-\mathrm{km}$ sphere) was filled with soil. The soil composition and density in the experimental area were never specified, so a standard soil elemental composition with a density of $2.6 \mathrm{gm} / \mathrm{cm}^{3}$ was used in the model. The upper hemisphere bounded by the XY-plane and the $1-\mathrm{km}$ sphere was filled with air. The air density in the experiment was not specified, so a standard composition at 0.001124 $\mathrm{g} / \mathrm{cm}^{3}$ was used in the model.

A $1-\mathrm{km}$ boundary was chosen for this problem because $1000 \mathrm{~m}$ is approximately ten $1.33 \mathrm{MeV}$ photon mean free pathlengths in air. MCNP weight window calculations indicated that photons backscattered to detectors from beyond this distance would make a negligible contribution to the measured dose rates. After the problem boundary and material compositions were chosen, the MCNP geometry of the collimated gamma source was created.

3. Experimental Source. The experimental source consisted of a point ${ }^{60} \mathrm{Co}$ gamma source in a cask placed on the axis of an annular (actually dodecahedral, or 12-sided) concrete silo. ${ }^{24}$ This silo, $2.29 \mathrm{~m}$ high, had a maximum inner diameter of $2.50 \mathrm{~m}$ and a maximum outer diameter of $4.35 \mathrm{~m}$. The source was placed 1.98 meters above the base of the silo (which was on the ground). Lead and concrete

\footnotetext{
23 Ibid, p. 407.

24

Ibid, p. 405.
} 
blocks were then placed along the top of the silo so that uncollided photons from the source would exit the top of the silo in a $150.5^{\circ}$ vertical cone.

In spite of the measures taken to ensure that the gamma photons would leave the silo isotropically in a cone, in-silo scattered photons partially distorted the cone radiation pattern. Some of the scattered photons leaked through the silo walls, and others scattered out the silo top but outside the cone. Because this in-silo scattered gamma component could not be cliaracterized, it was not possible to model the experimental source exactly. Nevertheless, it was possible to model the originally-intended isotropic cone source with MCNP.

4. Modeled Source. The source silo was modeled by a cell which was defined to be the region between two concentric cylinders capped by two planes perpendicular to the cylinders' axes (see Fig. 4.3). The resultant annular silo cell, $2.29 \mathrm{~m}$ high, had an inner radius of $117.75 \mathrm{~cm}$ and an outer radius of $217.5 \mathrm{~cm}$. This silo cell's lower base was centered at the origin on the XY-plane. The silo cell volume and the ground layer disk directly beneath the silo were made voids.

An isotropic point source which emitted 1.332 and $1.173 \mathrm{MeV}$ photons with equal probability was then placed $1.98 \mathrm{~m}$ above the ground on the silo's axis. The volume enclosed by the silo (but not inside the silo cell volume itself) was filled with air. This source geometry guaranteed that the source photons would leave the silo in a $150.5^{\circ}$ isotropic cone. There was no in-silo scatter component because the silo walls and base were regions of zero importance: any photons that struck these regions were terminated there.

5. Cell Subdivision. After the problem boundary, air, and soil regions were defined along with the silo cell and collimated source, the problem geometry was further subdivided into cells. The regions that were directly irradiated by the source (i.e., within the source cone) were partitioned into spherical-shell layer cells bounded by the source cone (see Fig. 4.4). The regions above the ground that received only scattered radiation were partitioned into segmented conical shell cells which were parallel to and radiated out from the source cone. The regions beneath the ground that received scattered gamma rays were slice $l$ into three flat disk cells (see Fig. 4.5). Complete details of the cell geometry are found in the MCNP input file for this experiment in Table A.5 in the Appendix.

6. Code Tallies. Eight concentric ring detectors centered at the origin were next placed $1.0 \mathrm{~m}$ above and parallel to the air-ground interface. The ring detectors had 


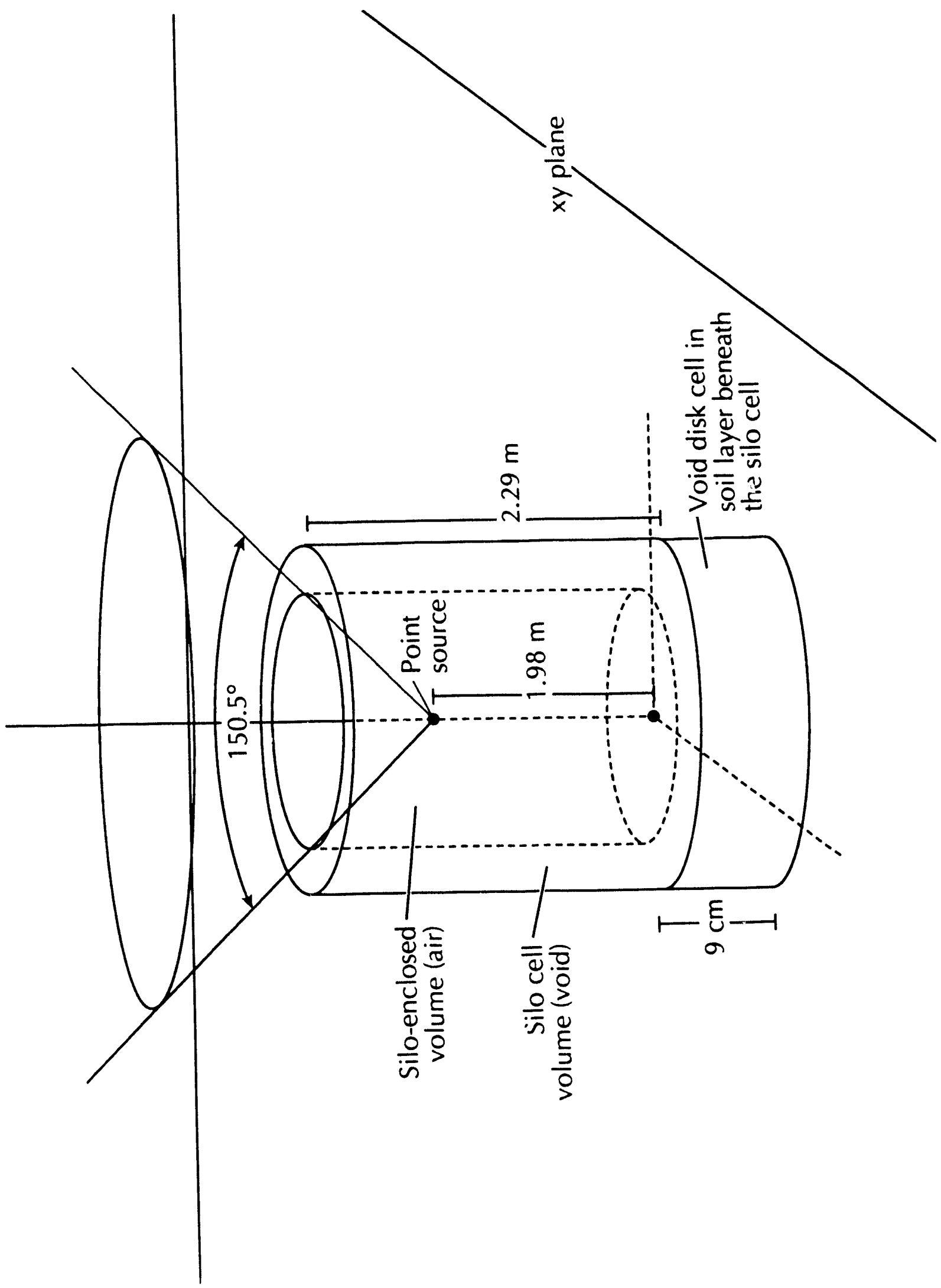

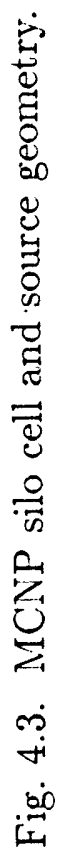




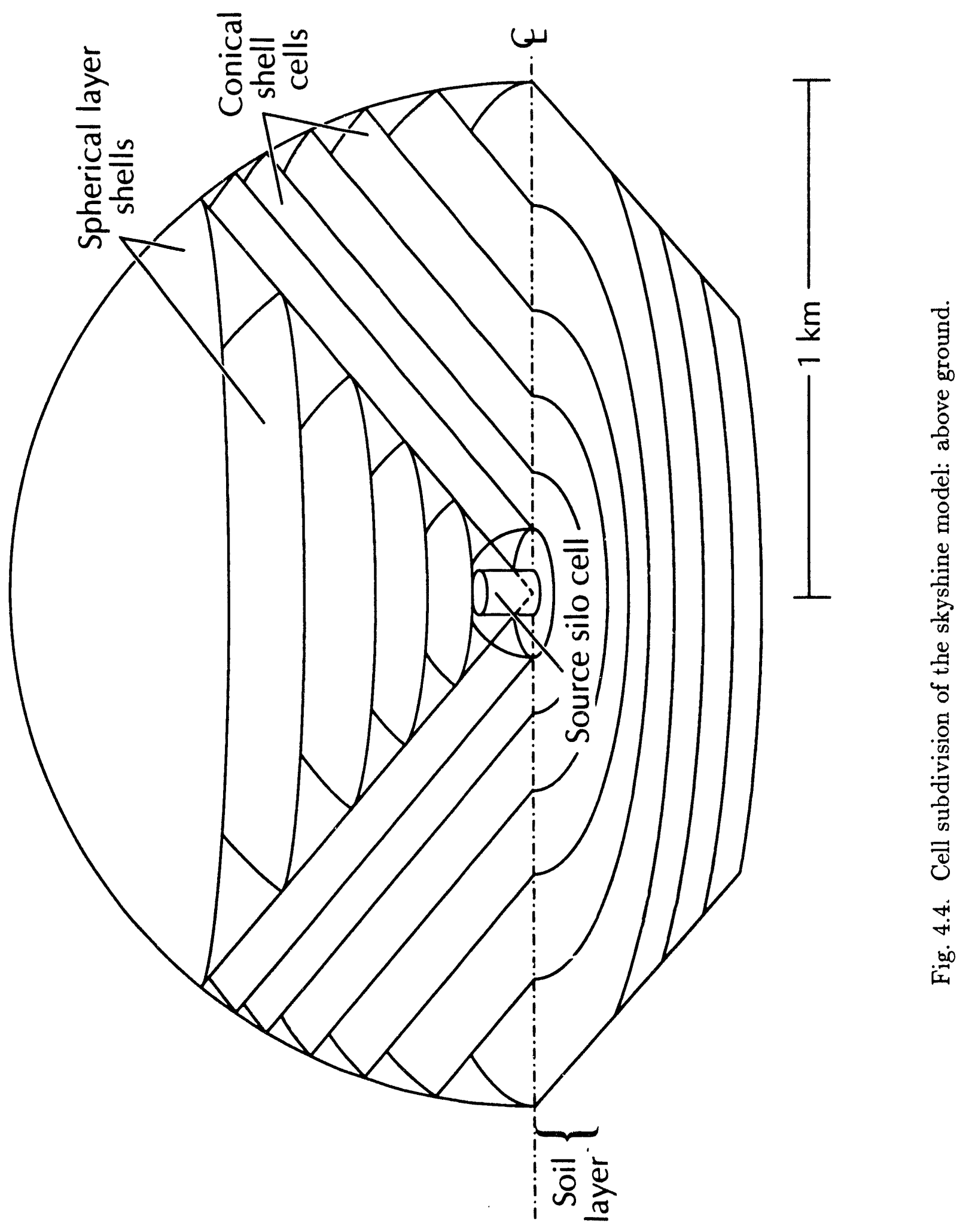




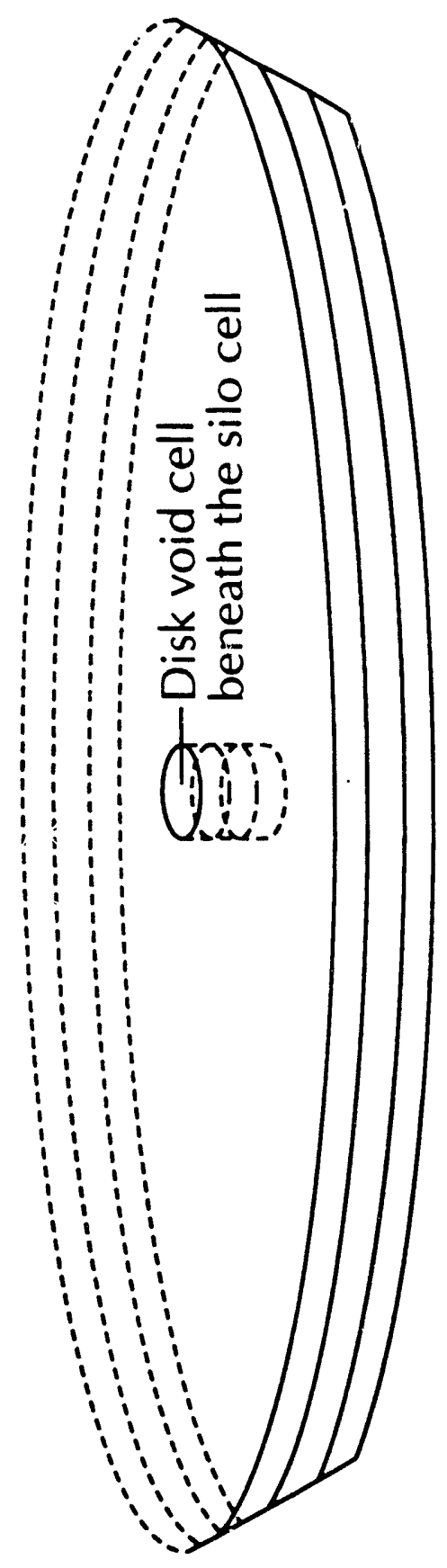

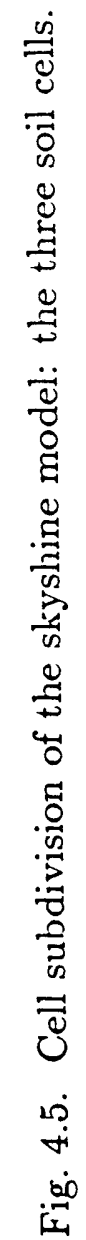


radii corresponding to the distances that the experimental detectors were located from the source: $50 \mathrm{~m}, 100 \mathrm{~m}$, and $200 \mathrm{~m}$ to $700 \mathrm{~m}$ at $100 \mathrm{~m}$ intervals. The flux estimate of each detector was modified by an FM card to obtain the energy deposited per unit volume in air per photon history $\left(\mathrm{MeV} / \mathrm{cm}^{3} \cdot\right.$ history).

After the code tallies were established, importances were assigned to each cell.

7. Variance Reduction/Code Physics. Importances were assigned to the cells to equalize their particle populations and improve sampling. Optimizing the importances to accomplish this was done by trial and error. A CUT:P option was then used to terminate photons with energies below a $39.9 \mathrm{KeV}$ cutoff. This prevented MCNP from wasting time following photons whose energies were below the detector response function in the experiment. After this option was used, the input file was run to obtain the MCNP results for this experiment.

\section{Results and Discussion}

1. MCNP Tally Conversion. The MCNP ring detector estimates (modified to yicld $\mathrm{MeV} / \mathrm{cm}^{3} /$ particle history) had to be converted to the $\mu / \mathrm{rad} /$ hour/Curie units of the experimental data before any model comparison could be made. This conversion was accomplished in two steps. First, the MCNP estimates were changed to units of rad/history as follows:

$$
\begin{gathered}
\operatorname{MCNP}(\mathrm{rad} / \text { history })= \\
\operatorname{MCNP}\left(\frac{\mathrm{MeV}}{\mathrm{cm}^{3} \cdot \text { history }}\right) \times\left\{\frac{\left(1.602 \times 10^{-6} \frac{\mathrm{erg}}{M \mu \mathrm{eV}}\right) \cdot\left(1 \mathrm{rad} / 100 \frac{\mathrm{erg} g}{g}\right)}{\rho_{\text {air }}\left(\frac{\mathrm{g}}{\mathrm{cm}^{3}}\right)}\right\}
\end{gathered}
$$

The tally estimates were then converted from rad/history to $\mu \mathrm{rad} /$ hour/Curie by

$$
\begin{gathered}
\operatorname{MCNP}(\mu \mathrm{rad} / \mathrm{hr} / \mathrm{Ci})= \\
\operatorname{MCNP}(\mathrm{rad} / \text { history }) \times\left\{\left(3.7 \times 10^{10} \frac{\mathrm{hist} .}{\mathrm{sec}} / \mathrm{Ci}^{\mathrm{i} i}\right) \times\left(3600 \frac{\mathrm{sec}}{\mathrm{hr}}\right) \times\left(10^{6} \frac{\mathrm{\mu rad}}{\mathrm{rad}}\right)\right\}
\end{gathered}
$$

The two steps can be combined into a single conversion:

$\operatorname{MCNP}(\mu \mathrm{rad} / \mathrm{hr} / \mathrm{Ci})=\operatorname{MCNP}\left(\frac{\mathrm{MeV}}{\mathrm{cm}^{3} \cdot \text { history }}\right) \times\left(1.899 \times 10^{15} \frac{\mu \mathrm{rad} / \mathrm{hr} / \mathrm{Ci}}{\mathrm{MeV} / \mathrm{cm}^{3} \cdot \text { hist }}\right)$ 
The units used in the benchmark paper were necessarily chosen to be independent of the source strength because three different source strengths had to be used to obtain accurate measurements in the experiment: $10.3,229$, and $3800 \mathrm{Ci}^{25} \mathrm{~A}$ source that would provide a reasonable signal for detectors close to the source would not have registered well in the outer detectors. A source that would deliver a measurable signal to the outer detectors would swamp the inner detectors. Consequently, several runs with the three different source strengths were performed to obtain accurate data from all the detectors. The measurements were all normalized per source strength so the data of different source runs could all be compared together.

2. MCNP Results. After the MCNP results were computed and converted to the correct experimental units, they were plotted as a function of $\mathrm{r} \rho_{\text {air }}$, or column density (in grams $/ \mathrm{cm}^{2}$ ) along with the experimental measurements. The data were plotted according to column density to divide out day-to-day variations in atmospheric density (high- and low-pressure systems periodically moved in and out of the experimental area). In Fig. 4.6, the solid line connects the eight MCNP ring detector estimates of the dose rates. The asterisks denote the experimental values.

The experimental data was taken from Table 1 of Nason et al. ${ }^{26}$ Upon inspecting the table, it might be thought that the data of each line of column 5 can be directly calculated from the data in the same line of columns 1,3 , and 4 :

$$
[\text { column } 5 \text { data }]=[\text { column } 1 \text { data }]^{2} \cdot[\text { column } 4 \text { data }] /[\text { column } 3 \text { data }]
$$

However, if this calculation is done, the result is not quite what is found in column 5 . This apparent discrepancy develops because the exposure rate in column 4 for each distance from the source was actually first multiplied by a detector correction factor corresponding to that distance. Then this corrected rate (which never explicitly appears in the table) was combined with columns 1 and 3 as shown above to yield what is actually found in column 5 . These correction factors are found in Table 4 of Nason et al. ${ }^{27}$ The graph in Fig. 4.6 demonstrates that MCNP provides a very good representation of the skyshine measurements given the experimental uncertainty in them.

\footnotetext{
25 Ibid, p. 405.

26 Ibid, p. 411.

27 Ibid, p. 414.
} 


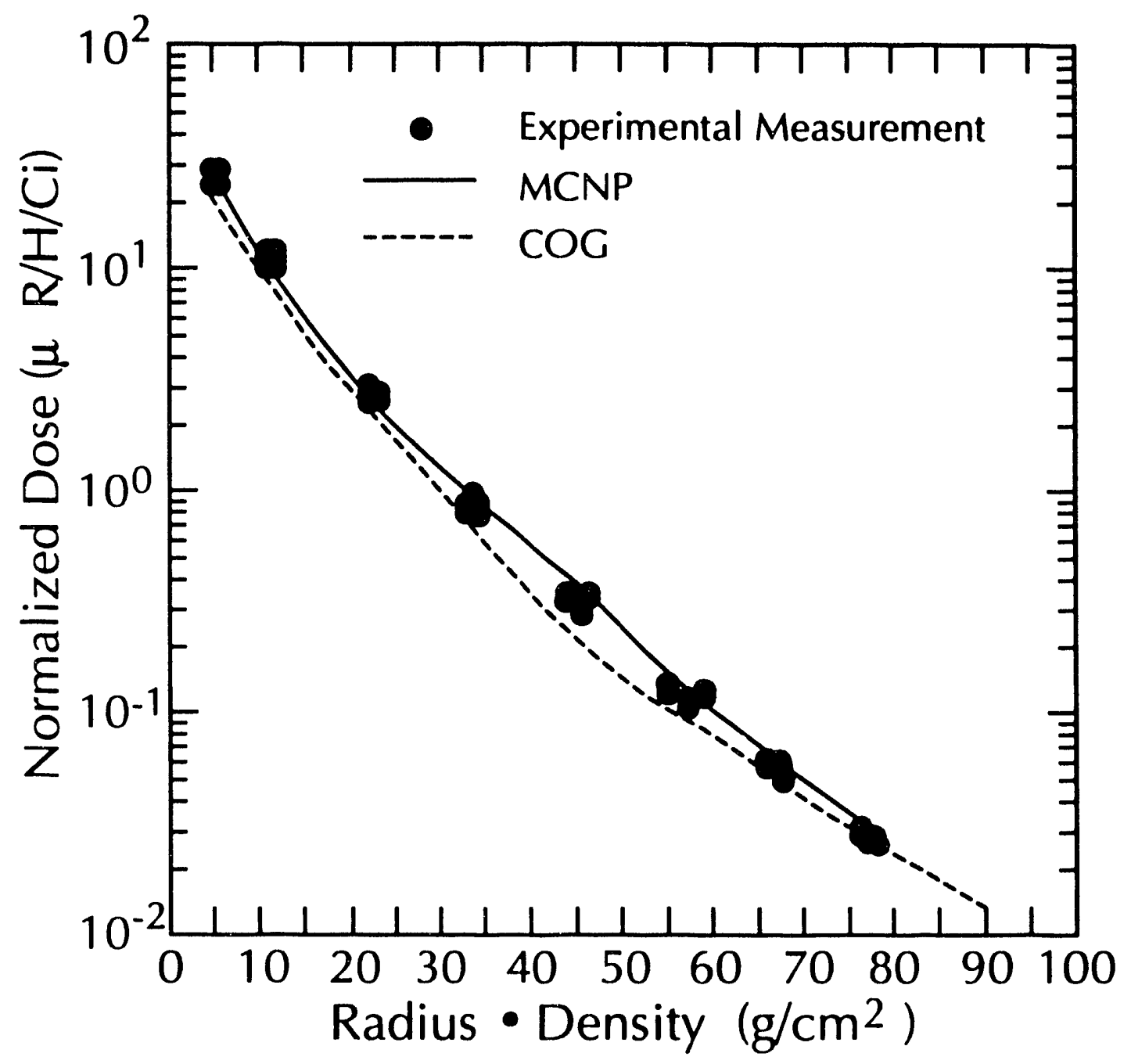

Fig. 4.6. Normalized dose rates of the gamma skyshine experiment. 
3. Experimental Uncertainty. One major source of the uncertainty of the measured data came from the distortion present in the radiation pattern of the experimental source. Nason et al. estimated that the in-silo scatter component of the source probably accounted for most of the 10\%-20\% difference between their measured data and expected data as predicted by the DOT discrete ordinates code. ${ }^{28}$ Next, the terrain where the experiment was conducted had numerous depressions and rises in it. The experimenters believed this resulted in a loss of ground-scattered photons near some of the detectors with a consequent $10 \%$ reduction in the exposure rate. ${ }^{29}$ The $\mathrm{MCNP}$ calculations of the exposure rate had a $4 \%-6 \%$ relative crror associated with thern. Given these experimental uncertainties, it is clear from the data that MCNP accurately predicts the skyshine benchmark experimental measurements.

\section{BENCHMARK PROBLEM FIVE - COBALT-60 AIR-OVER- GROUND PROBLEM}

\section{A. Problem History and Description}

The ${ }^{60} \mathrm{Co}$ air-over-ground problem has been investigated many times in the past thirty years because of its importance in military and civil defense studies. In this problem, an infinite horizontal plane separates an infinite soil medium from an infinite air medium. ${ }^{30}{ }^{60} \mathrm{Co}$ is then spread uniformly upon the surface of the ground. The radiation dose absorbed in air three feet above the ground is then calculated. This problem simulates the radiation environment in an open field covered by fallout from a nuclear weapon.

Experimental measurements of the dose above a fallout field have actually been made in several nuclear weapons tests. ${ }^{31}$ However, difticultı.s with making dose measurements involving real fallout led researchers to treat the air-over-ground problem as the single-isotope infinite media problem described above. Between 1957 and 1968, the radiation dose in this fallout problem was either measured or

\footnotetext{
28 Ibid, p. 415.

29 Ibid, p. 415.

30 A. E. Profio, Shielding Benchmark Problems, Radiation Shielding Information Center, Oak Ridge, TN, ORNL-RSIC-25 [ANS-SD-9] (1969), p. 4.0-2.

31 Ibid, p. 4.0-2.
} 
computed by this approach in at least twelve studies. ${ }^{32}$ These studies are summarized in Table 5.1.

The experiments that attempted to measure the dose in the air-over-ground problem represented the uniform fallout field by an array of point isotope sources placed in an open field. The contributions from the point sources were then integrated to simulate a uniform radiation source. Ionization chambers or film packs placed above the ground amidst the array were used to measure the dose in these experiments. The studies that attempted to compute the dose above an isotope field utilized either moments methods or Monte Carlo techniques.

A variety of geometries were used in the moments method simulations of this problem. The most accurate of the moments method geometries modeled the air/ground semi-infinite media of the problem by a single-material infinite medium (water or air) split into two densities. The semi-infinite soil medium was represented by a semi-infinite material medium with the density of soil and the air medium by a scrni-infinite material medium with the density of air. The air/ground interface was therefore a planar density (not material) interface. ${ }^{33}$ The uniformly-spread isotope gamma source was represented by either a point source or an infinite plane source. This kind of geometry was necessary in the moments models because moments methods are limited to the use of one material as the scattering medium.

The Monte Carlo studies of the air-over-ground problem also used a variety of gcometries, ranging from the air/compressed-air geometry of the moments methods to the actual air/ground geometry of the problem. They represented the uniform fallout field with either a single point source or arrays of point sources distributed on the interface. At first, given the same amount of computer time and the same geometry model, the moments calculations were more accurate than the Monte Carlo calculations. ${ }^{34}$ As better and faster computers and computational techniques were developed, however, the accuracy of the Monte Carlo solutions eclipsed that of the moments simulations.

In 1987, Edward Lent and Thomas Wilcox at LLNL used the COG Monte Carlo code to simulate the air-over-ground problem. ${ }^{35}$ They used an air/soil geometry and calculated the dose buildup factor and angular kerma rate distribution at a

\footnotetext{
Ibid, P. 4.(1-14.

33 Ibid, p. 4.0-13

34 Ibid, p. 1.(1)-13.

35 Wilcox and Lent, (Ref. 8), p. 29
} 
TABLE 5.1

\section{SUMMARY OF AIR/GROUND RESEARCH (TAKEN, IN PART, FROM GARRETT)}

\begin{tabular}{|c|c|c|c|c|}
\hline INVESTIGATOR & METHOD & $Y E A R$ & BLLLDUP FACTOR & NOTES \\
\hline Berger & Moments & 1957 & 1.22 & $\begin{array}{l}\text { Point source in an infinite water geometry; } \\
\text { results integrated over source-detector } \\
\text { distance to obtain the buildup factor for } \\
\text { a plane source; function fitting was used } \\
\text { to reconstruct the flux density. }\end{array}$ \\
\hline Schlemm, et al. & Experiment & 1959 & $\begin{array}{l}1.15 \text { (6-M Tube) } \\
1.38 \text { (Film Pack) }\end{array}$ & $\begin{array}{l}2 \text { types of detectors were used to measure } \\
\text { ground level radiation at the apex of a } \\
\text { quadrant of point isotope sources. The } \\
\text { results were extrapolated to obtain the } \\
\text { total scattered exposure rate } 3 \mathrm{ft} \text {. above } \\
\text { an infinite isotope field. }\end{array}$ \\
\hline $\begin{array}{l}\text { Rexroad \& Schmoke, } \\
\text { Batter }\end{array}$ & Experiment & 1960 & 1.25 & $\begin{array}{l}\text { Ionization chamber measurements } 3 \mathrm{ft} \text {. } \\
\text { above a large array of }{ }^{60} \mathrm{Co} \text { point sources; } \\
\text { results extrapolated to a finite field }\end{array}$ \\
\hline Spencer & Moments & 1962 & 1.21 & $\begin{array}{l}\text { An infinite plane source in an infinite water } \\
\text { medium was used to calculate the upper } \\
\text { hemisphere contribution - this was assumed } \\
\text { to be equal to the lower hemisphere } \\
\text { scattered contribution; function fitting was } \\
\text { used to reconstruct the flux density. }\end{array}$ \\
\hline $\begin{array}{l}\text { Hubbell, Clark, } \\
\text { \& Buchanan }\end{array}$ & Moments & 1962 & 1.23 & $\begin{array}{l}\text { Infinite plane source in an infinite water } \\
\text { medium; polynomial expansion was used to } \\
\text { reconstruct the flux density }\end{array}$ \\
\hline $\begin{array}{l}\text { Plummer \& } \\
\text { Miller }\end{array}$ & Experiment & 1963 & 1.15 & $\begin{array}{l}\text { lonization chamber measurements of the } \\
\text { radiation from a }{ }^{60} \mathrm{Co} \text { capsule on the } \\
\text { ground were taken; the results were } \\
\text { extrapolated to an infinite uniform field. }\end{array}$ \\
\hline Marcum & Monte Carlo & 1965 & 1.16 & $\begin{array}{l}\text { Point source in an air/compressed air geometry; } \\
\text { results integrated over source-detector distance } \\
\text { to obtain dose builup factor; point sources were } \\
\text { arrayed } 2 \text { in. above the interface; } 12,750 \text { histories }\end{array}$ \\
\hline Berger & Moments & 1967 & 1.21 & $\begin{array}{l}\text { Infinite plane source in an infinite air } \\
\text { medium; polynomial expansion was used to } \\
\text { reconstruct the flux density. }\end{array}$ \\
\hline Berger & Monte Carlo & 1967 & 1.22 & $\begin{array}{l}\text { Point source in an infinite water medium - } \\
\text { results were integrated over the source-detector } \\
\text { distance; } 10,000 \text { histores. }\end{array}$ \\
\hline French & Monte Carlo & 1967 & 1.18 & $\begin{array}{l}\text { Randomly distributed point sources in an } \\
\text { air/ground geometry; COHORT code was used; } \\
7,000 \text { histories. }\end{array}$ \\
\hline Garrett & Monte Carlo & 1968 & 1.20 & $\begin{array}{l}\text { Selected distribution of point sources } \\
\text { in an air/compressed air geometry; L05 } \\
\text { code used, } 57,500 \text { histories. }\end{array}$ \\
\hline Kalos & Monte Carlo & 1968 & 1.20 & $\begin{array}{l}\text { Distribution of point sources in an } \\
\text { air/ground geometry; GADJET adjoint } \\
\text { Monte Carlo code used. }\end{array}$ \\
\hline Wilcox ${ }^{11}$ & Monte Carlo & 1972 & $1.23 \pm .02$ & Air/ground geometry - MORSE-L code used \\
\hline Wilcox & Monte Carlo & 1987 & $1.18 \pm .02$ & Air/ground geomelry - COG code used \\
\hline Whalen, et al. & Monte Carlo & 1990 & $1.190 \pm .005$ & $\begin{array}{l}\text { Air/ground geometry - MCNP4 } \\
\text { used, } 1.50 \text { million histories. }\end{array}$ \\
\hline
\end{tabular}

11 Wilcox and Lent, (Ref. 8), p. 32. 
point three feet above the ground. The dose buildup factor is the ratio of the total absorbed dose to the absorbed dose from uncollided photons. The angular kerma rate is directly related to the angular absorbed dose rate. In the air-over-ground problem, the numerical difference between the kerma rate and absorbed dose rate in air is negligible, so the two are taken to be equivalent. ${ }^{36}$

MCNP has now been used to model this fallout problem and compute the dose buildup factor and angular kerma distribution around a point three fect above the ground. These results have been compared to the COG results as well as the experimental and computational data from the previous research conducted on this problem. This previous research was compiled by C. W. Garrett in 1968 . He submitted this compilation along with his own Monte Carlo work on the problem to the ANS Standards Committee Compilation of Shiclding Benchmark Problems.

\section{B. MCNP Problem Model}

1. Problem Geometry / Material Compositions. The first step to modeling the air-over-ground problem was setting up its basic gcometry (see Fig. 5.1). A 1$\mathrm{km}$ radius sphere was centered at the origin of a cartesian coordinate system and cut into two hemispheres by a plane coincident with the XY-plane. The upper $(Z>O)$ hemisphere was filled with air, and the lower hemisphere was filled with soil. The density and composition (by weight fraction) of the air and soil were taken to be:

\begin{tabular}{llll} 
Air $: p=\underline{0.00120 \mathrm{gm} / \mathrm{cm}^{2}}$ & & \multicolumn{2}{c}{ Weight Fraction } \\
& nitrogen & $:$ & 0.7818 \\
oxygen & $:$ & 0.2097 \\
& argon & $:$ & 0.0085 \\
Soil $: \mathrm{p}=1.13 \mathrm{gm} / \mathrm{cm}^{3}$ & & & Weight Fraction \\
& oxygen & $:$ & 0.34 \\
& sodium & $:$ & 0.01 \\
& magnesium $:$ & 0.10 \\
& aluminum & $:$ & 0.03 \\
& silicon & $:$ & 0.18 \\
& sulfur & $:$ & 0.03 \\
& calcium & $:$ & 0.01 \\
& iron & $:$ & 0.29 \\
& nickel & $:$ & 0.01
\end{tabular}




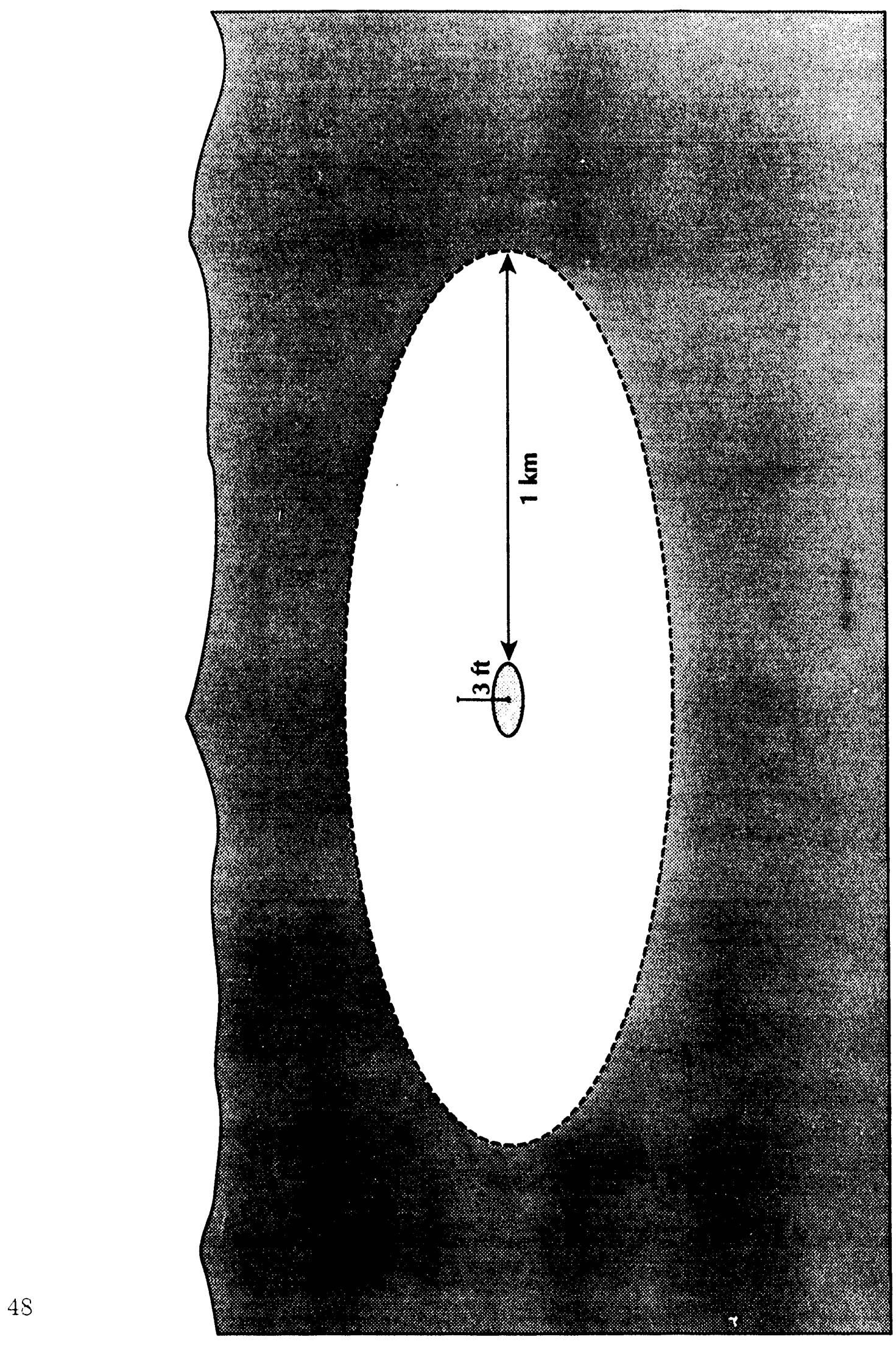

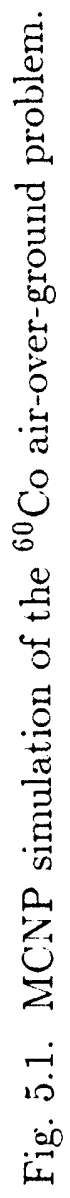


This is the composition of Nevada Test Site soil and air used in many Los Alamos calculations.

Three additional planes parallel to the XY-plane were then placed 6, 12, and $18 \mathrm{~cm}$ below it. This spacing was chosen because $6 \mathrm{~cm}$ is approximately one mean free pathlength (MFP) for a $1.33 \mathrm{MeV}$ photon in the soil. The XY-plane was the air/ground interface. A 1-km boundary was chosen for this problem model because $1000 \mathrm{~m}$ is approximately ten MFP for $1.33 \mathrm{MeV}$ gamma photons in air. MCNP weight window calculations indicated that photons backscattered to the origin from beyond this distance would make a negligible difference to the dose rates measured there.

Also, even though the entire lower hemisphere of soil was included in the problem, only the soil down to an $18-\mathrm{cm}$ depth contributed measurable photon backscatter to the origin. The $18-\mathrm{cm}$ plane beneath the soil constituted an "effective" lower boundary for the problem because the soil bencath it made only a negligible contribution to dose rates near the origin. After the problem geometry and material compositions were chosen, the geometry was further subdivided into cells.

2. Cell Subdivision. Adding fourteen concentric spheres centered at the origin to the problem geometry was the first step to partitioning the problem into cells. The air hemisphere was then divided into one hemispherical cell centered at the origin and fourteen hemispherical shell cells radiating out from the origin (see Fig. 5.2). The hemispherical shell cells were each defined to be the region between two consecutive spheres above the XY-plane. Next, the fourteen spheres were used to partition the soil hemisphere into cells.

The soil cells were defined to be the volumes enclosed by the intersections between the fourteen concentric spheres, the three soil planes, the outermost boundary sphere, and the air/ground interface. The resultant soil cells were:

1. hemispherical shell cells bounded above by the $18-\mathrm{cm}$ soil plane

2. one hemispherical cell bounded above by the $18-\mathrm{cm}$ soil plane and centered beneath the origin

3. three disk cells centered on the $\mathrm{z}$-axis between the air/ground interface above and the hemisphere cell below

4. three layers of flat concentric ring cells radiating out from the disk cells toward the problem boundary. 


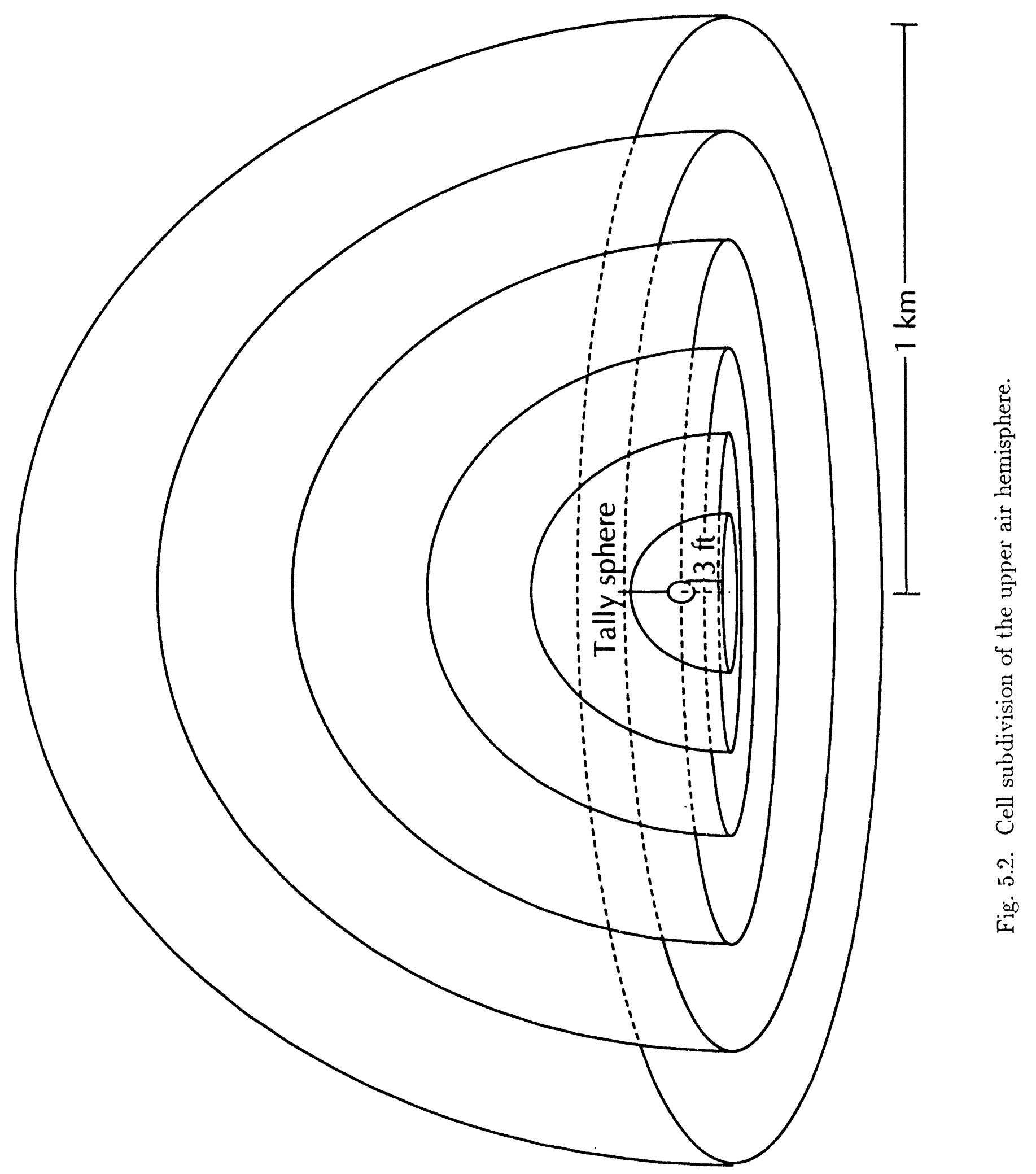


The cell subdivision is pictured in Fig. 5.3. One last spherical cell with a $0.5 \mathrm{~cm}$ radius was centered on the $z$-axis three feet $(91.44 \mathrm{~cm})$ above the ground. When the subdivision was complete, the problem geometry contained 142 cells. Complete details of this subdivision are found in the MCNP input file in Table A.6 in the Appendix.

3. Code Tallies. To obtain the MCNP estimate of the dose buildup factor, a point detector was placed three feet above the ground at the origin. The flux estimate of this tally was then modified by an FM card to obtain the energy deposition per unit volume in air per photon history $\left(\mathrm{MeV} / \mathrm{cm}^{3}\right.$. history), which is the dose per unit history. The MCNP F5 detector tally automatically separates its estimate into uncollided photon flux and total photon flux. With both of these flux estimates modified to yield dose, the ratio of the total dose tally to the uncollided dose tally was taken to obtain the buildup factor.

An F1 current tally was next placed on a $0.5-\mathrm{cm}$ sphere centered at the point detector in order to obtain the MCNP angular kerma rate over the small sphere. MCNP actually was used to calculate the angular dose rate in air, since kerma rates and dose rates are virtually identical there. A C1 card was then used to distribute the tallied current on the sphere surface into twenty cosine bins ranging from -1.0 to 1.0 . The angle was taken to be relative to the vector $(0,0,-1)$ from the detector point (see Fig. 5.4). The FT1 card with the FRV 001 option was used to specify this vector for the cosine binning.

The current in each cosine bin was further modified by an FM1 card to convert it into a dose rate and a CM1 card to normalize it per steradian. The FM1 card used to convert the current in each bin into a kerma rate (ergs/gm.sec) is:

$$
\text { FM1 } \quad \alpha \quad m-5 \quad-6
$$

where

$$
\alpha=\left(\frac{\text { atoms }}{\mathrm{cm} \cdot \mathrm{barn}}\right)\left(1.602 \times 10^{-6} \frac{\mathrm{erg}}{\mathrm{MeV}}\right)\left(\frac{1}{\rho}\right)\left(\frac{2}{\text { area of tally sphere }}\right)\left(\pi \times 10^{5} \frac{\text { source particles }}{\mathrm{sec}}\right)
$$

$\frac{\text { atoms }}{\text { cmbarn }}=$ atomic density of the material the tally surface is in (referred to as the atom density in the MCNP output file, it is calculated by the code)

$\rho=$ density of the tally sphere material, in $\mathrm{gm} / \mathrm{cm}^{3}$

$\mathrm{m}=$ material number, as specified in the input file. 

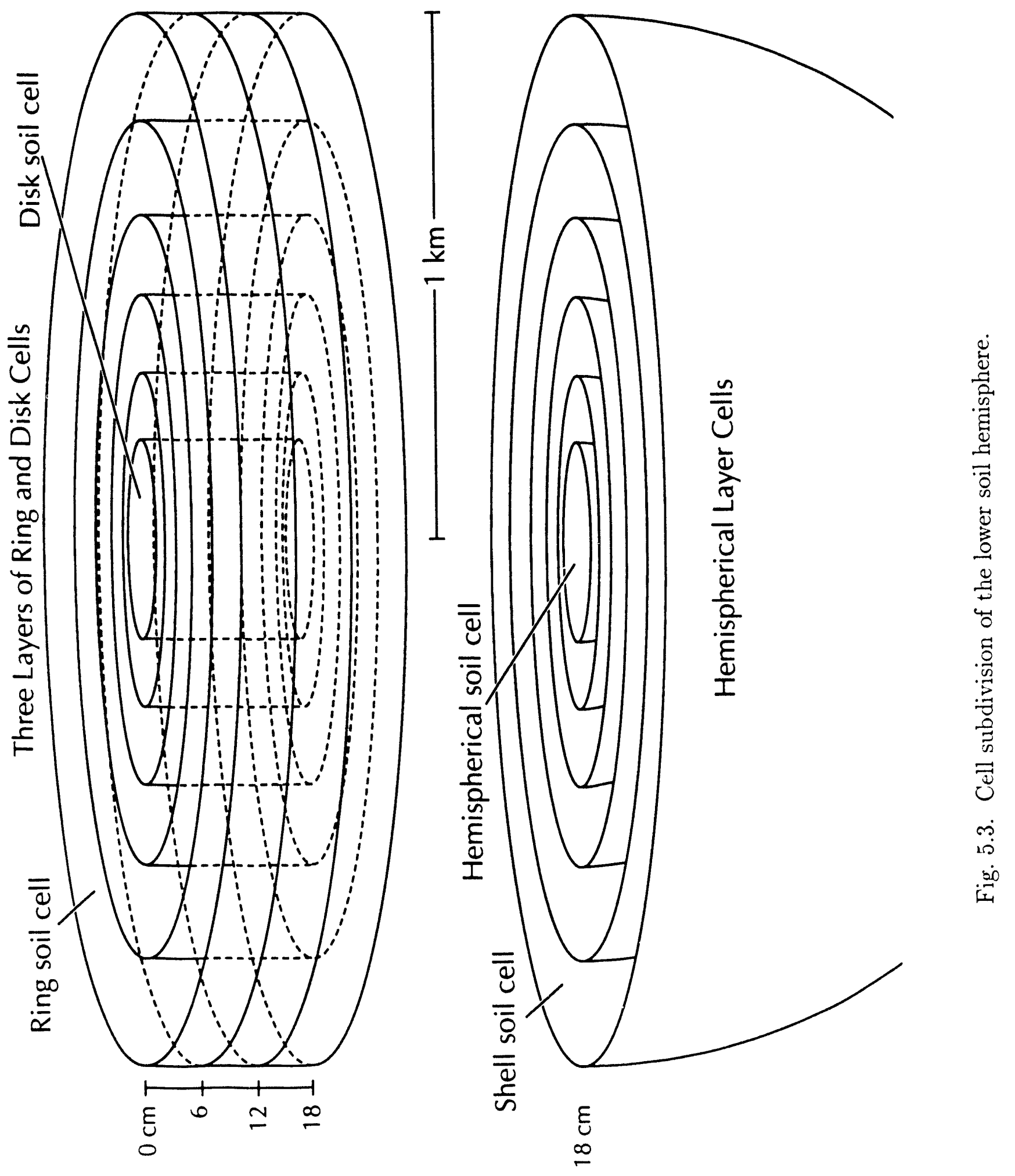


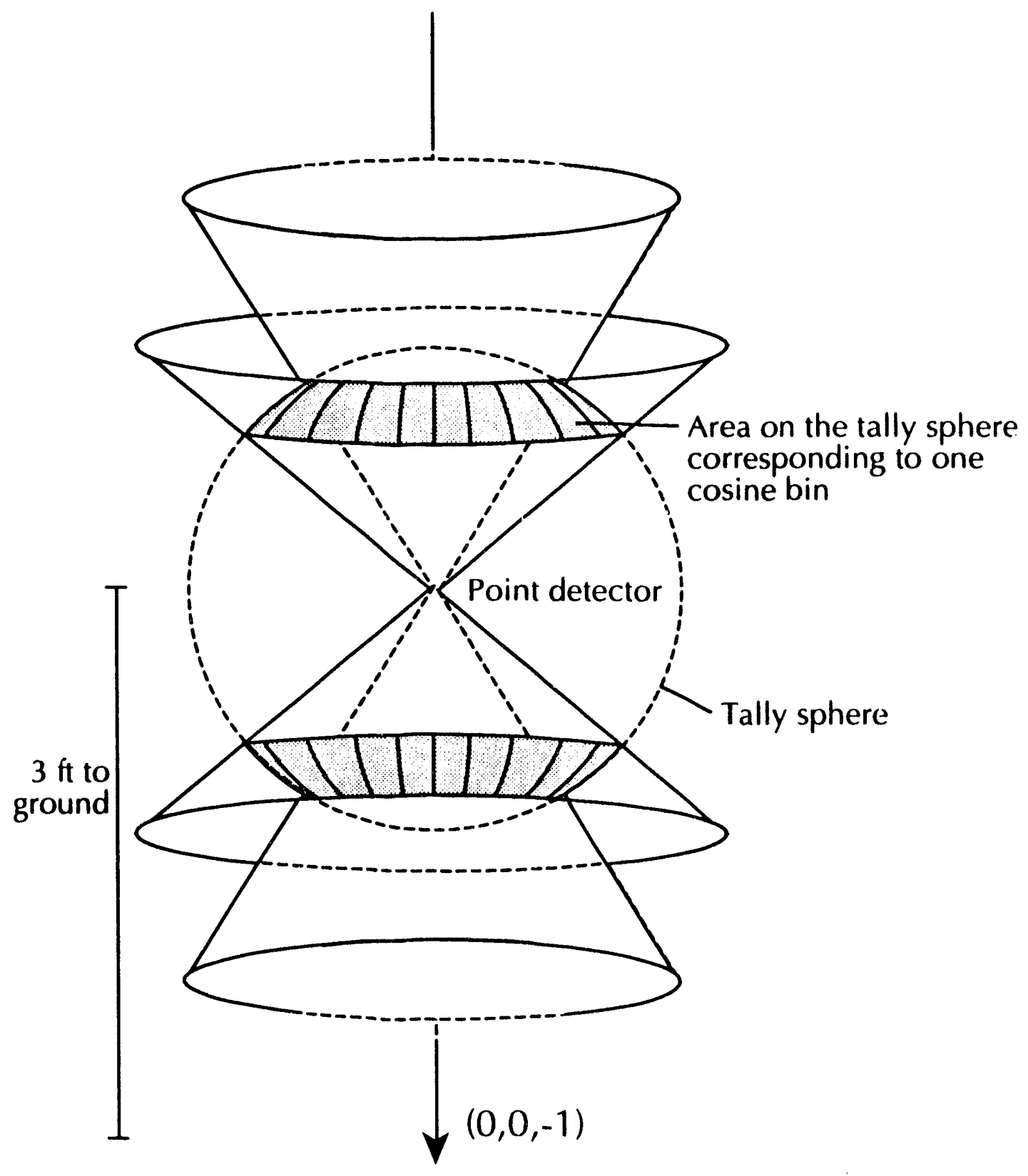

Fig. 5.4. The angular kerma rate tally over a sphere. 
To normalize the kerma rate of each cosine bin per steradian, it is multiplied by $1 / 2 \pi\left(\cos \Theta_{i+1}-\cos \Theta_{i}\right)$ with a CM1 card (see the CMn section of Chapter 3 in the MCNP manual). In Garrett's paper, $\cos \Theta_{i+1}-\cos \Theta_{i}=0.1$. Finally, the tally estimate was separated into uncollided photon kerma rate and scattered photon kerma rate by using the FT1 card INC option.

4. Isotropic Planar Gamma Source. After the code tallies were established, the MCNP geometry of the uniform ${ }^{60}$ Co fallout source was created. A $1-\mathrm{km}$ radius planar disk surface source was centered at the origin on the XY plane. Gamma photons at 1.17 and $1.33 \mathrm{MeV}$ were isotropically emitted with equal probability uniformly over the source area. This was accomplished by first partitioning the source disk into seventeen rings with an SI card. Next, a histogram source probability density was defined by an SP card. Each bin of the histogram assigned the corresponding source ring a probability density that increased linearly with the ring radius, ensuring that MCNP started particles uniformly from over the source area. Once the fallout gamma source geometry was chosen, several variance reduction techniques were employed to improve the problem convergence.

5. Variance Reduction. Although the geometry of this model may at first appear to be simple, the problem is far from trivial. There are great difficuities with sampling particles and following them through deep penetration into the air and soil. Particles started from the source close to the origin, as well as those started nearly one kilometer away near the boundary, must all be correctly sampled. Contributions to the problem tallies by particles that travel 2 MFP in soil $(\sim 12 \mathrm{~cm})$, as well as by those that travel 9 MFP in air $(\sim 900 \mathrm{~m})$, must be properly accounted for. To enable MCNP to converge to the problem solution in a reasonable amount of time, three variance reduction techniques were used.

First, importances were assigned to the cells. An initial set of importances was given to the cells, and the problem was then run with a weight-window generator. The cell weights computed by the generator provided insight into what cells in the problem were contributing to the tallies. From these weights, new import ances were assigned to the cells, and the problem was rerun several times thereafter. After each run. the importances were adjusted by trial and error to ensure that each cell's contribution to the tallies was appropriately sampled.

Sext. a DITRAN sphere was centered at the F5 point detector. Particles far from the origin had only a very small probability of being scattered toward the point 
detector. The DXTRAN sphere statistically concentrated particles at the point detector, thus ensuring that contributions from distant particles to the point detector were tallied with adequate statistics in a reasonable amount of time. Specifies of the DXTRAN sphere itself are found in the MCNP input file at the end of the chapter.

Finally, the source was biased so MCNP would better sample the areas of the source which contributed most to the tally scores. This biasing was accomplished with an SB histogram bias. The bias of each bin of the histogram corresponded to a source ring, and was chosen so that:

1. it would act in concert with the importances (not reverse their effect) and

2. the source was most heavily sampled near the origin.

The source particles nearest the origin contributed the most to the tallies; thereforc, the biases are greatest there. After these variance reduction techniques were incorporated into the model, the problem was run to obtain the MCNP predictions for the dose buildup and angular kerma distribution.

\section{Results and Discussion}

1. MCNP Results. MCNP computed a dose buildup factor of $1.190 \pm .005$, compared to $1.18 \pm 0.02$ for $\mathrm{COG}^{37}$ As seen in Table 5.1, previously calculated values of the dose buildup range from 1.16 to $1.23 \pm 0.02$, whereas the experimental values are between 1.15 and 1.38. The MCNP prediction of the dose buildup compares quite well to both the experimental and computed results. The MCNP results for the total and scattered angular kerma rates are found in Fig. 5.5 along with the corresponding COG data and experimental data.

In this graph, the angular kerma rate is plotted as a function of cosine relative to the vector $(0,0,-1)$ from the point detector. $\operatorname{Cos} \Theta<0$ denotes skyward angle bins, and $\cos \Theta>0$ denotes groundward angle bins. Since the source is spread upon the ground, there are no uncollided photons coming from the sky. Consequently, for $\cos \Theta<0$, only the scattered kerma rates are seen; whereas both scattered and total rates are seen for $\cos \Theta>0$.

The MCNP/COG agreement for the total angular kerma rates for $\cos \Theta>0$ is good. Their agreement for scattered kerma rates for all $\Theta$ is not as good. However, the uncertainty in the COG data is unknown. The large fluctuations in the COG

$3 \bar{i}$ Wilcox and Lent, (Ref. 8), p. 32. 


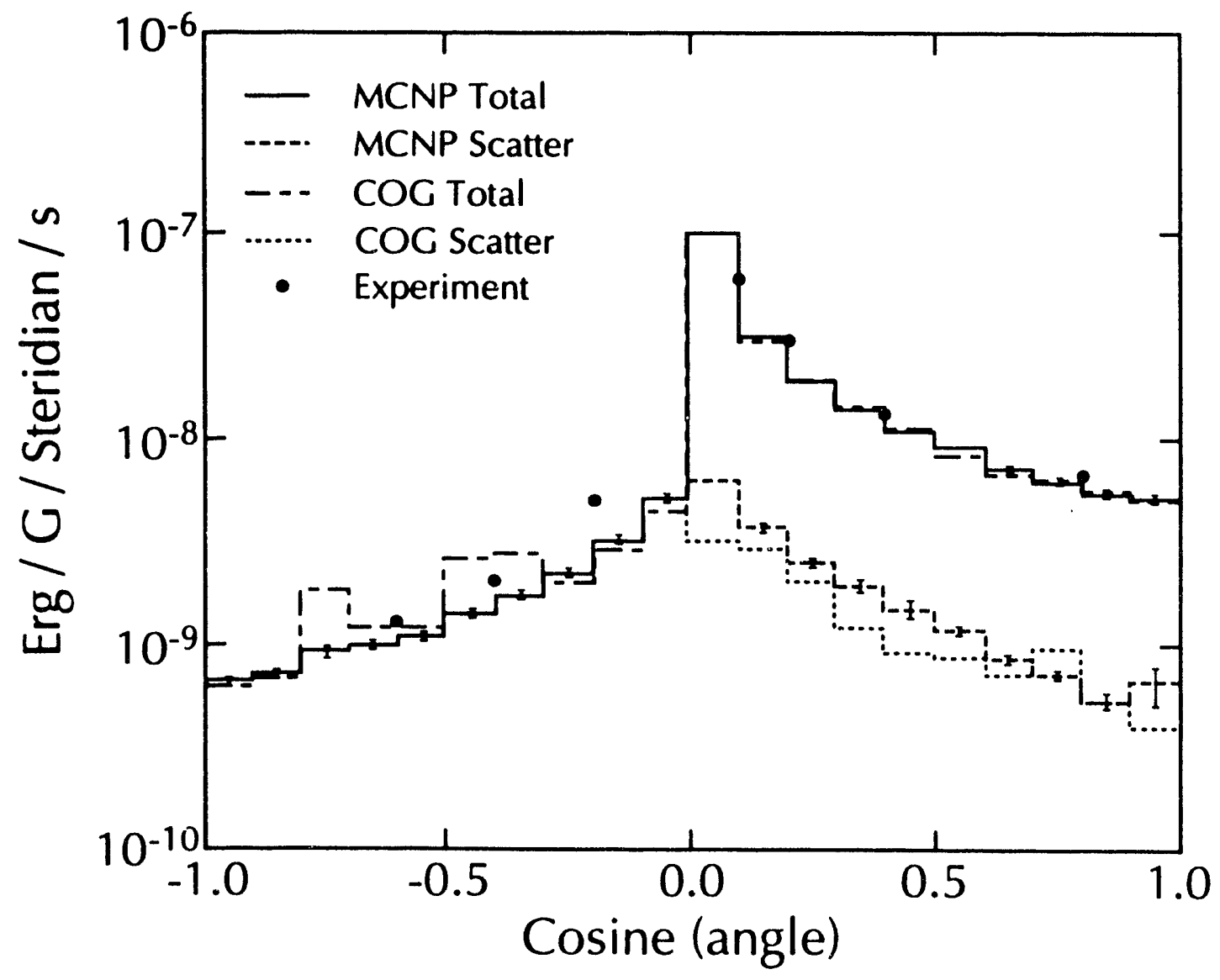

Fig. 5.5. Angular kerma rate distribution $3 \mathrm{ft}$. above ground. 
data for the scattered rates suggest that they lave a large relative error which could explain the discrepancy in agreement. The MCNP/experimental agreement in the total kerma rates is very good. MCNP generally agrees with the experimental scattered kerma rate data for $\cos \Theta<0$. There is a discrepancy between the two at $\cos \Theta=-0.2$, probably caused by a large experimental uncertainty there.

2. Experimental/Computational Error. In the ${ }^{60} \mathrm{Co}$ air-over-ground rescarch done by Garrett and earlier investigators, several sources of computational and experimental error are present. In the experiments listed in Table 5.1 (compiled by Garrett), point sources of isotopes were integrated to represent a uniform fallout field. Garrett estimated that this introduced an error of $\pm 5 \%$ in the experimental values of the dose buildup. ${ }^{38}$ Moments method calculations did not allow the proper problem gcometry to be represented, and it is not known exactly how much error this introduced into the moments results. Similarly, the Monte Carlo simulations either did not properly represent the problem geometry or the uniform fallout source. Garrett estimates that his own Monte Carlo computational error is no more than $10 \% .^{39}$ Given these experimental and computational uncertainties, MCNP was able to accurately model the radiation environment of the ${ }^{60} \mathrm{Co}$ air-over-ground problem.

\section{BENCHMARK PROBLEM SIX - HUPMOBILE TLD EXPERI- MENTS}

\section{A. Problem History and Description}

The Hupmobile thermoluminescent dosimeter (TLD) experiments were conducted by E. Goldberg et al. at Lawrence Berkeley Laboratory between 1967 and 1969. ${ }^{40,41}$ In two separate experiments, a single point source of gamma or x-rays was placed in air, one meter from one end of a toflon cylinder along its axis (see Fig.

\footnotetext{
38 Profio, (Ref. 7), p. 4.0-13.

39 Ibid, (Ref. 7), p. 4.0-13.

40 E. Goldberg, D. J. Groves, D. E. Jones, H. F. Luty, K. F. Petrock, G. A. Pohl, and D. H. White, Experiments to Test Validity of SORS-G Monte Carlo Code: I, Au-198 and Cs-137, Lawrence Livermore National Laboratory, Livermore, CA, UCID-121 (1967), p. 1.

41 E. Goldberg, D. J. Groves, D. E. Jones, H. F. Luty, K. F. Petrock, G. A. Pohl, D. II. White, and R. Worley, Experiments to Test Validity of SORS-G Monte Carlo Code, Lawrence Livermore National Laboratory, Livermore, CA, UCIR-368 (1969), p. 1.
} 
6.1). Seventeen LiF TLDs were imbedded inside the cylinder at specified intervals on its axis. One normalization TLD was also placed two meters from the end of the cylinder along its axis (one meter beyond the source).

The teflon cylinder and normalization TLD were then irradiated by the point source (usually for 5-7 days). Six different point source energies were studied:

$\left.\begin{array}{rl}{ }^{198} \mathrm{Au}: & 412 \mathrm{KeV} \\ { }^{137} \mathrm{Cs}: & 661 \mathrm{KeV} \\ & \\ { }^{60} \mathrm{Co}: & 1.33 / 1.17 \mathrm{MeV} \\ { }^{170} \mathrm{Tm}: & 84 \mathrm{KeV} \\ { }^{241} \mathrm{Am}: & 59.6 \mathrm{KeV} \\ \mathrm{Sm} \mathrm{K}_{\alpha} \text { x-rays: } & 39.9 \mathrm{KeV}\end{array}\right\}$ Experiment 1

The cylinder and reference TLDs registered the doses deposited in their locations in the teflon and air. The cylinder TLDs were then removed from the teflon. Their doses were read and divided by the normalization TLD air dose in order to make the experimental data independent of the source intensity.

The measured dose ratios were then compared to the values predicted for the experiment by the SORS-G Monte Carlo code. The Hupmobile experiments were carcfully designed to benchmark this then newly developed code. In 1987, Thomas Wilcox and Edward Lent used the Hupmobile experimental data to validate their COG Monte Carlo code. ${ }^{42}$ MCNP has now been used to model the Hupmobile experiments and predict their measured dose ratios. These computed relative doses compared favorably with the COG results and experimental data.

\section{B. MCNP Problem Model}

1. Experimental Arrangement. The layout of this experiment was fairly simple. A teflon cylinder was formed by stacking 11-inch diameter teflon disks into a 12-inch column. ${ }^{43}$ Seventeen LiF TLDs were implanted in the disks on their axes at specified depths. This teflon column was then loaded into an iron canister.

The canister was constructed from an iron cylinder and two steel disk endplates. ${ }^{44}$ The cylinder was $12 \mathrm{in}$. long, with an inner cliameter of $11 \mathrm{in}$. and a $1 / 4 \mathrm{in}$.

12 Wilcox and Lent, (Ref. 8), p. 17.

13 (ioldberg, et al. (Ref. 3), p. 1.

14 Ibid, p. 9. 


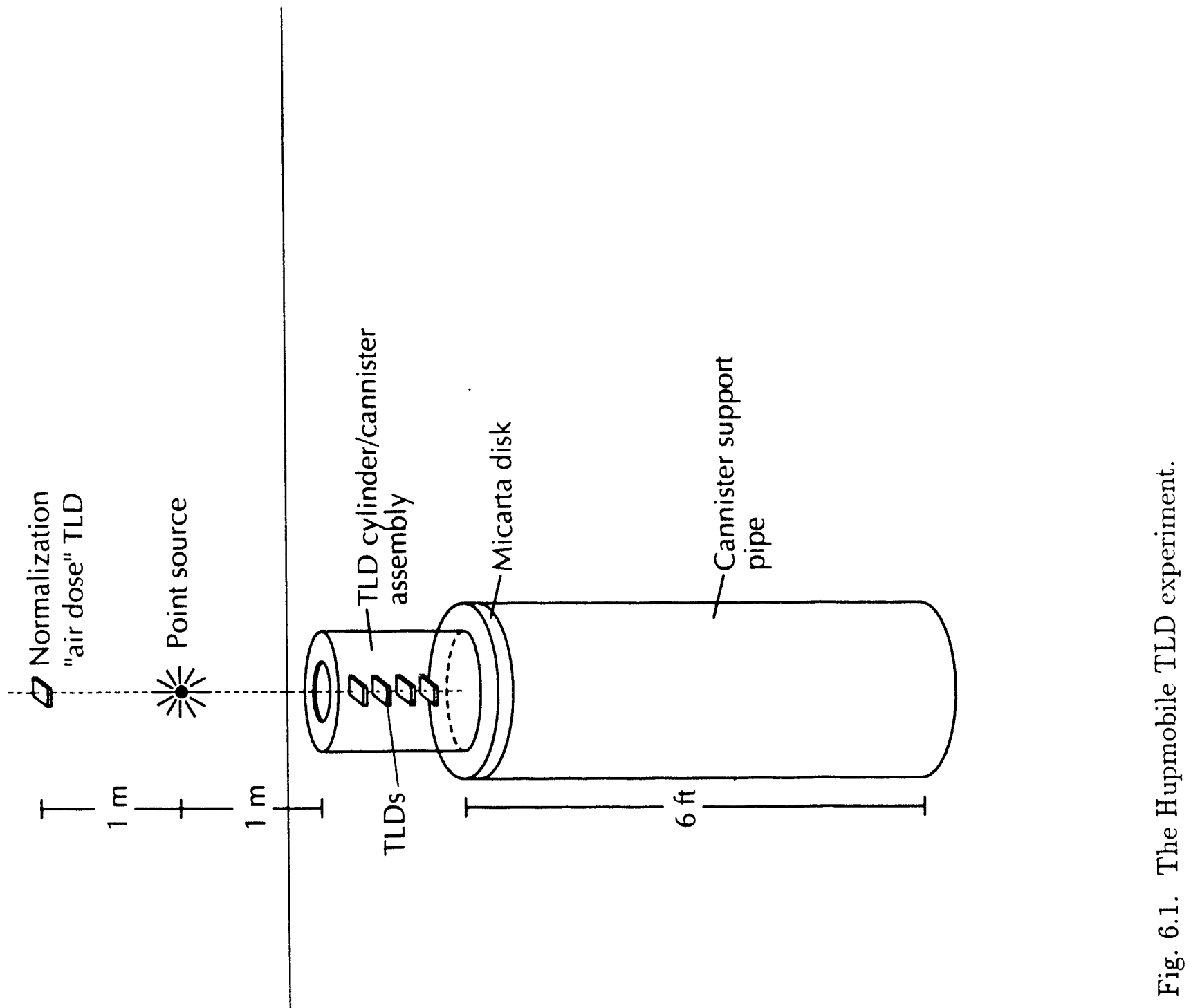


wall thickness. The upper endpiate (which would face the source) was an 11.5 in. diameter steel disk which was $3 / 8$ in. thick and had a 5.5 in diameter hole cut from its center. This hole was cut to allow some source photons to enter the teflon directly underneath the endcap. The rear endcap disk on the other end of the cylinder had an $11.5 \mathrm{in}$. diameter and was $1 / 4 \mathrm{in.}$ thick.

When the teflon/iron canister was assembled, it was placed upright upon a vertical stand rising from a concrete floor. ${ }^{45}$ This stand was a 12 in. diameter pipe that was $6 \mathrm{ft}$. tall and capped by a 1 in. thick micarta disk. Neither the wall thickness of the pipe nor the shape, diameter, nor composition of the micarta disk were specified. The rear endcap of the TLD cylinder rested flat upon the micarta disk of the stand.

Finally, the isotope point source was suspended in the middle of an aluminum ring onc meter above the upper cylinder face ${ }^{46}$ The ring size was not specified. Except that it had a concrete floor, the experimental area was not described. Information about wall and ceiling composition and location (or even whether this experiment was done indoors or outdoors) is therefore unknown.

2. Two MCNP Models. Two separate MCNP models were constructed to calculate the dose ratios of the Hupmobile experiment. The first model computed the radiation dose registered by the normalization TLD in the air. The second model calculated the doses measured by the TLDs in the teflon cylinder. The teflon doses of the second model were then divided by the air dose of the first to obtain the dose ratios. Two separate models were used rather than one because it was easier to optimize the efficiency of two problems than one combined problem.

3. Air Dose Geometry. A 118.11 in. radius cylinder was centered on the $\mathrm{z}$-axis of a coordinate system. This cylinder was parallel to the $\mathrm{z}$-axis and was capped by two planes at $z=-85.25 \mathrm{in}$. and $\mathrm{z}=137.80 \mathrm{in}$. This capped cylinder formed the problem boundary, and the region beyond it was made a void. A teflon cylinder $12 \mathrm{in}$. long and $11 \mathrm{in}$. in diameter was next centered on the z-axis. This cylinder was parallel to the z-axis with its upper endplate centered at the origin.

The teflon cylinder was then sheathed by a cylindrical iron shell $12 \mathrm{in}$. long with an inner diameter of $11 \mathrm{in}$. and a quarter-inch wall. The cylinder and shell were then capped by two iron disk endplates. The endplate at the $z=0$ end of the teflon

\footnotetext{
15 llid, p. 9.

16

Ibid, p. 9 .
} 
cylinder was $3 / 4 \mathrm{in}$. thick, $11.5 \mathrm{in}$. in diameter, and had a $5.5 \mathrm{in}$. hole cut from its center. The bottom endcap was $1 / 4 \mathrm{in}$. thick with an $11.5 \mathrm{in}$. diameter.

The support stand for the canister was then placed between the bottom face of the lower cylinder endcap and the floor (which was the $z=-85.25 \mathrm{in}$. boundary plane). The support stand was formed by a cylindrical iron pipe topped with a micarta disk. The pipe was $6 \mathrm{ft}$. long, with an outer diameter of twelve inches and a $1 / 5 \mathrm{in}$. wall. The micarta disk was $1 \mathrm{in}$. thick with a $12 \mathrm{in}$. diameter. It was given a teflon composition with a density of $1.491 \mathrm{~g} / \mathrm{cm}^{3}$. The hollow space inside the pipe was filled with air, as was the space cut out by the hole in the upper cylinder endplate. The region outside the cylinder and stand assembly (but within the problem boundary) was also filled with air. After this air dose geometry was constructed, it was subdivided into cells.

The teflon cylinder was defined by a single cell. The three iron canister components were each described by one cell. The two parts of the iron stand (iron pipe and micarta disk) were each defined by one cell. The air region inside the iron pipe stand was made a cell as was the air disk in the upper cylinder endcap. Finally, the air region outside the canister/stand assembly was divided into six additional cells, as described in the air dose input file at the end of the chapter. There was a total of fourteen cells.

4. Source/Tallies. A point isotropic source of photons was placed on the zaxis one meter above the upper face of the teflon cylinder. The source had an energy corresponding to the Hupmobile case being studied. A ring detector tally was then ccntered on the $z$-axis one meter above the source to estimate the dose that would be registered by the normalization TLD in the experiment. The flux estimate of this ring tally was modified by an FM card to obtain dose (in $\mathrm{MeV} / \mathrm{cm}^{3} \cdot$ history). The input file for the air dose geometry is found in Table A.7 of the Appendix.

5. Teflon Dose Model. Only the teflon cylinder/iron canister assembly and the point source were included in the teflon dose problem geometry. The canister support stand was not included because it did not significantly affect the scattered doses inside the teflon cylinder. The stand was included in the air dose model because it did affect the normalization dose in air. The teflon cylinder, iron canister, and relative placement of the point source were defined in the same manner as in the air dose model.

One difference in the teflon dose geometry was that the teflon cylinder was centered at the origin parallel to the $\mathrm{x}$-axis. Also, the problem boundary was a 118.11 
in. sphere (not a cylinder) centered at the origin. As in the air dose geometry, the region outside the canister but inside the problem boundary was filled with air. The teflon dose geometry was then subdivided into cells.

6. Cell Subdivision. The teflon cylinder and iron canister were divided into many more cells in this model than in the air dose model so that MCNP could more easily track the highly-scattered photons inside the cylinder and its iron casing. The cylinder and casing were subdivided into disk and cylindrical ring cells (see Fig. 6.2). The air disk cut out from the center of the upper canister endplate also defined a cell. The air outside the cylinder but inside the problem boundary was cut into three cells as described in the input files in Tables A.8 and A.9 in the Appendix. There was a total of 31 cells.

7. Code Tallies. Seventeen point flux estimators were placed inside the teflon cylinder on its axis. They were positioned to be where the LiF TLDs were imbedded in the Hupmobile cylinder. Point detectors were used in the ${ }^{60} \mathrm{Co},{ }^{137} \mathrm{Cs},{ }^{198} \mathrm{Au}$, and ${ }^{170} \mathrm{Tm}$ source cases. Ring detectors were used in the ${ }^{241} \mathrm{Am}$ and $\mathrm{Sm} \mathrm{K}_{\alpha}$ source models. The low-energy photons from these last two sources scattered more in the tefion than the higher-energy photons of the other four sources. The ring detectors used with these two sources yielded accurate tally estimates more efficiently than point detectors would have. The flux estimates were converted by an FM card (with a teflon atom density) into doses. Variance reduction techniques were then used to optimize the problem efficiency.

8. Variance Reduction. Two variance reduction techniques were used in the teflon dose model. First, importances were assigned to the cells by trial and error, thus enabling MCNP to more effectively sample the cells' contribution to the tally estimates. Next, the point source was biased to start the majority of its particles into a cone subtended by the upper cylinder endcap.

Increasing the sampling of the source into this cone allowed MCNP to spend most of its time tracking source particles that contributed the most to the tally estimates, thus considerably reducing the computer time required for MCNP to converge to an accurate result for the cylinder doses. After the problem was run and these doses were calculated, they were divided by the air normalization dose to obtain the MCNP estimates for the dose ratios. 


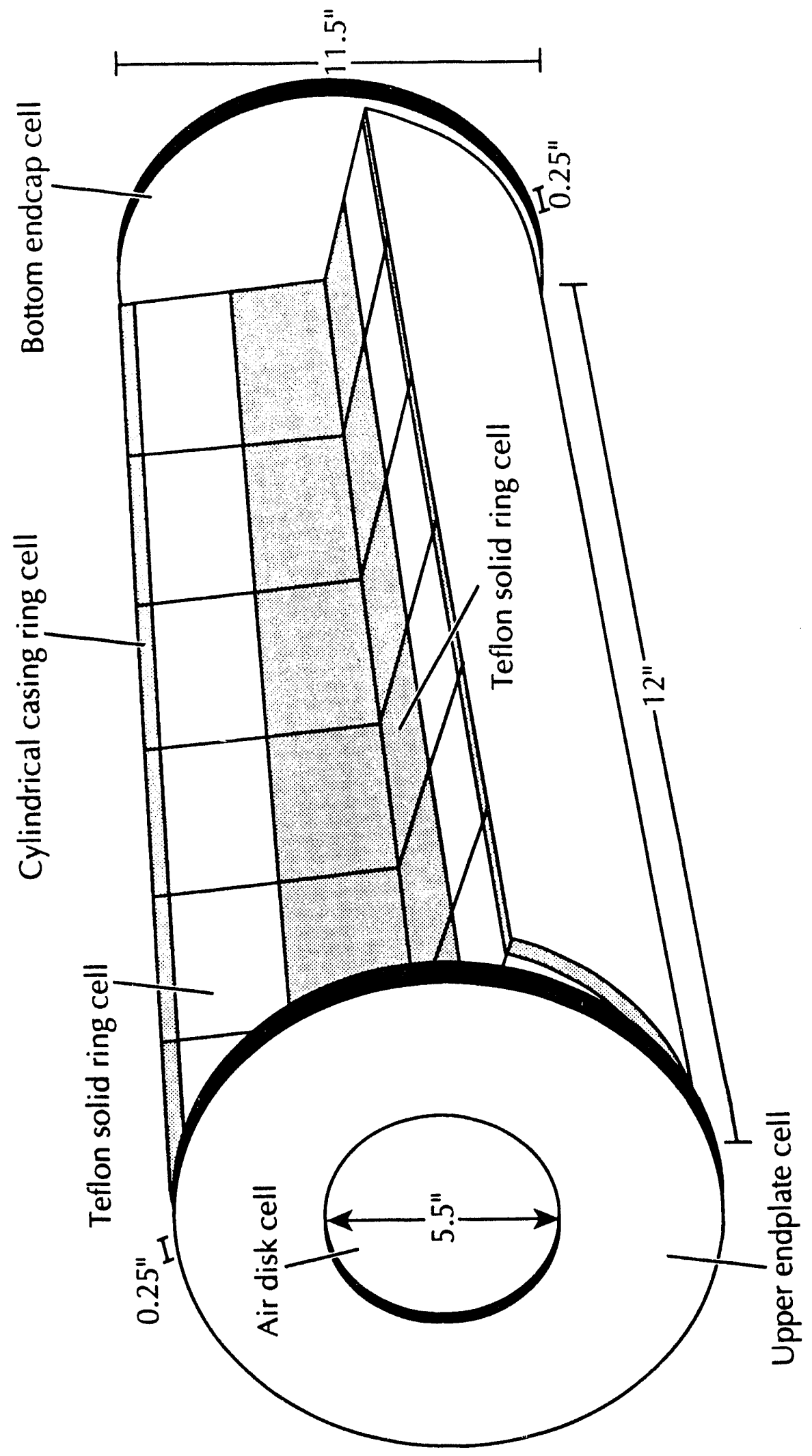

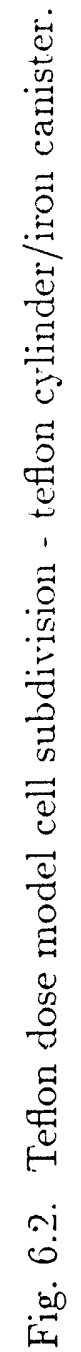




\section{B. Results and Discussions}

The experimental and calculated dose ratios for each source energy are plotted in Figs. 6.3 to 6.8. In the upper panel of each figure, the MCNP results appear with the experimental measurements ${ }^{47,48}$ and the COG data. ${ }^{49}$ The lower panel shows the difference between the MCNP and experimental relative doses, scaled to the total MCNP standard deviation:

$$
\frac{\mathrm{MCNP}-\mathrm{EXP}}{\sigma_{M C N P}}
$$

This expression will be bounded by \pm 1 when an MCNP dose ratio is within one standard deviation of the corresponding experimental ratio.

For ${ }^{60} \mathrm{Co},{ }^{137} \mathrm{Cs},{ }^{198} \mathrm{Au}$, and ${ }^{170} \mathrm{Tm}$, the agreement between MCNP, COG, and the experiment is good. For the ${ }^{241} \mathrm{Am}$ and $\mathrm{Sm} \mathrm{K}_{\alpha}$ sources, the agreement between MCNP and the experiment is good for detectors closer to the source. However, this agreement drops off after 6 in. into the teflon. The experimental uncertainty of the deeper detectors, while never explicitly stated, is probably fairly large. This could account for the discrepancy between MCNP and the experiment there. From these data, it is evident that MCNP accurately models the SORG-G Hupmobile TLD benchmark.

\section{SUMMARY}

Six families of photon transport benchmark problems have been run with MCNP4. These photon problem families were chosen as benchmarks because they were also used to validate the LLNL COG Monte Carlo code and because they represent a wide class of problems. MCNP accurately predicted the analytical or experimental results of all sixteen problems in these six families. These calculations demonstrate that MCNP can accurately simulate a wide class of photon transport problems.

An MCNP neutron benchmark document will soon follow.

\footnotetext{
47 Ibid, pp. 11-14.

18 Goldberg et al. (Ref. 4), pp. 8-18.

49 Wilcox and Lent, (Ref. 8), pp. 20-27.
} 

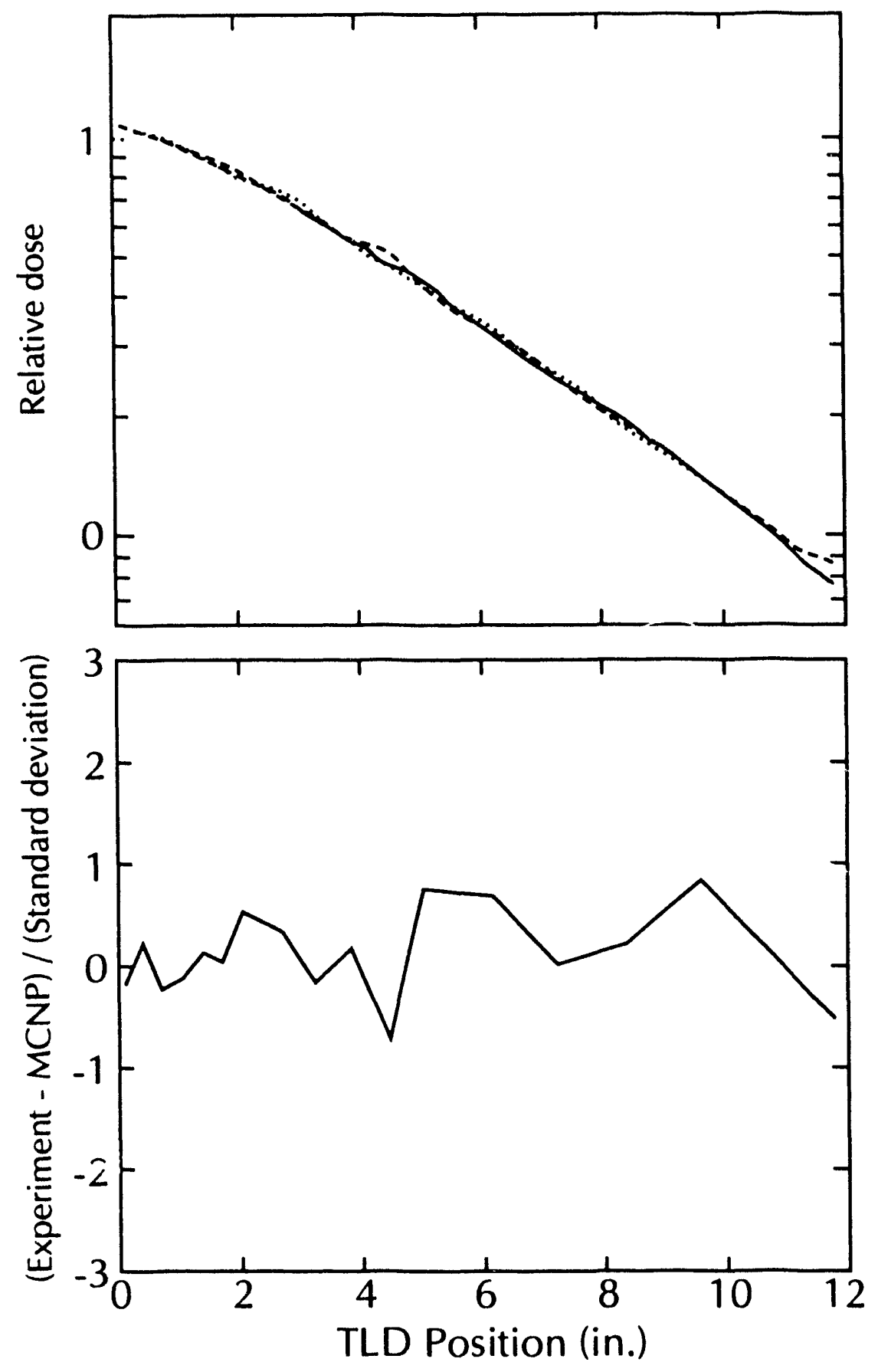

Fig. 6.3. For a ${ }^{60} \mathrm{Co}$ source, the upper panel shows relative dose for a Hupmobile experiment as measured (solid line) and as modeled by MCNP (long dash line) and by COG (short dash line). The lower panel shows the difference between MCNP and experiment, as scaled to the total standard deviation. 

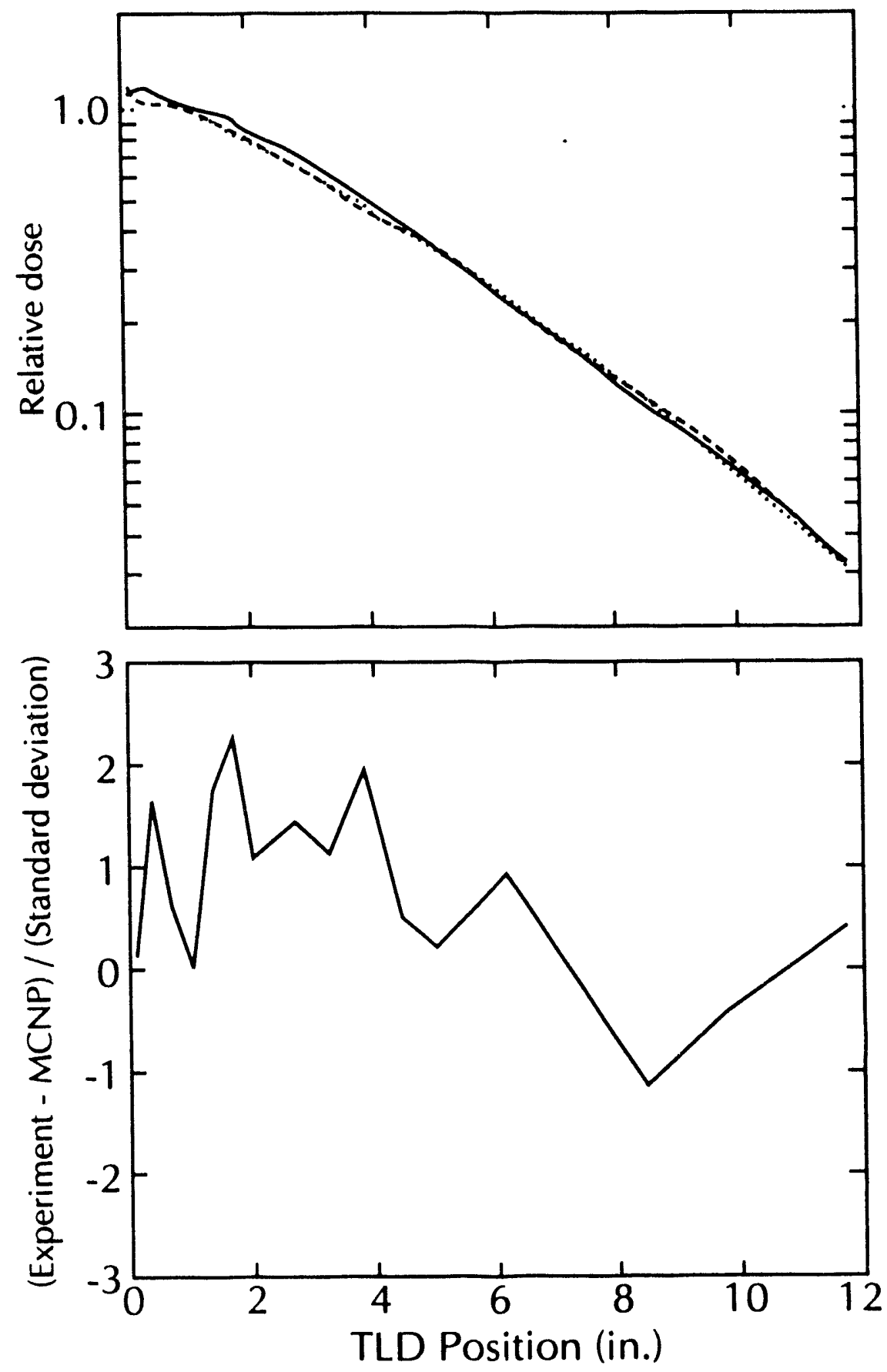

Fig. 6.4. For a ${ }^{137} \mathrm{C}$ s source, the upper panel shows relative dose for a $\mathrm{Hupmobile}$ experiment as measured (solid line) and as modeled by MCNP (long dash line) and by COG (short clash line). The lower panel shows the difference between MCNP and experiment, as scaled to the total standard deviation. 

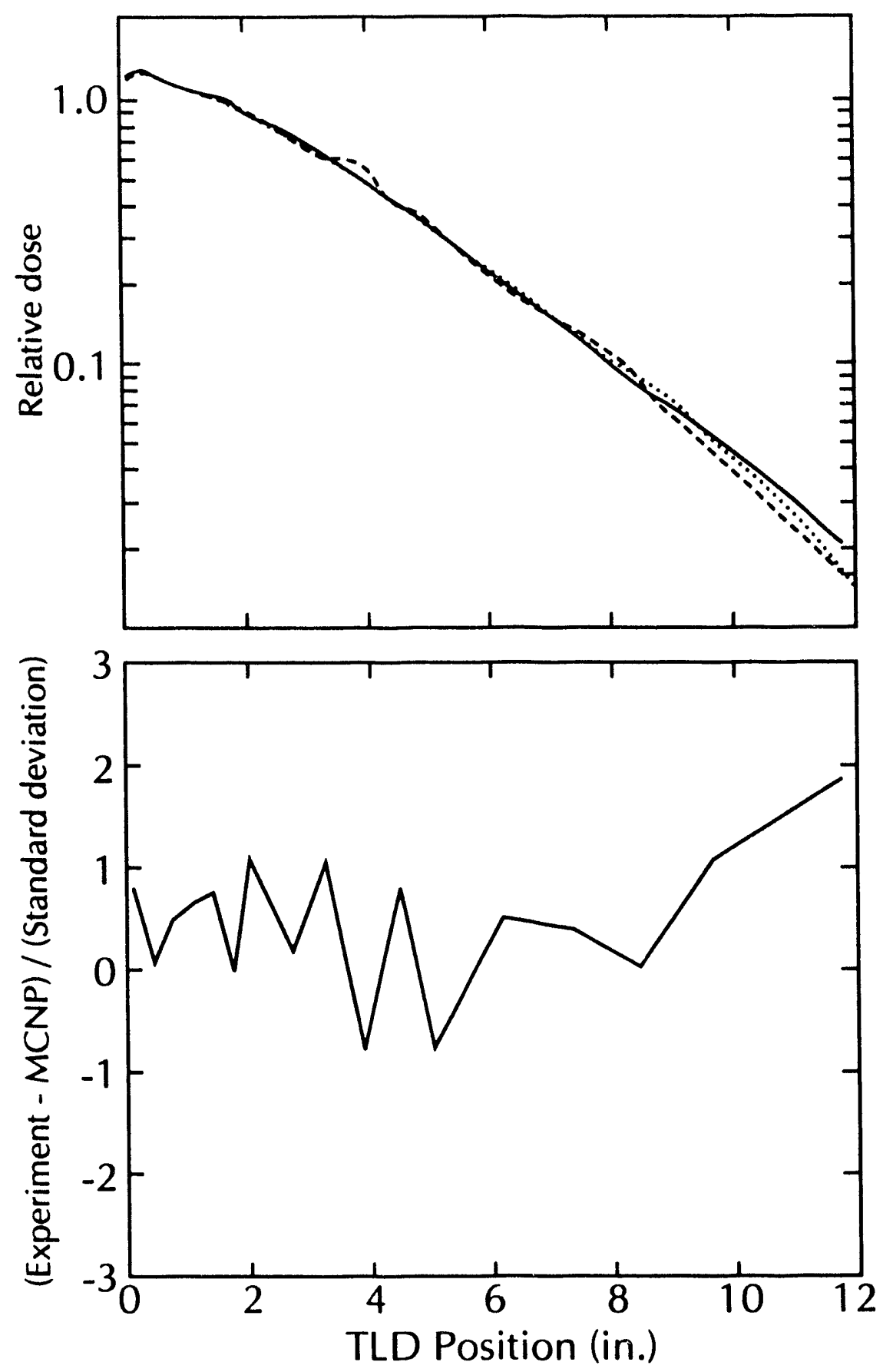

Fig. 6.5. For a ${ }^{198} \mathrm{Au}$ source, the upper panel shows relative dose for a Hupmobile experiment as measured (solid line) and as modeled by MCNP (long dash line) and by COG (short dash line). The lower panel shows the difference between MCNP and experiment, as scaled to the total standard deviation. 

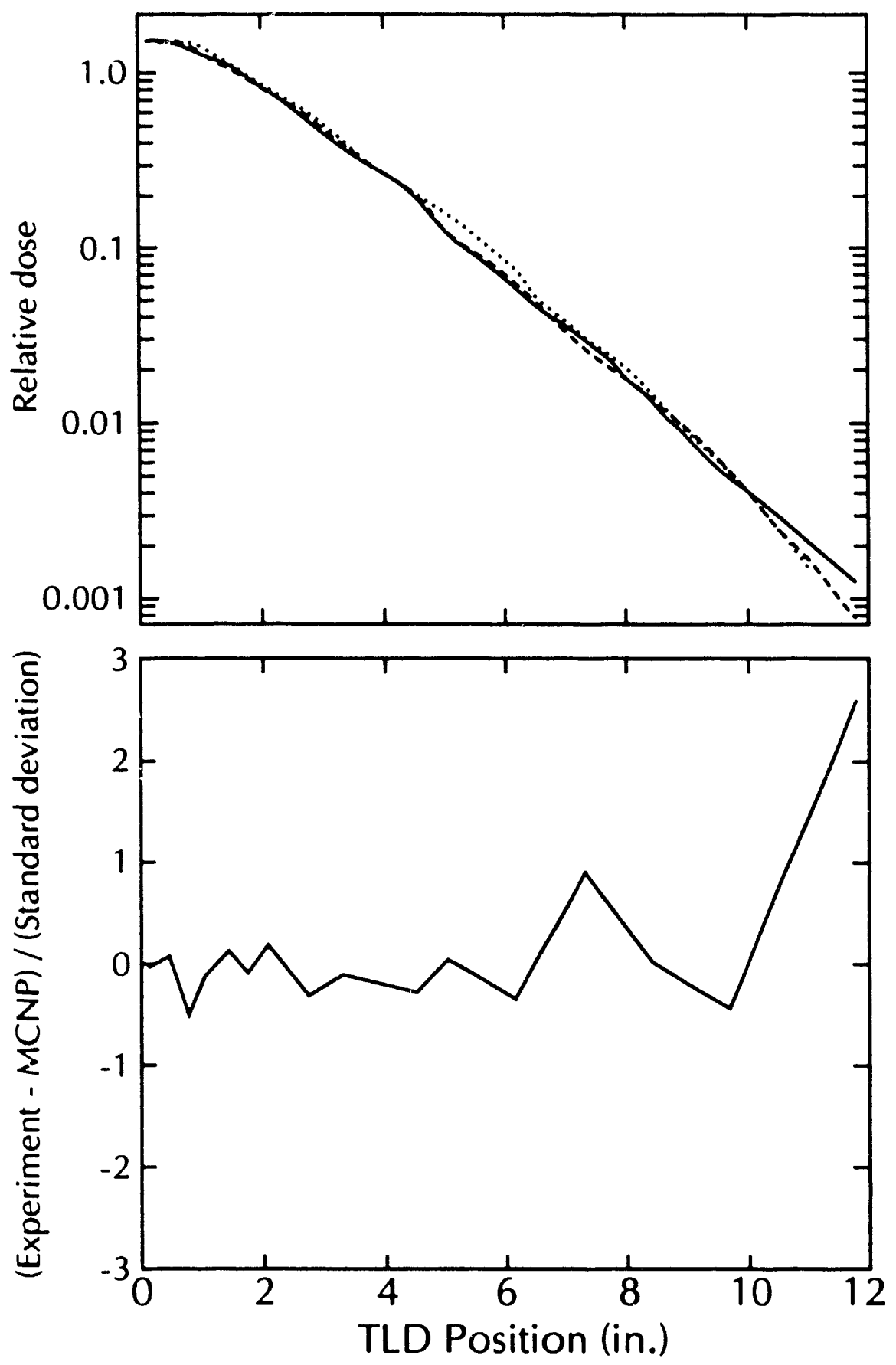

Fig. 6.6. For a ${ }^{170} \mathrm{Tm}$ source, the upper panel shows re' . tive dose for a Hupmobile experiment as measured (solid line) and as $\mathrm{m}^{\prime}$ )deled by MCNP (long dash line; and by COG (short dash line). The lower panel shows the difference between MCNP and experiment, as sialed to the total standard deviation. 

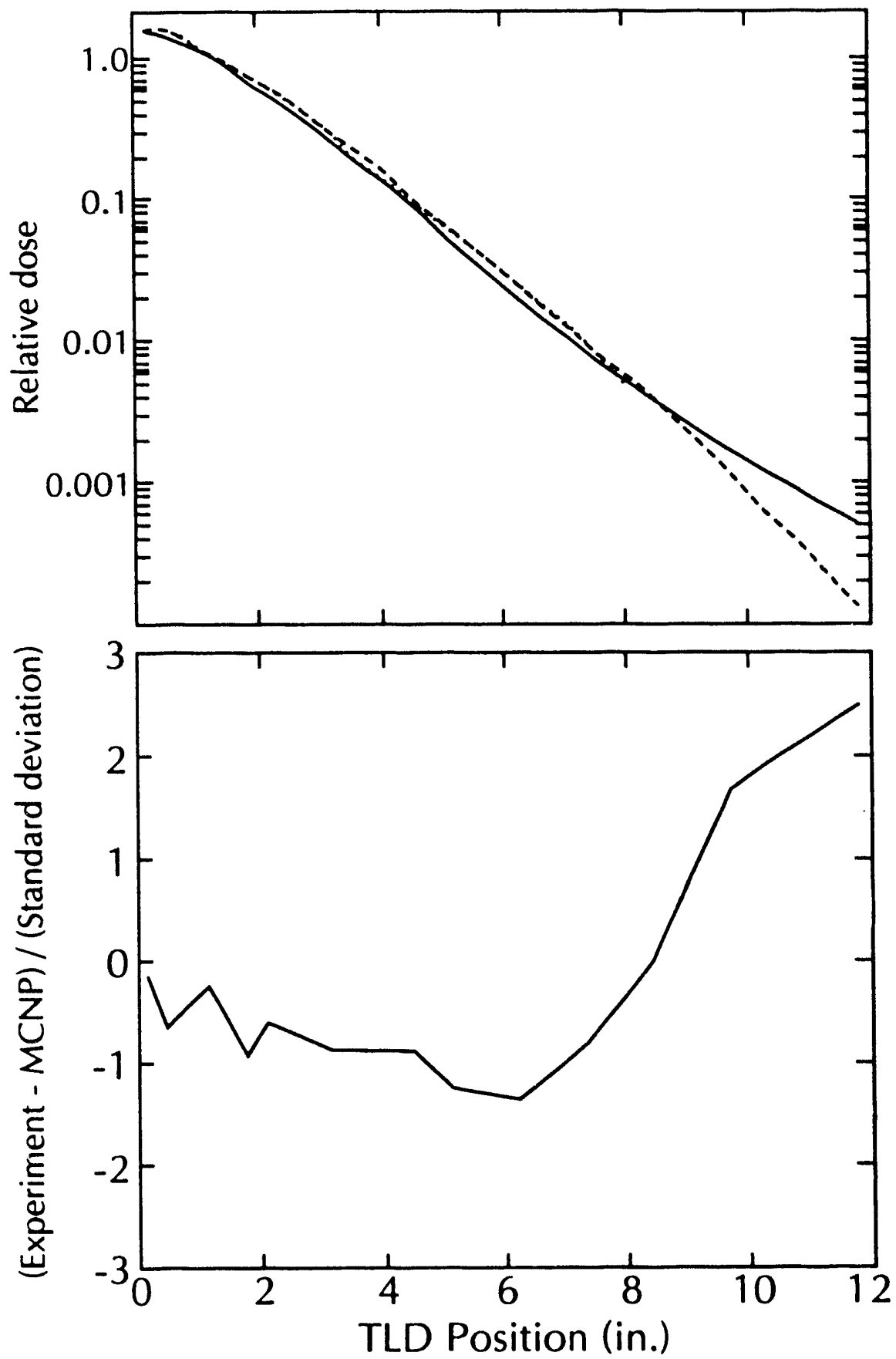

Fig. 6.7. For a ${ }^{241} \mathrm{Am}$ source, the upper panel shows relative dose for a Hupmobile experiment as measured (solid line) and as modeled by MCNP (long dash line) and by COG (short dash line). The lower panel shows the difference between MCNP and experiment, as scaled to the total standard deviation. 

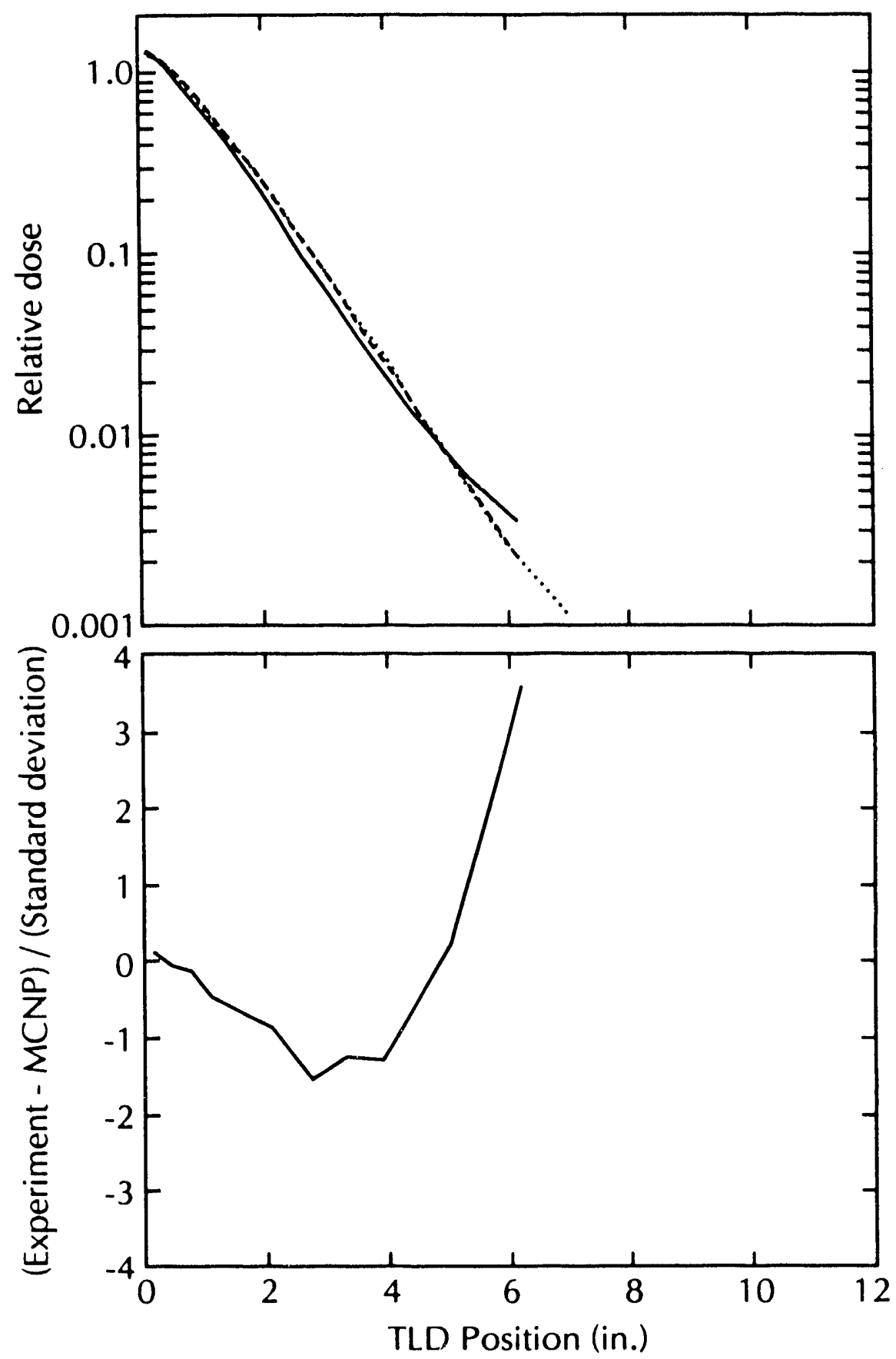

Fig. 6.8. For a $\mathrm{Sm}$ K-alpha source, the upper panel shows relative dose for a Hupmobile experiment as measured (solid line) and as modeled by MCNP (long dash line) and by COG (short dash line). The lower panel shows the difference between MC'NP and experiment, as scaled to the total standard deviation. 


\section{REFERENCES}

1. Judith F. Briesmeister, Editor, "MCNP - A General Monte Carlo Code for Neutron and Photon Transport, Version 3A," Los Alamos National Laboratory Report LA-7396-M, Rev. 2 (1986).

2. K. N. Case, F. de Hoffman, and G. Placzck, Introduction to the Theory of Neutron Diffusion, Vol. 1, U.S. Government Printing Office, Washington, D.C. (1953).

3. E. Goldberg, D. J. Groves, D. E. Jones, H. F. Luty, K. F. Petrock, G. A. Pohl, and D. H. White, Experiments to Test Validity of SORS-G Monte Carlo Code: I, Au-198, and Cs-137, Lawrence Livermore National Laboratory, Livermore, CA, UCID-121 (1967).

4. E. Goldberg, D. J. Groves, D. E. Jones, H. F. Luty, K. F. Petrock, G. A. Pohl, D. H. White, and R. Worley, Experiments to Test Validity of SORS-G Monte Carlo Code, Lawrence Livermore National Laboratory, Livermore, CA, UCIR368 (1969).

5. H. Goldstein and J. E. Wilkins, Jr., Calculations of the Penetration of Gamma Rays, Technical Information Service, Oak Ridge, TN, NYO-3075 (1954).

6. R. R. Nason, J. K. Shultis, R. E. Faw, and C. E. Clifford, "A Benchmark Gamma-Ray Skyshine Experiment," Nuclear Science and Engineering, 79, 404-416 (1981).

7. A. E. Profio, Shielding Benchmark Problems, Radiation Shielding Information Center, Oak Ridge, TN, ORNL-RSIC-25 [ANS-5D-9] (1969).

8. Thomas P. Wilcox, Jr. and Edward M. Lent, "COG - A Particle Transport Code Designed to Solve the Boltzmann Equation for Deep-Penetration (Shielding) Problems," Volume 4, "Benchmark Problems," Lawrence Livermore National Laboratory Report M-221-4 (12/2/88). 
APPENDIX:

INPUT FILES FOR BENCHMARKS 1-6

AND MCNP PATCH FILE 


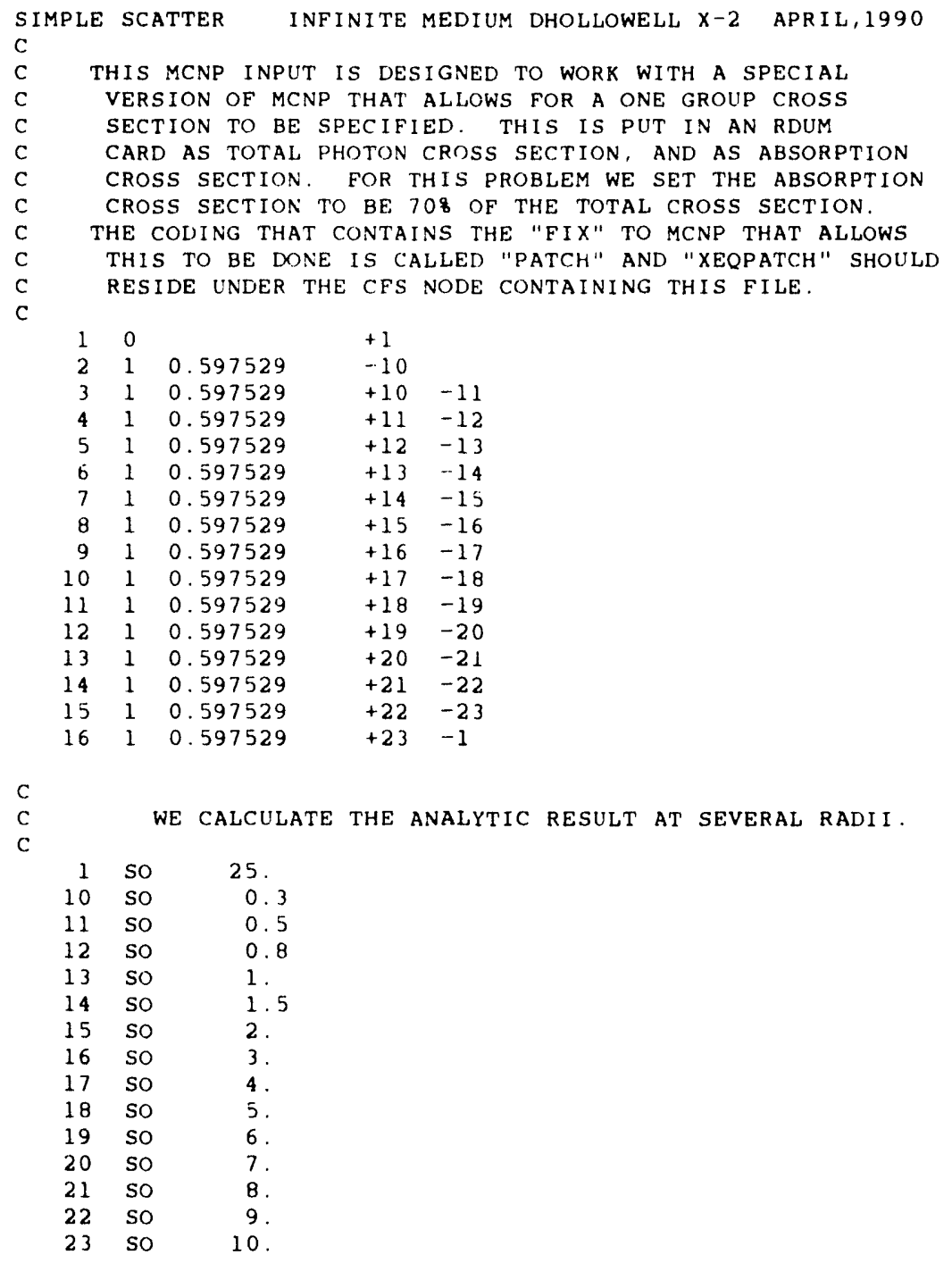

Table A.1: Input file for Benchmark One: $30 \%$ scattering case. The $0 \%$ and $90 \%$ scattering cases are done by modifying the cross sections on the RDUM card. 


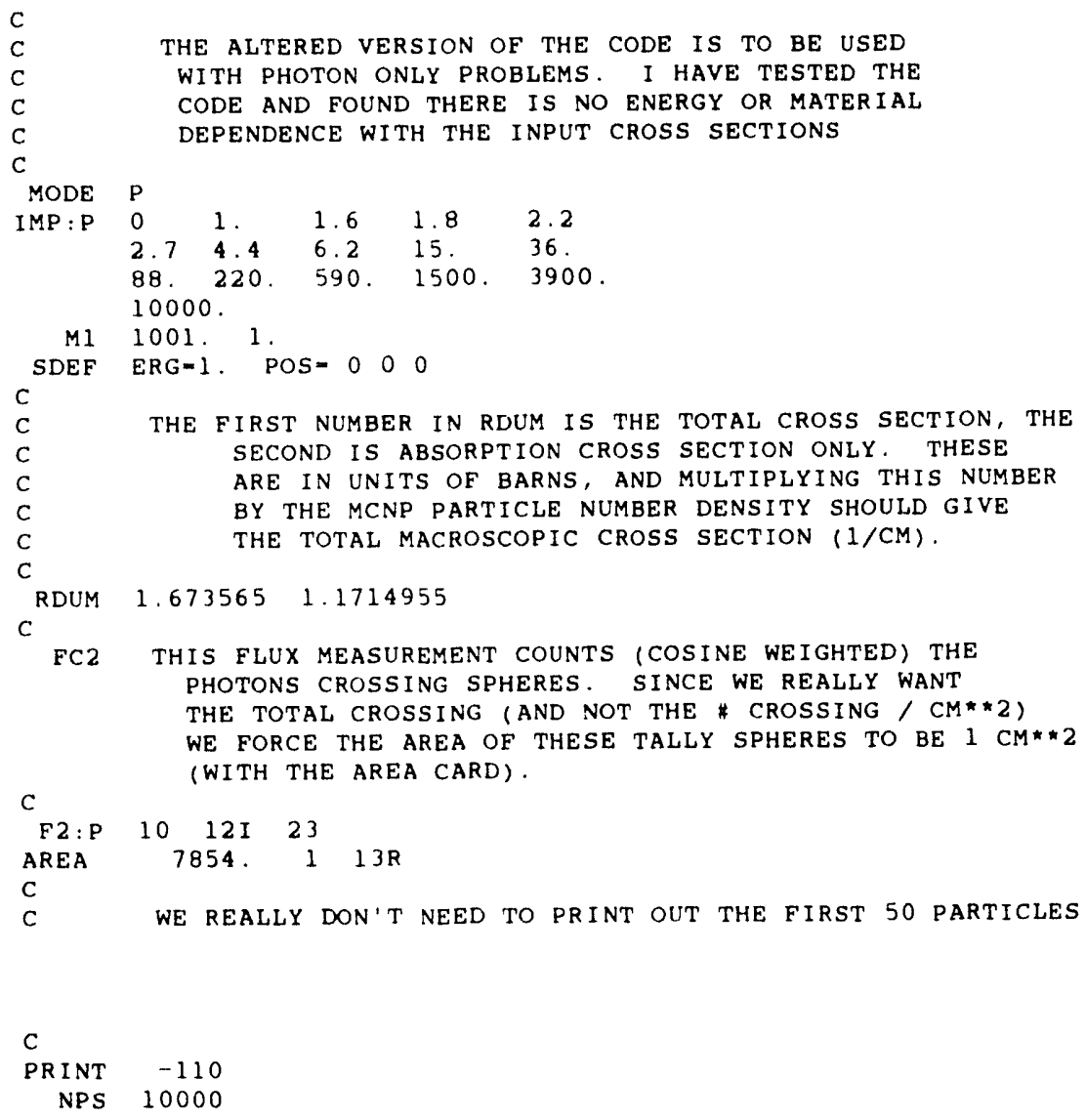

Table A.1: (cont) 


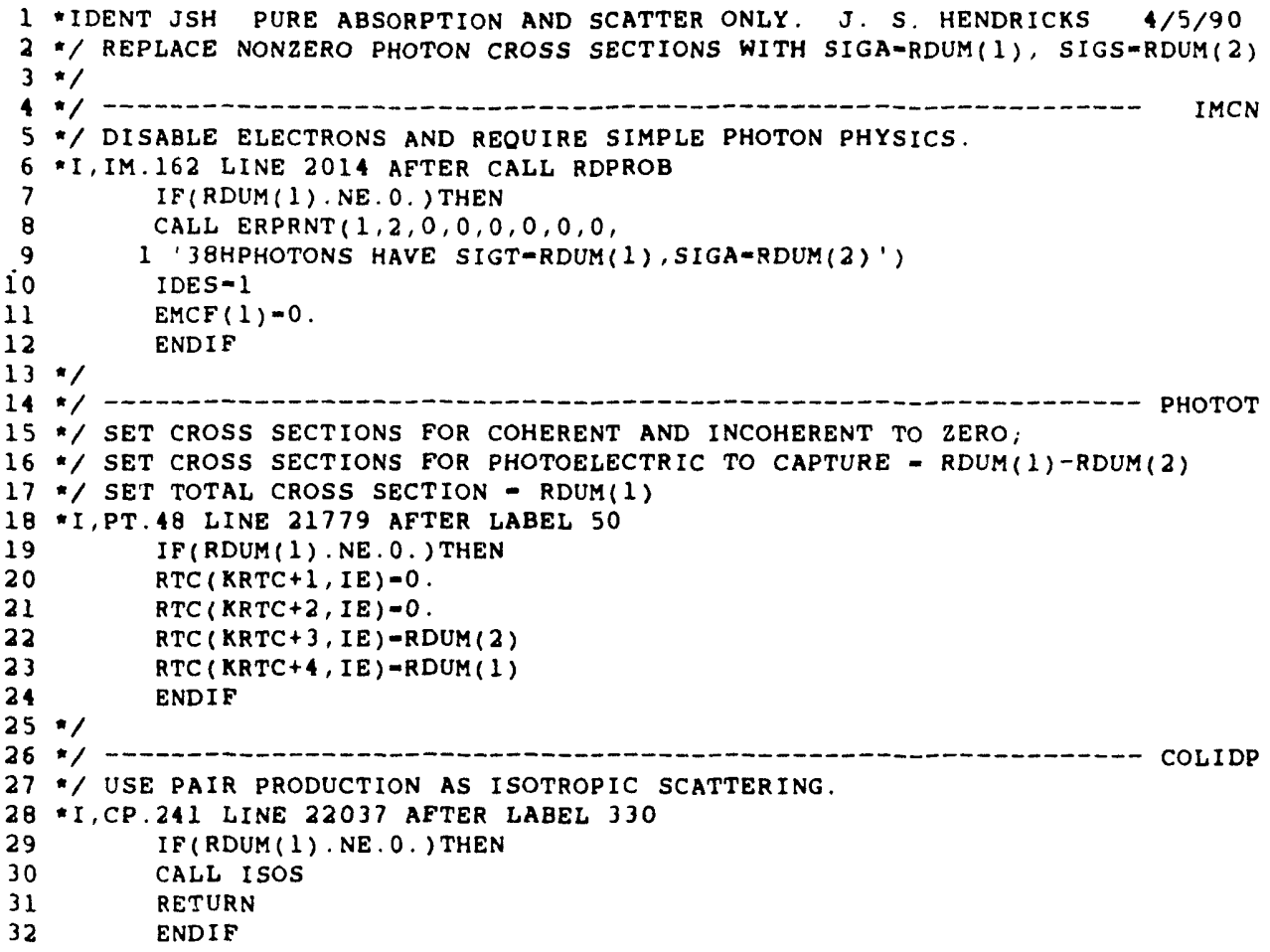

Table A.2: Patch file for Benchmark Problems One and Two. Modification to MCNP4 to treat photon absorption as photoelectric capture and model isotropic scatter as photoelectric fluorescence. 


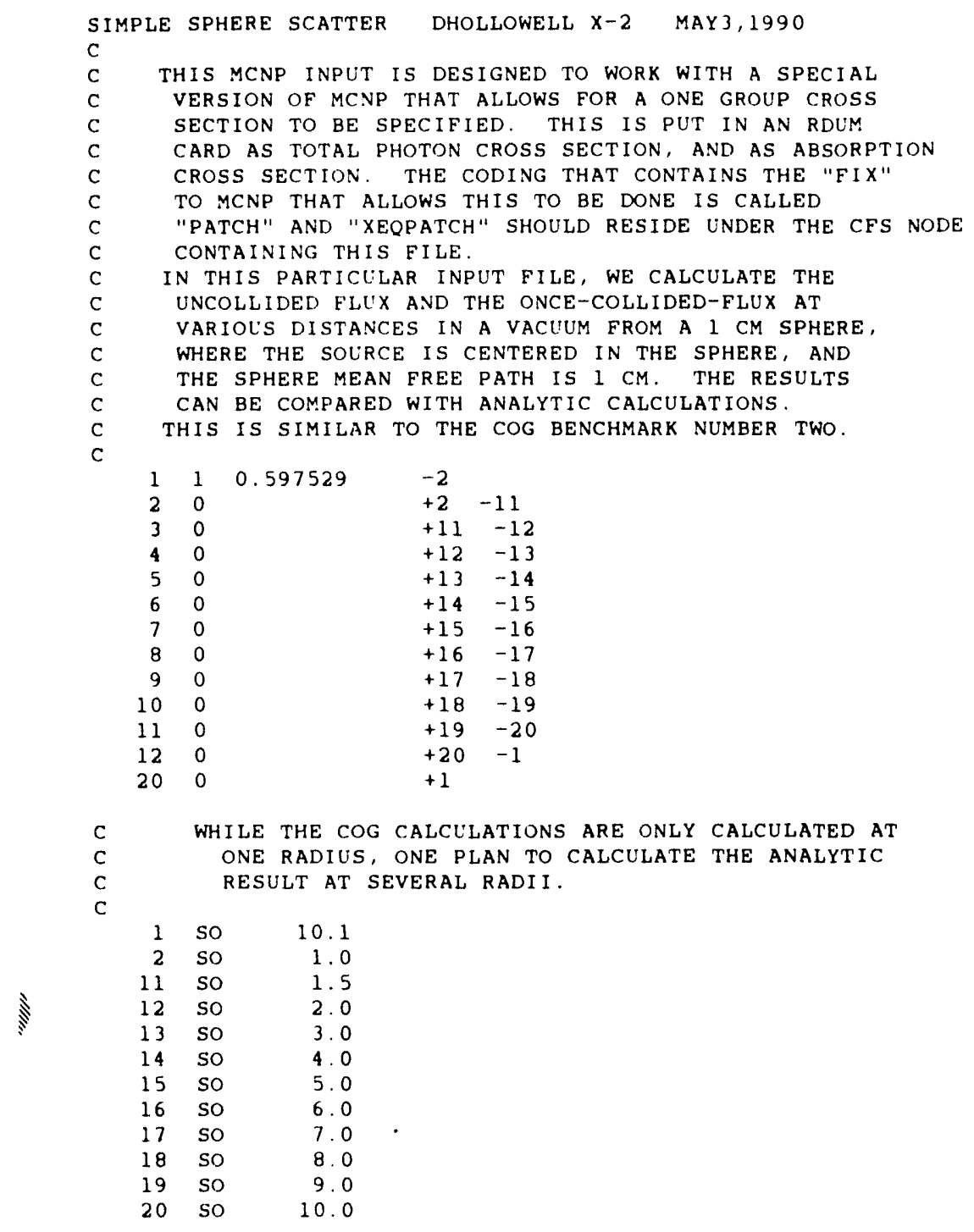

Table A.3: Input file for Benchmark Two: single sphere scatterer. 


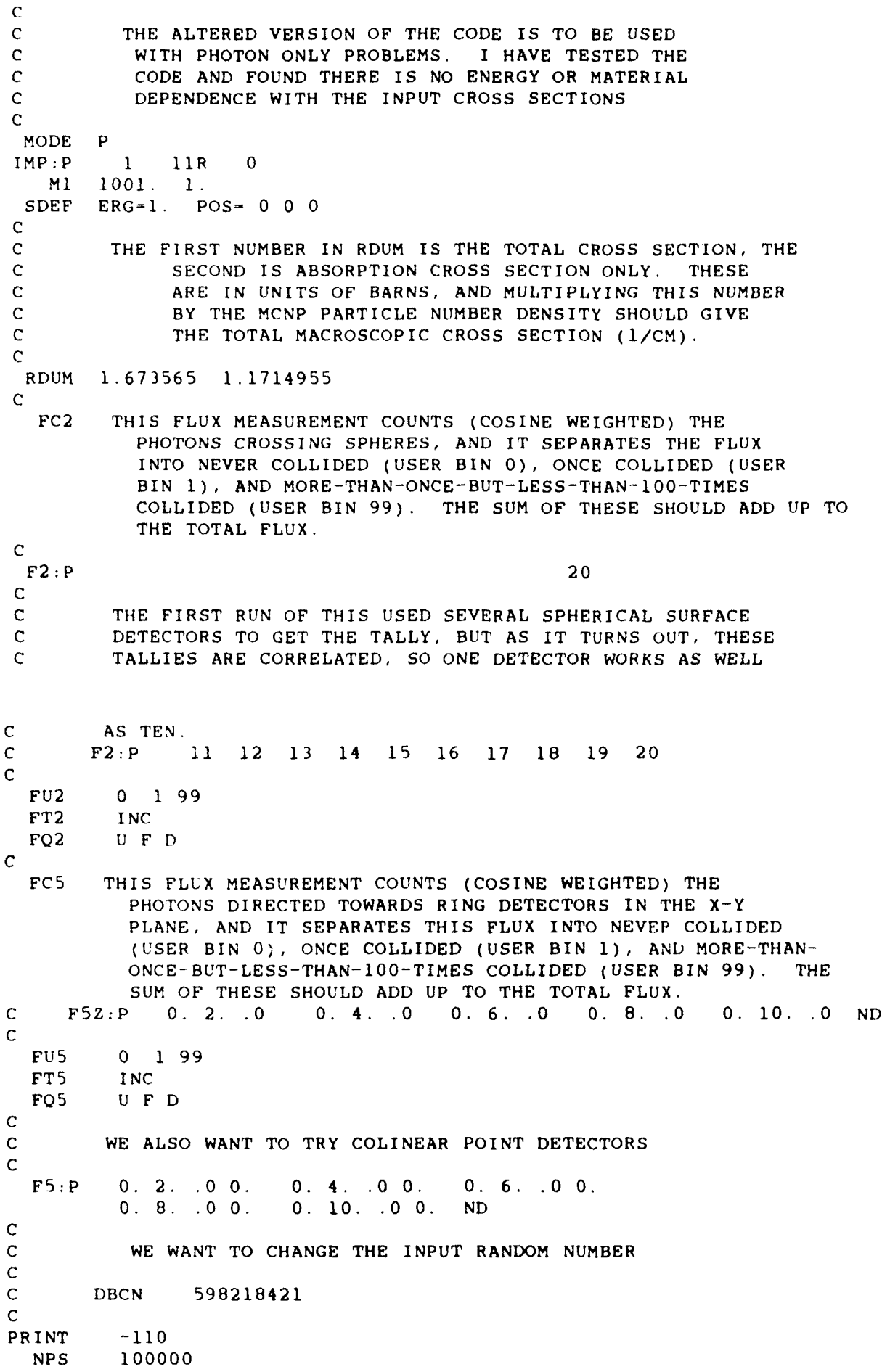

Table A.3: (cont) 


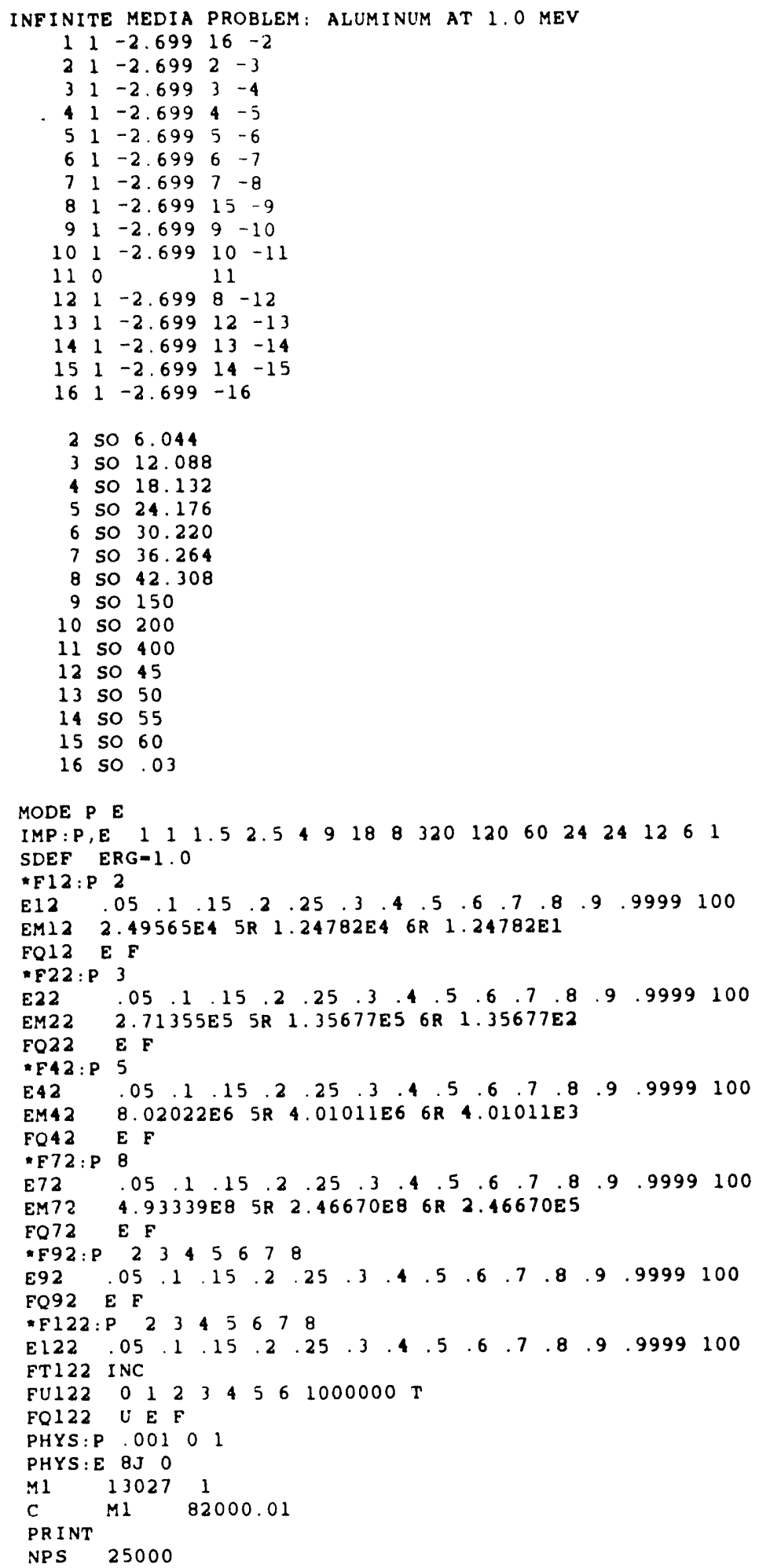

Table A.4: Input file for Benchmark Three: Infinite media problem Al at $1.0 \mathrm{McV}$. 


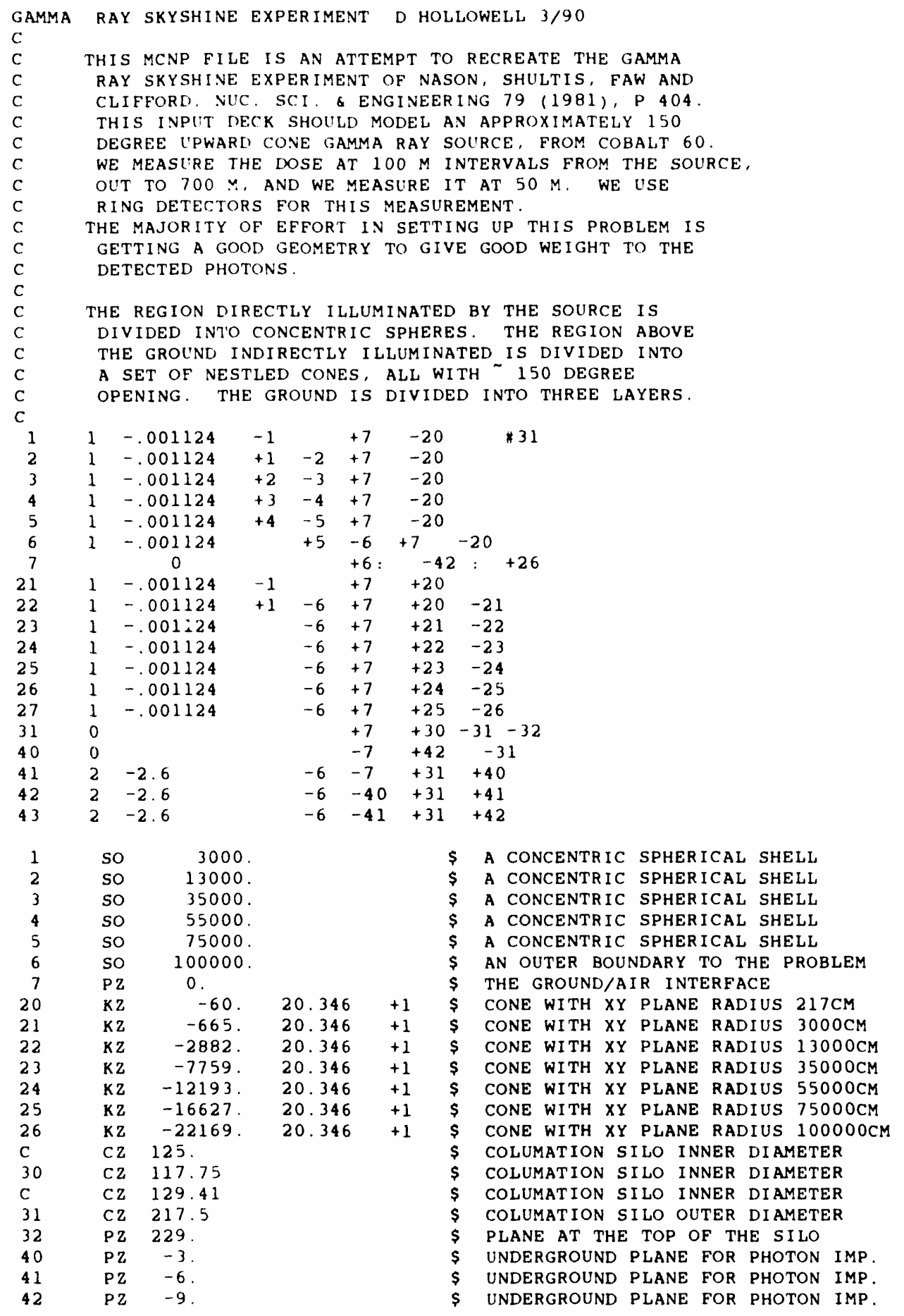

Table A.5: Input file for Benchmark Four: Gamma skyshine experiment. 
C THE IMPORTANCES HAVE BEEN FOUND, MORE OR LESS, BY TRIAL AND ERROR $\begin{array}{cccccccc}\text { IMP : P } & 1 & 1.7 & 2 & 3.3 & 6.7 & 17 . & 0 \\ & 10 . & 2.0 & 3 & 7.0 & 27 . & 100 . & 400 . \\ & 0 . & 0 . & 2 . & 4 . & 6 .\end{array}$
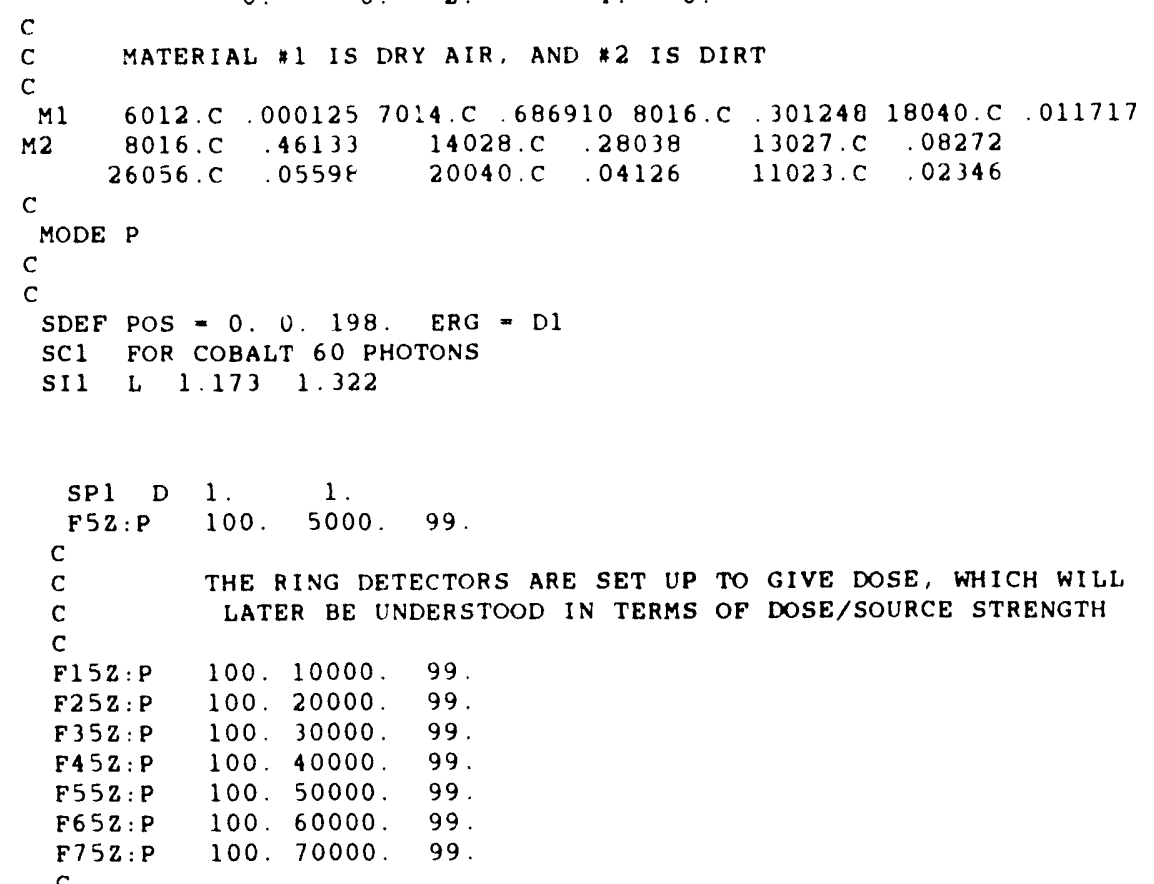

NOTE THAT MCNP WILL GIVE FLUX AS 1/CM* 2 , PER SOURCE PARTICLE. TO CHANGE THIS TO A DOSE WE USE THE FLUX MULTIPLIER CARD "FM". FOR A GIVEN * TALLY, THE FM CARD FM\# RHO M $-5-6$ "WILL PRODUCE A TALLY WITH DOSE UNITS MEV/CM"*3. IN THIS CARD "RHO" IS THE NUMBER DENSITY (\#/(CM*BARN)) OF THE ABSORBING PARTICLES AND "M" IS THE MATERIAL NUMBER OF THE ABSORBING PARTICLES. THE -5 AND -6 SPECIFY TO INCLUDE (RESPECTIVELY) THE INTERACTION CROSS SECTION AND THE HEATING FRACTION. THIS "FM" CARD PROVIDES RESULTS IN MEV/CM* 3 PER HISTORY, AND WE WANT, FOR INSTANCE, [RAD/HISTORY]. SINCE 1 RAD $=100$ ERG/G, WE CAN CONVERT THE NUMBER PRODUCED BY THIS "FM" CARD TO [RAD/HISTORY] BY MULTIPLYING THE TALLY RESULT BY

$[(1.602 \mathrm{E}-6(\mathrm{ERG} / \mathrm{MEV})] *(1(\mathrm{RAD}) / 100(\mathrm{ERG} / \mathrm{G}))] /$ [MASS DENSITY (G/CM**3)]]

WHICH IS $1.43 E-05$ IF THE DENSITY IS .0011 G/CM*3 .. THIS MULTIPLICATIVE FACTOR TAKES MEV/CM *3/HISTORY TO RAD/HISTORY. IN THE CASE OF NASON SHULTIS AND FAW, THEY WANTED AN ANSWER IN MICRORAD/HOUR/CI (WHERE CI IS SOURCE STRENGTH IN CURIES). FOR A 666 CI SOURCE. THEN, TO CONVERT FROM RAD/HISTORY TO MICRORAD/HR/CI WE MULTIPLY FURTHER, BY A FACTOR:

Table A.5: (cont) 


$\begin{array}{lllll}\text { FM5 } & 4.541 E-05 & 1 & -5 & -6 \\ \text { FM15 } & 4.541 E-05 & 1 & -5 & -6 \\ \text { FM25 } & 4.541 E-05 & 1 & -5 & -6 \\ \text { FM35 } & 4.541 \mathrm{E}-05 & 1 & -5 & -6 \\ \text { FM45 } & 4.541 \mathrm{E}-05 & 1 & -5 & -6 \\ \text { FM55 } & 4.541 \mathrm{E}-05 & 1 & -5 & -6 \\ \text { FM65 } & \mathbf{4 . 5 4 1 E - 0 5} & 1 & -5 & -6 \\ \text { FM75 } & 4.541 \mathrm{E}-05 & 1 & -5 & -6 \\ \text { C } & & \\ \text { C } & \text { THE LOW ENERGY PHOTONS ARE NOT WORTH THE BOTHER } \\ \text { C } & \text { SINCE THEY ARE BELOW THE DETECTOR RESPONSE FUNCTION CUTOFF } \\ \text { CUT :P } & 1 \text { E+33 } 0.0399 \\ \text { C } & \end{array}$

Table A.5: (cont) 


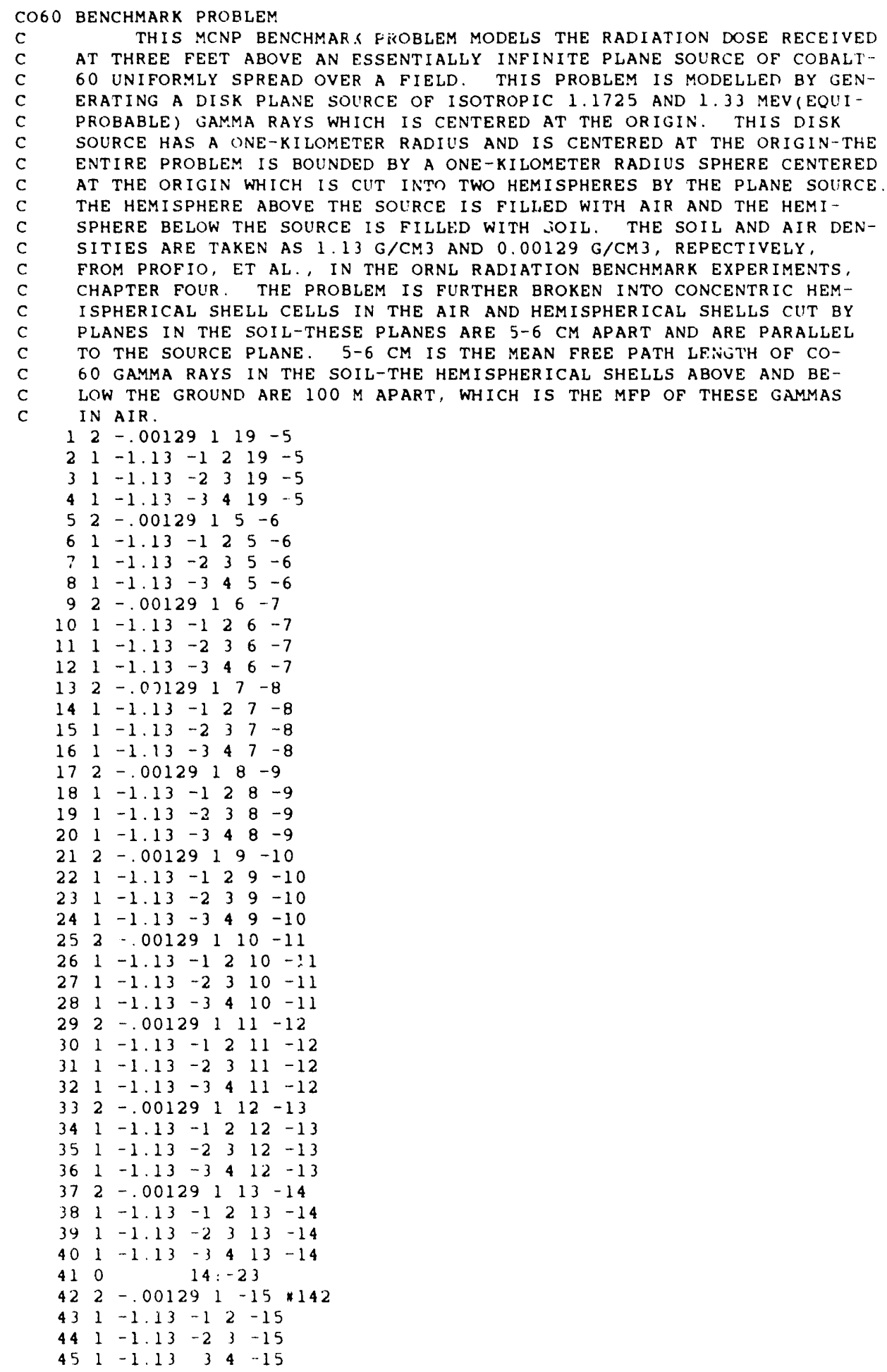

Table A.6: Input file for Benchmark Five: Air over ground. 


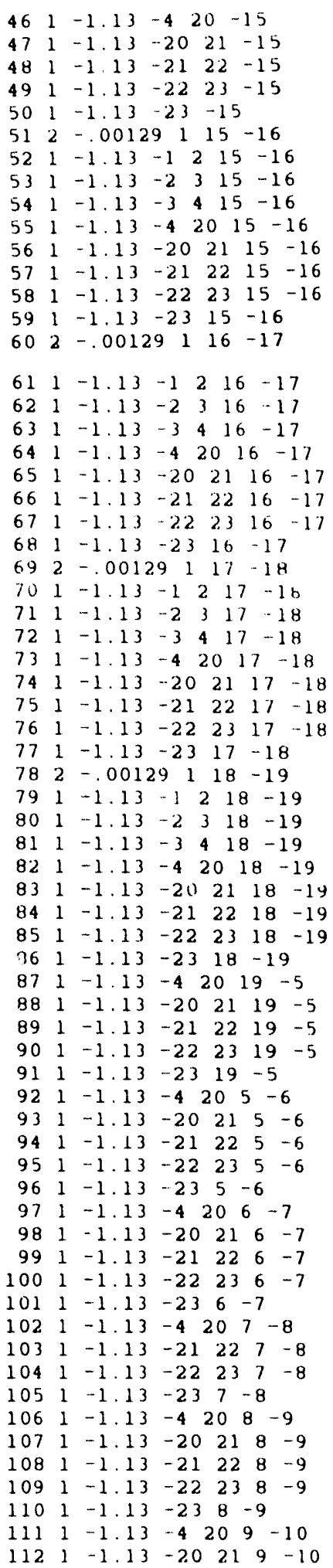

Table A.6: (cont) 


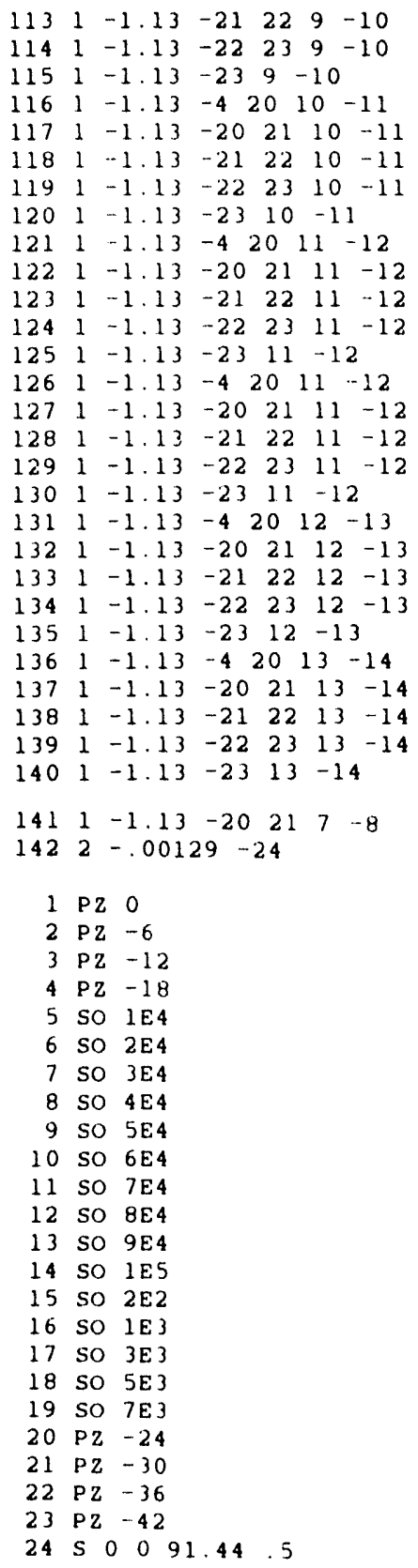

Table A.6: (cont) 


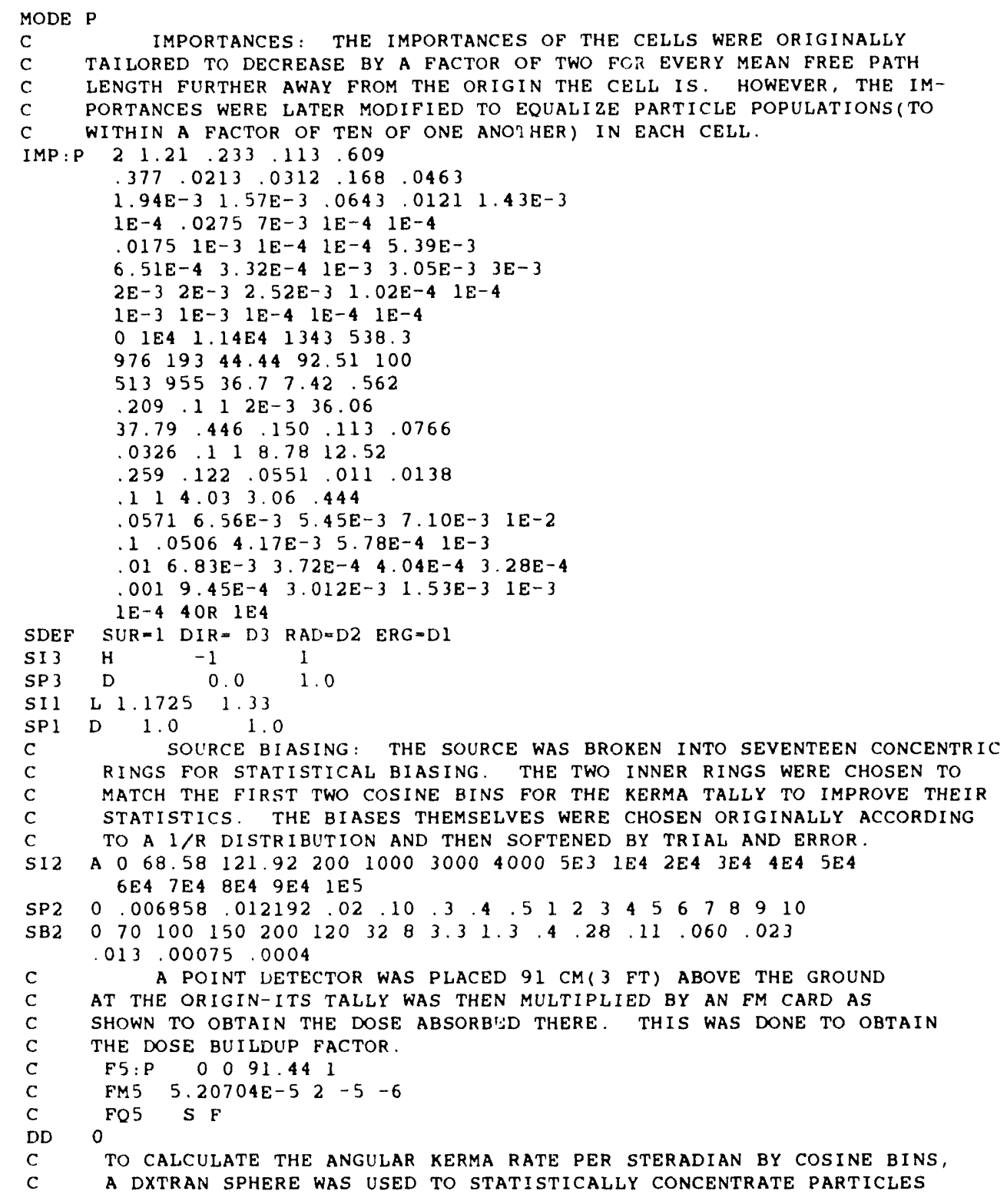

Table A.6: (cont) 


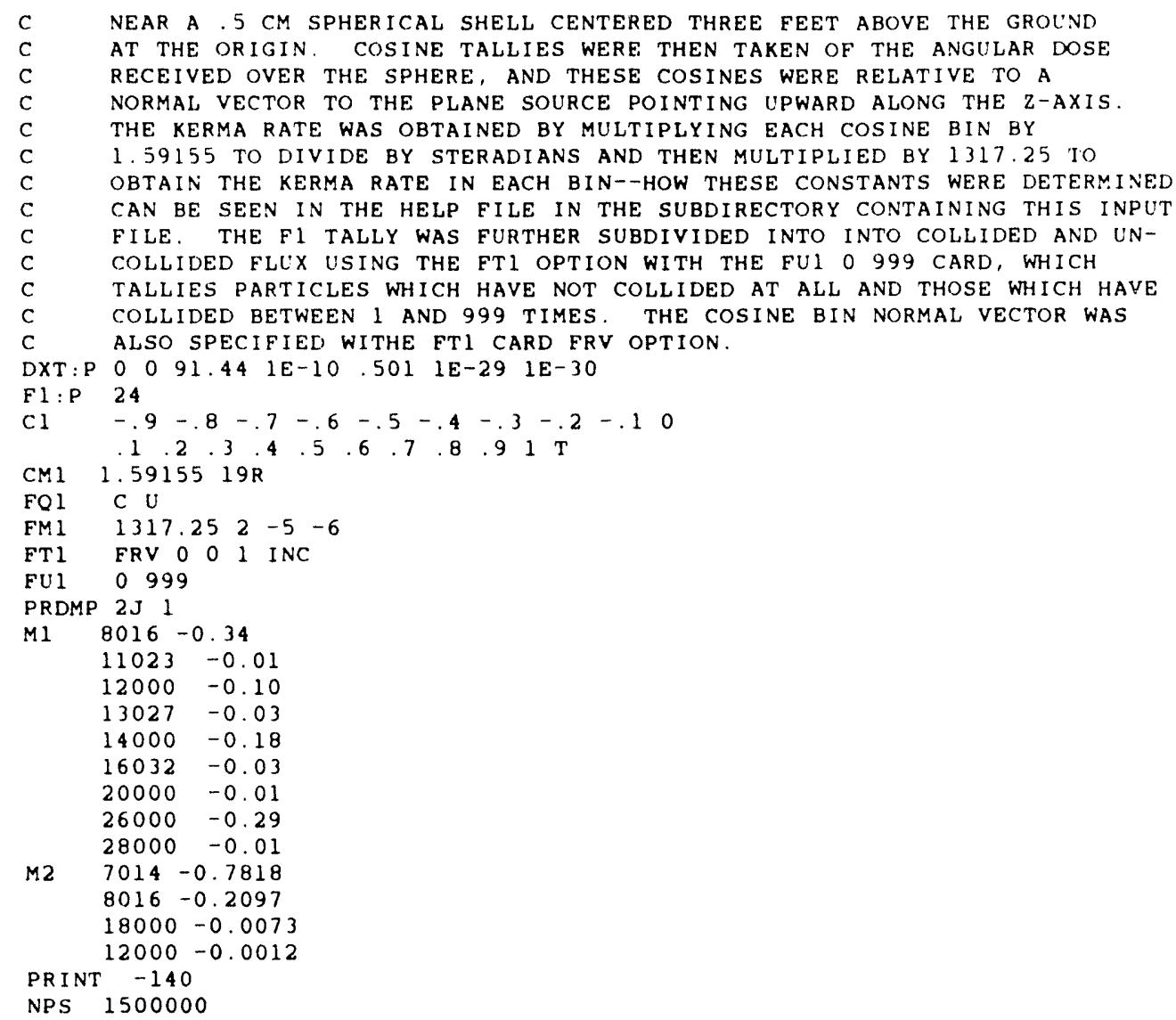

Table A.6: (cont) 


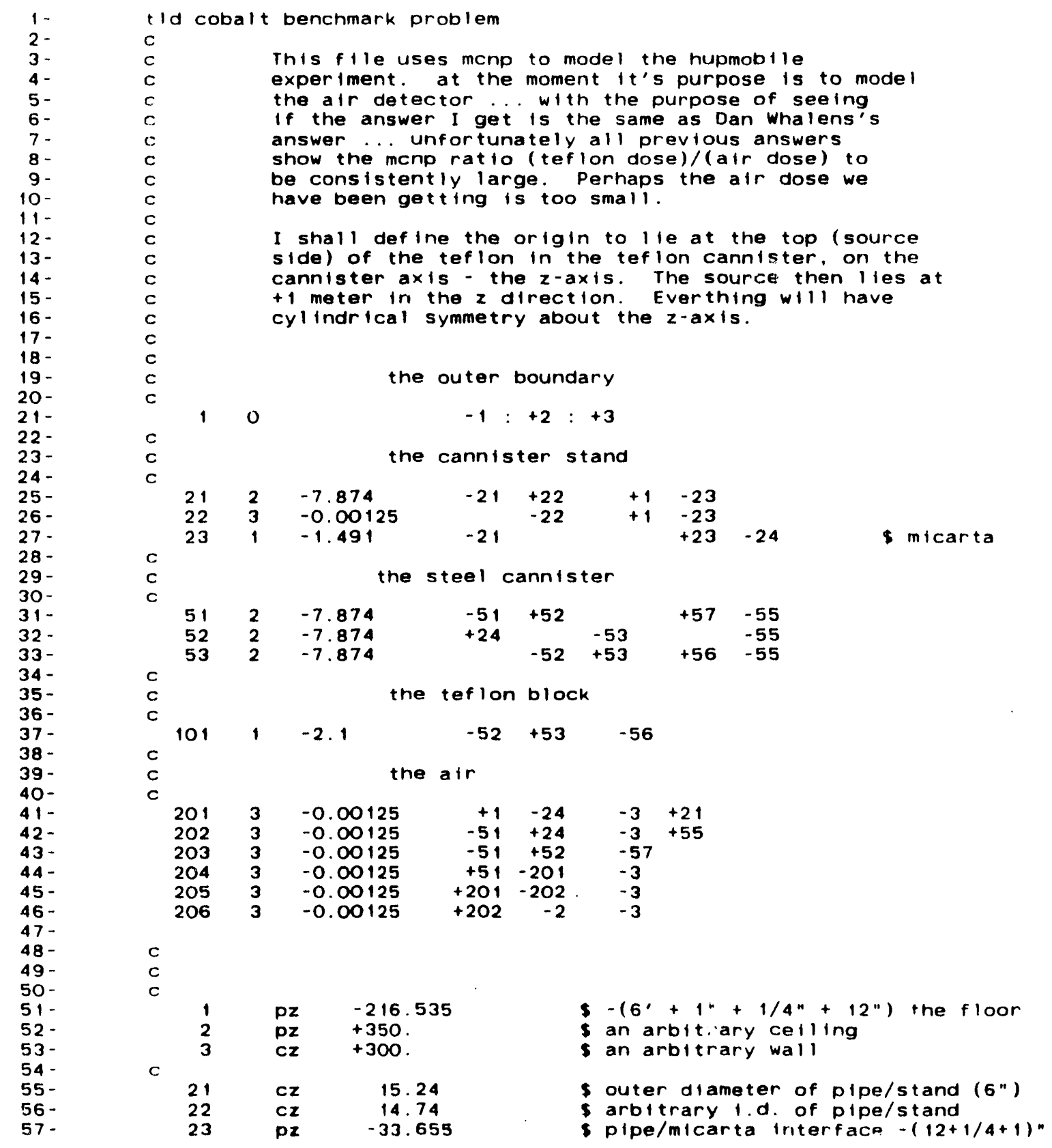

Table A.7: Input file for Benchmark Six: Hupmobile air dose geometry. 


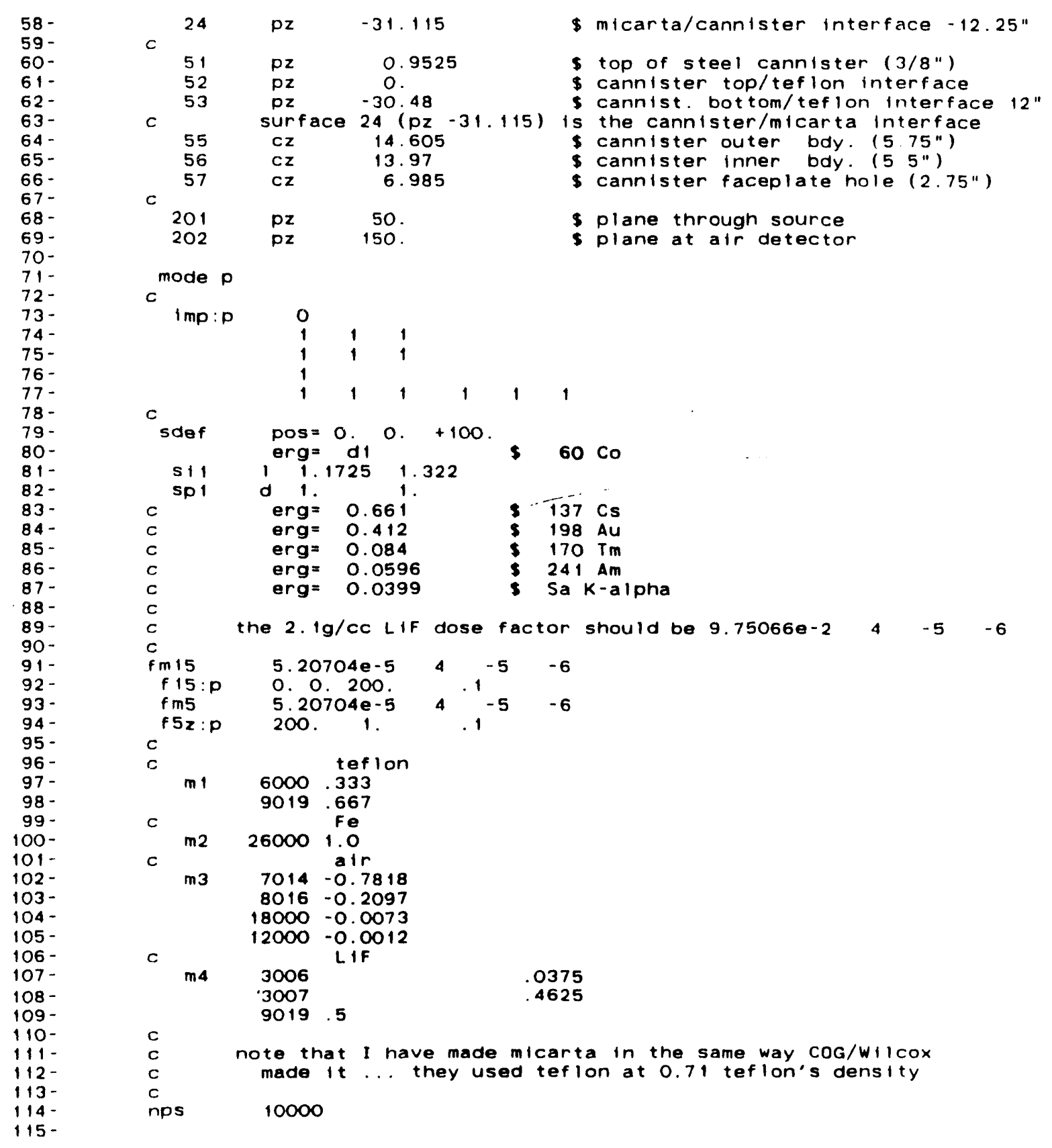

Table A.7: (cont) 


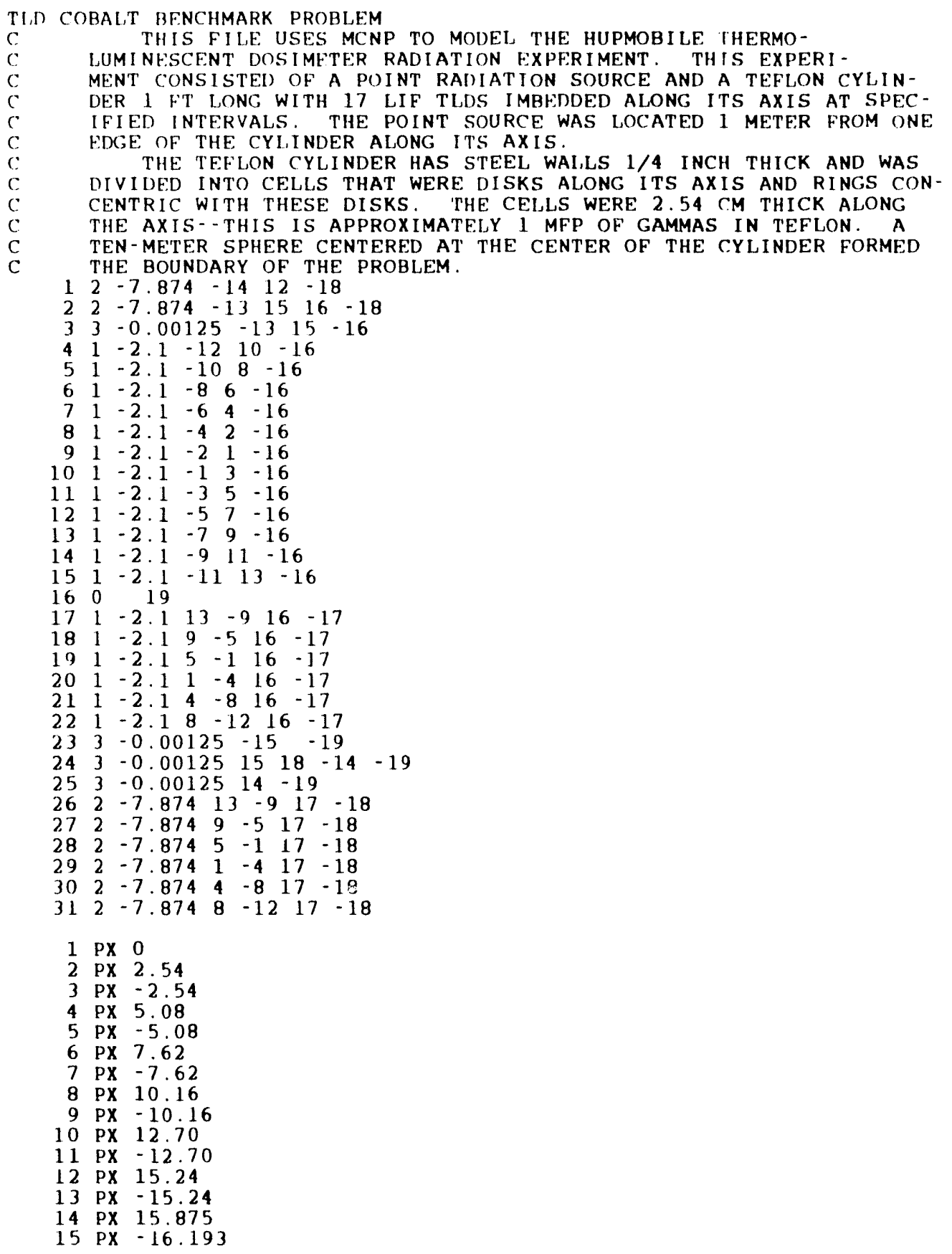

Table A.8: Input file for Benchmark Six: Hupmobile ${ }^{60}$ Co teflon dose geometry. 


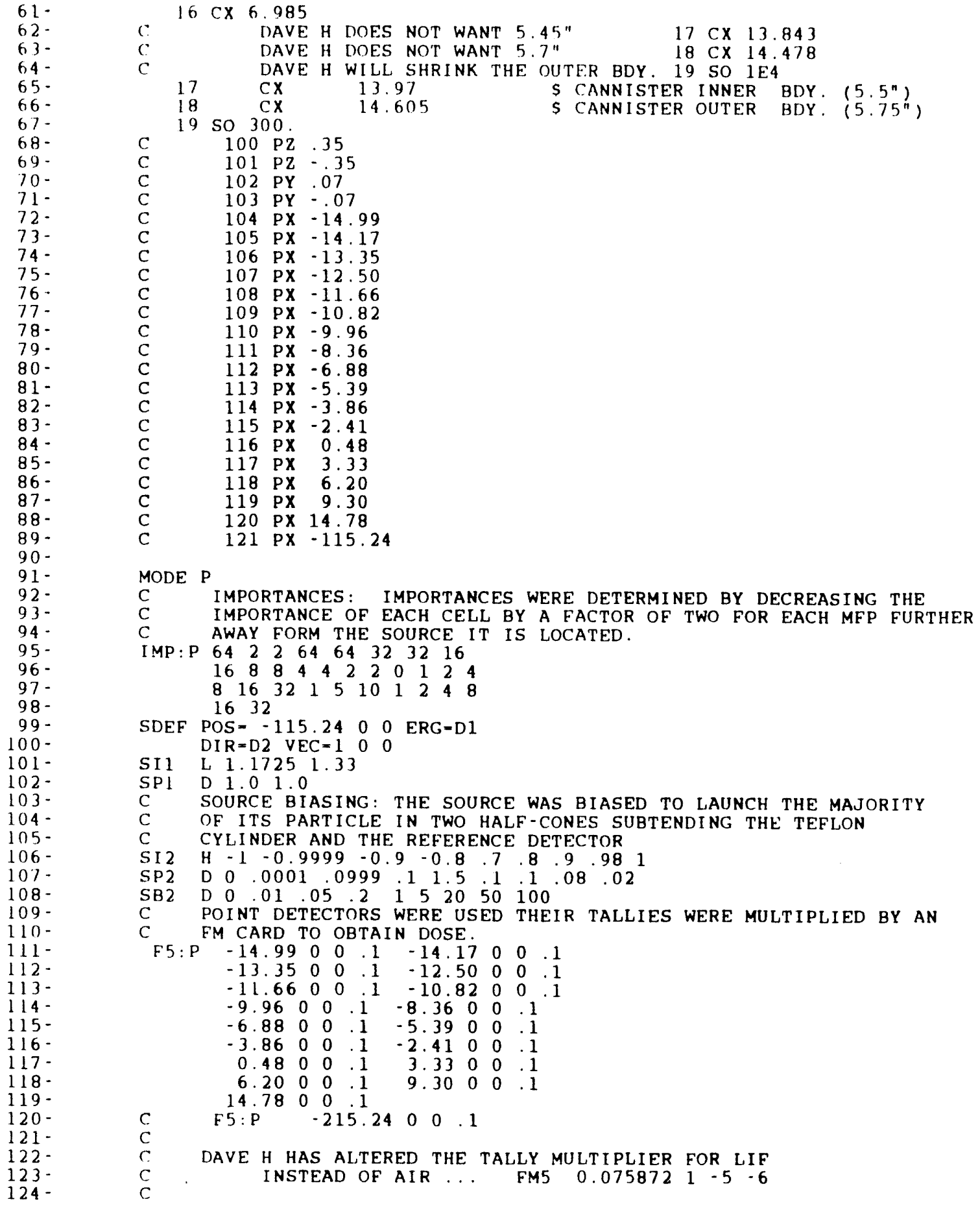

Table A.8: (cont) 
141

$142-$

$143-$

144 .

145

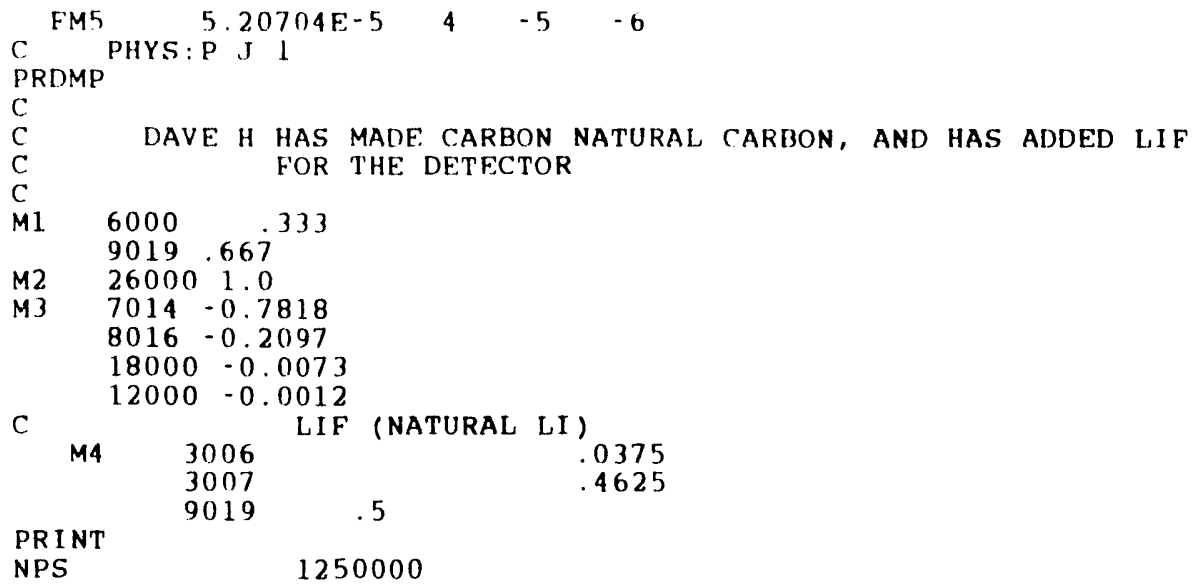

Table A.8: (cont) 


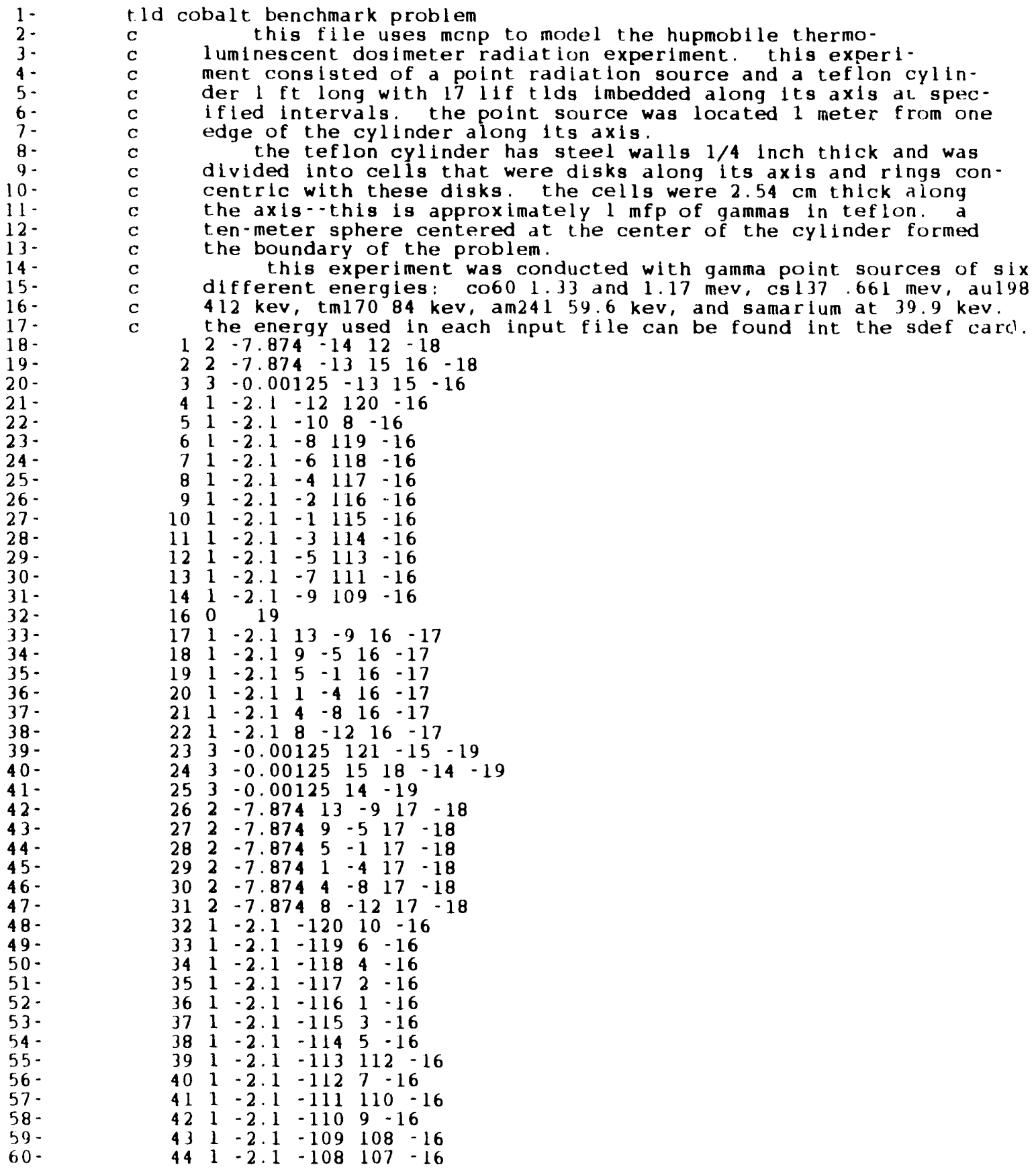

Table A.9: Input file for Benchmark Six: Hupmobile $\mathrm{Sm}_{\alpha} \mathrm{K}_{\alpha}$ teflon dose germetry: 


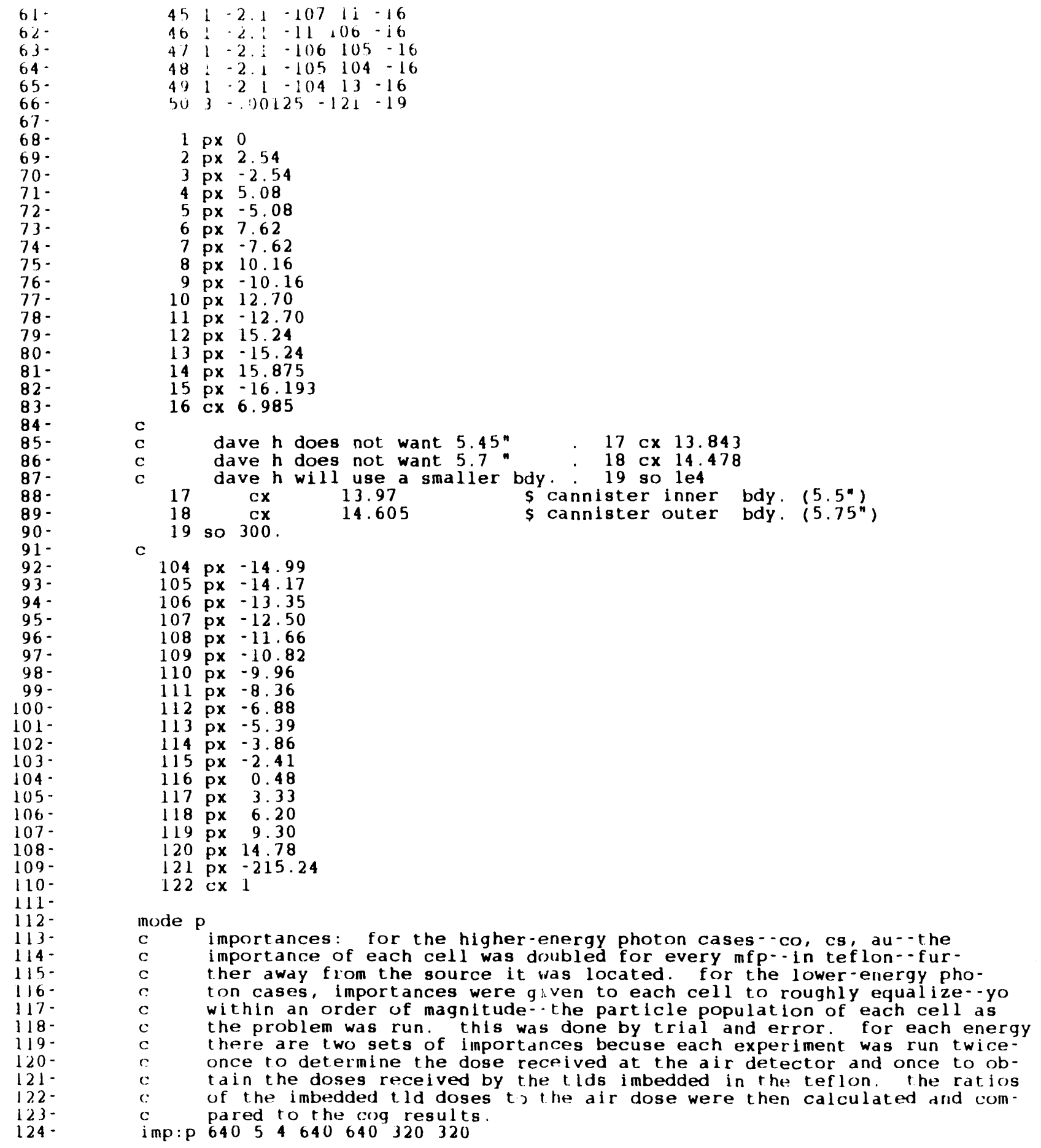

Table A.9: (cont) 


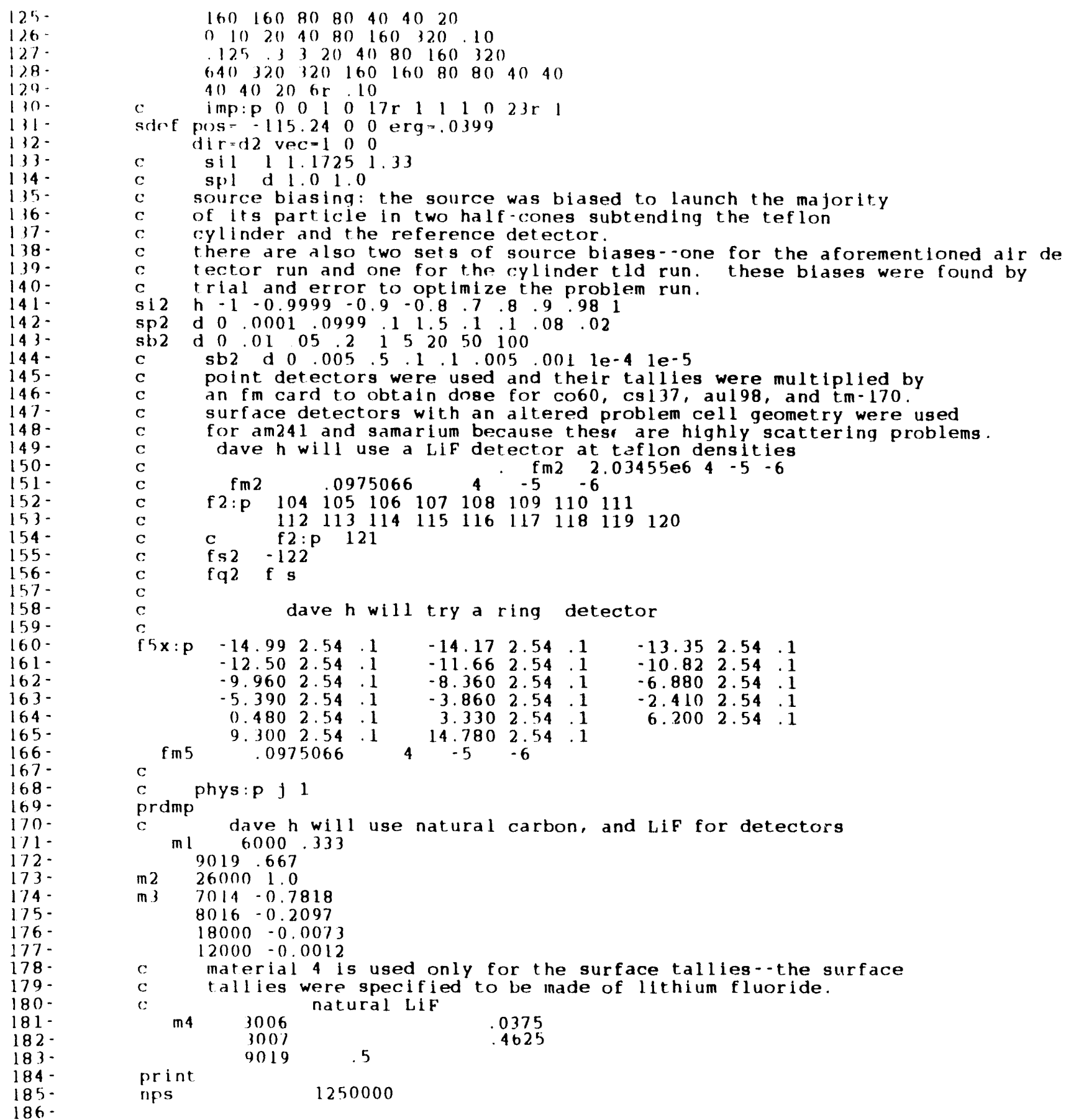

Table A.9: (cont) 

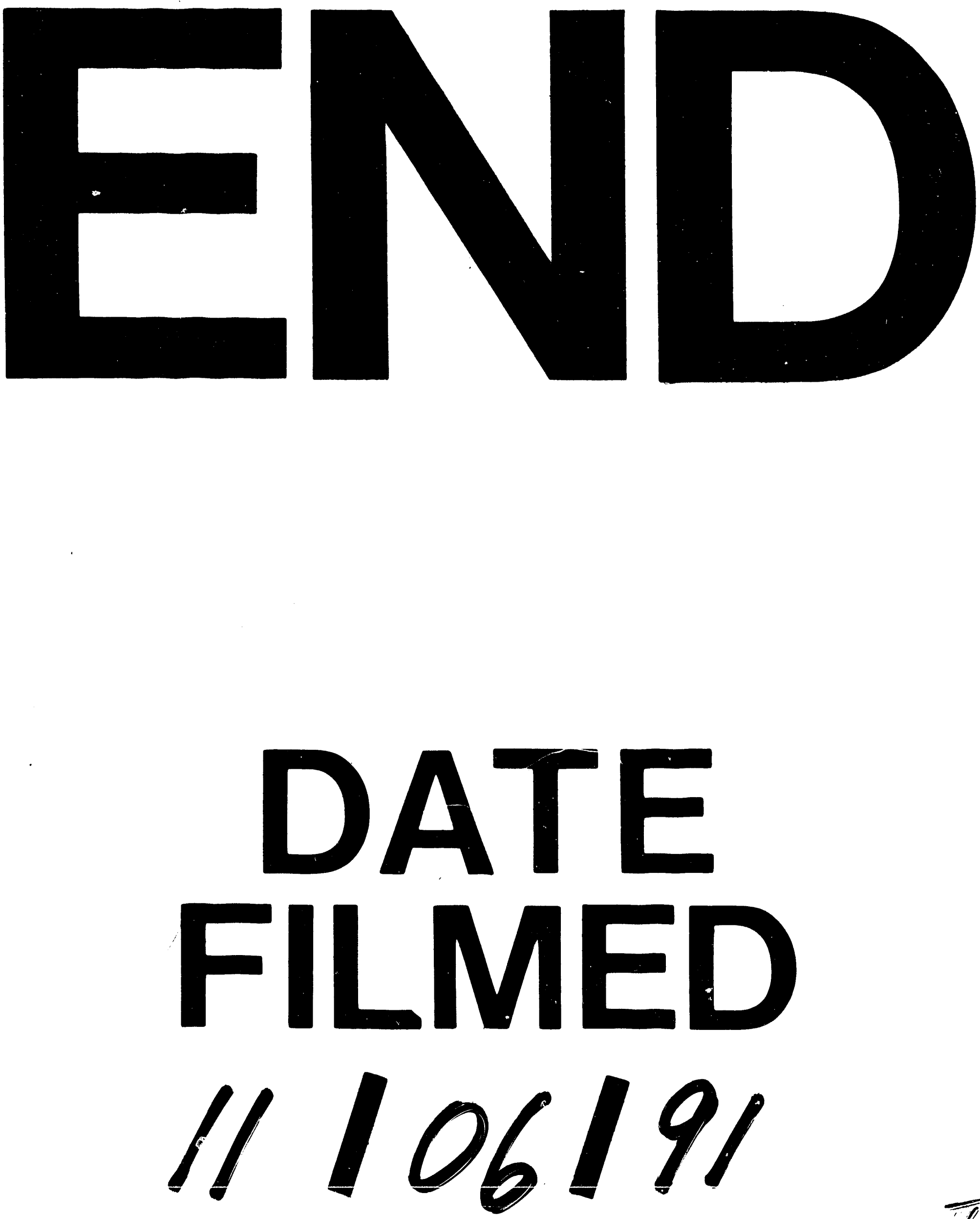

प 